\title{
A MULTIDIMENSIONAL DATA MODEL WITH SUBCATEGORIES FOR EXPRESSING AND REPAIRING SUMMARIZABILITY
}

\author{
by \\ Mohammad Ariyan
A thesis submitted to
the Faculty of Graduate Studies and Research
in partial fulfillment of
the requirements for the degree of
DOCTOR OF PHILOSOPHY \\ In Computer Science \\ at \\ CARLETON UNIVERSITY
}

Ottawa, Ontario

September, 2014

œ Copyright by Mohammad Ariyan, 2014 


\begin{abstract}
In multidimensional (MD) databases summarizability is a key property for obtaining interactive response times. With summarizable dimensions, pre-computed and materialized aggregate query results at lower levels of the dimension hierarchy can be used to correctly compute results at higher levels of the same hierarchy, improving efficiency. Being summarizability such a desirable property, we argue that established MD models cannot properly model the summarizability condition, and this is a consequence of the limited expressive power of the modeling languages. In addition, because of limitations in existing MD models, algorithms for deciding summarizability and cube view selection are not efficient or practical.

We propose an extension to the Hurtado-Meldelzon (HM) MD model, the EHM model, that includes subcategories and explore its properties specially in addressing issues related to summarizability. We investigate the extended model as a way to directly model MDDBs, with some clear advantages over HM models. Most importantly, EHM is -in a precise technical sense- more expressive than HM for modeling MDDBs that are subject to summarizability conditions. Moreover, given an MD aggregate query in an EHM database, we can determine in a practical way (that only requires processing the dimension schema as opposed to the instance), from which minimal subset of pre-computed cube views it can be correctly computed.

Our extended model allows for a repair approach that transforms non-summarizable HM dimensions into summarizable EHM dimensions. We propose and formalize a twostep process that involves modifying both the schema and the instance of a non-summarizable HM dimension.
\end{abstract}




\section{Table of Contents}

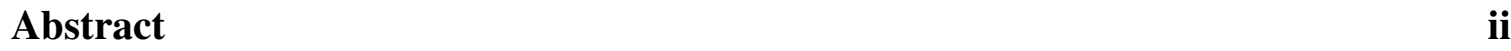

List of Figures

Acknowledgements $\quad$ viii

$\begin{array}{lll}\text { Chapter } 1 & \text { Introduction } & 1\end{array}$

$\begin{array}{lll}\text { Chapter } 2 & \text { Background and Literature Review } & 13\end{array}$

2.1 Multidimensional Databases . . . . . . . . . . . . . . . . . 13

2.2 MD Queries . . . . . . . . . . . . . . . . . . . . . . 14

2.2.1 MD Queries in SQL . . . . . . . . . . . . . . . . 15

2.2.2 The MDX Query Language . . . . . . . . . . . . . . . . . 16

2.2.3 Datalog With Aggregation . . . . . . . . . . . . . . . 17

2.3 Summarizability . . . . . . . . . . . . . . . . 18

2.4 Data Repairs of MDDBs . . . . . . . . . . . . . . . 20

2.5 Related Work: State of the Art . . . . . . . . . . . . . . . . 22

2.5.1 Multidimensional Data Models . . . . . . . . . . . . . 22

2.5 .2 Summarizability . . . . . . . . . . . . . . 24

2.5.3 Cube View Selection . . . . . . . . . . . . . . . . . . 29

$\begin{array}{lll}\text { Chapter } 3 & \text { Structural Repairs of Multidimensional Databases } & 31\end{array}$

3.1 Structural Repairs . . . . . . . . . . . . . . . . . . . . 31

3.2 Properties of Structural Repairs . . . . . . . . . . . . . . . . . . . . . . 36

3.3 Virtual Repairs in MDX . . . . . . . . . . . . . . . . . . . . . . . 40

3.4 Discussion . . . . . . . . . . . . . . . . . . . 42

$\begin{array}{lll}\text { Chapter } 4 & \text { The Extended HM Model } & 45\end{array}$

4.1 The Multidimensional Model . . . . . . . . . . . . . . . . . . . . . 45 
4.2 Creating EHM Dimensions . . . . . . . . . . . . . . . . . . . 48

4.2.1 The ADD-LINK Algorithm . . . . . . . . . . . . 51

4.3 EHM and ROLAP schemas . . . . . . . . . . . . . . . . . . 54

Chapter $5 \quad$ EHM and Summarizability 56

5.1 Flexible Summarizability for EHM . . . . . . . . . . . . . . . . . 56

5.2 Computing Summarizability Sets in HM . . . . . . . . . . . . . . . 60

5.3 Computing Summarizability Sets in EHM . . . . . . . . . . . . . . . 62

5.3.1 The COMPUTE-SUMSETS Algorithm . . . . . . . . . . . . . . . 64

5.4 EHM and Expressiveness . . . . . . . . . . . . . . . . . . 68

$\begin{array}{lll}\text { Chapter } 6 & \text { Query Answering } & 73\end{array}$

6.1 The Datalog Setting . . . . . . . . . . . . . . . . . . . 73

6.2 MD Aggregate Queries . . . . . . . . . . . . . . . . . . . . . 74

6.3 The Query Answering Problem . . . . . . . . . . . . . . . . . . . . . 77

6.4 Query answering in EHM . . . . . . . . . . . . . . . . . . . 78

Chapter $7 \quad$ Repairs of Non-Summarizable HM Dimensions $\quad 85$

7.1 The EHM-based Repair Process _ . . . . . . . . . . . . . . . . . . . . 85

7.2 Canonical Mapping: From HM to Compliant EHM Dimensions . . . . . . 86

7.2.1 The FIND-PCM Algorithm _ . . . . . . . . . . . . . . . 89

7.3 E-repairs . . . . . . . . . . . . . . . . . 95

7.4 Discussion . . . . . . . . . . . . . . . . . . . 100

$\begin{array}{lll}\text { Chapter 8 } & \text { Experiments } & 102\end{array}$

8.1 Introduction . . . . . . . . . . . . . . . . . . . . . . 102

8.2 Experimental Setting . . . . . . . . . . . . . . . . . 103

8.3 Creating HM Dimensions vs Finding Preferred Canonical Mappings . . . . 104

8.4 Query Answering Performance . . . . . . . . . . . . . . . . . . . 107

$\begin{array}{lll}\text { Chapter } 9 & \mathbf{1 1 5}\end{array}$

9.1 Conclusions . . . . . . . . . . . . . . . . . . 115 
9.2 Future Work . . . . . . . . . . . . . . . . . . . . 117

$\begin{array}{ll}\text { Bibliography } & 119\end{array}$ 


\section{List of Figures}

1.1 Sales database $\ldots \ldots \ldots \ldots \ldots 2$

1.2 The Employee dimension: employees can have multiple parents . . 4

1.3 A summarizable yet not strictly-summarizable dimension . . . . . 5

$1.4 \quad$ An EHM Location dimension . . . . . . . . . . . . . . 8

$2.1 \quad$ A minimal data repair for the Location dimension of Figure 1.1 . . 21

2.2 Data repairs of the Location dimension . . . . . . . . . . . . 27

$3.1 \quad$ An SR for the Location dimension . . . . . . . . . . . 33

3.2 Another SR for the Location dimension . . . . . . . . . . . . . 33

3.3 Another minimal SR for the Location dimension . . . . . . . . 36

$3.4 \quad$ Structural repairs and non-strictness f . . . . . . . . . . . 43

$3.5 \quad$ Combining structural repairs and data repairs . . . . . . . . . . . . 44

4.1 Steps of the add link algorithm for creating the Location dimension 48

4.2 Star implementation of the Location EHM dimension . . . . . . 55

$5.1 \quad$ An HM dimension with non-transitive summarizability sets . . . . 61

$6.1 \quad$ The Location and Date EHM dimensions . . . . . . . . . . 75

7.1 Data vs EHM-based repairs (complexities are only in terms of instance size $) \ldots \ldots \ldots \ldots \ldots$. . . . . . . . . . . . . 86

7.2 A non-summarizable result of a canonical mapping for the Location dimension . . . . . . . . . . . . . . . . 87

7.3 A minimal e-repair for the compliant EHM instance of Figure 7.2 . . 96

8.1 Instance size vs dimension creation time (for small instances) . . . . 105

8.2 Instance size vs dimension creation time (for large instances) . . . . 106

8.3 The effect of adding categories on creation time . . . . . . . . 108

8.4 HM schemas for dimensions Location, Date, Product and Employee 109 
8.5 Query answering time for selected queries . . . . . . . . 113

8.6 The impact of fact table size on query answering time . . . . . . . . 114 


\section{Acknowledgements}

First, I offer my sincerest gratitude to my supervisor, Professor Leopoldo Bertossi, for his guidance throughout this research. He has supported and encouraged me with his knowledge and patience. He has a been a true professional. The work presented in this thesis would not have been possible without his advice and support.

I would like to thank members of my $\mathrm{PhD}$ proposal and thesis committees Dr. Illuju Kiringa and Dr. Douglas Howe, and members of my comprehensive examination committee, Dr. Carlisle Adams and Dr. Jean-Pierre Corriveau.

I dedicate this thesis to my mother. Making you happy and proud has always been my motivation. You have always supported me and never asked for anything in return. From the deepest part of my heart, I love you and I will always miss you. 


\section{Chapter 1}

\section{Introduction}

On-Line Analytical Processing (OLAP) was a notion introduced by Codd [24] to characterize a set of requirements for consolidating, viewing and analyzing data organized according to the multidimensional data model (MDM). In recent years, OLAP has become one of the central issues in database research, and OLAP technologies have become important applications in the database industry.

A multidimensional database (MDDB) is an implementation of a multidimensional data model. It combines data from different sources, for analysis and decision support. MDDBs represent data in terms of dimensions and fact tables. Dimensions themselves are usually graphs, and their structure defines the levels of detail at which data can be summarized for visualization and manipulation.

In an MDM, a dimension is modeled by two hierarchies: a dimension schema and a dimension instance. A dimension schema is a hierarchy composed of a number of levels or categories, each of which represents a different level of granularity. Instantiating the dimension schema by values at each level forms the dimension instance and creates another hierarchy parallel to the category hierarchy. A row in the fact table corresponds to a measurement that is taken at the intersection of the categories at the bottom of the dimensions: a quantity that is given context through base dimension values.

This MD organization allows users to aggregate data at different levels of granularity, corresponding to different levels of categories in the hierarchies. Aggregation is the most important operation in OLAP applications. OLAP queries are complex, may involve massive data, and also aggregation. Therefore, fast query answering is a crucial task in OLAP [46].

Some models of MDDBs have been proposed in the literature [3, 17, 31, 45, 47, 59, $60,71]$, ranging from simple cube models that view data as n-dimensional cubes, to hierarchical models that explicitly capture the structural relationship between categories in 


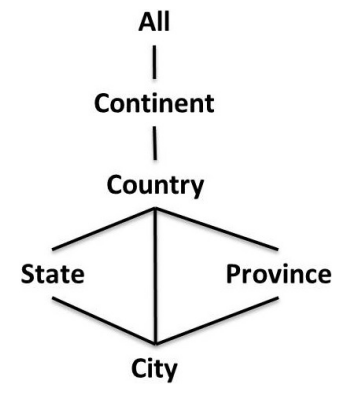

(a) Location schema

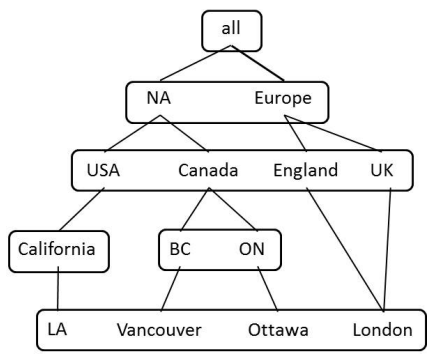

(b) Location instance

\begin{tabular}{|c|c|c|}
\hline City & Date & Sales \\
\hline Ottawa & Jul 1,14 & 6000 \\
Vancouver & Jul 1,14 & 4500 \\
LA & Jul 1,14 & 10000 \\
Ottawa & Jul 2,14 & 5500 \\
Vancouver & Jul 3,14 & 1400 \\
Ottawa & Jul 3,14 & 3000 \\
\hline
\end{tabular}

(c) Fact table

Figure 1.1: Sales database

the dimensions. We have adopted as basis for our research the Hurtado-Mendelzon (HM) model of MDDBs [37, 41]. It is an established and extensively investigated model, and it provides solid and clear foundations for further investigation on MDDBs.

Example 1 We represent data about a company sales for different times and locations in Figure 1.1. We illustrate there only the Location dimension, represented as a hierarchy of places where the company has branches. The Location dimension schema is in Figure 1.1a, and a possible instance for it is shown in Figure 1.1b.

In this schema, City, State, etc., are categories. Country is a parent of categories State and Province; and an ancestor of category City. Category City is a base category, i.e. it has no children. At the instance level, element LA of City rolls up to USA, as an ancestor element in category Country. LA and Vancouver are base elements. As we can see, both the schema and the instance are endowed each with compatible partial orders (lattices) that are directed from the bottom to the top.

Figure 1.1c shows the fact table containing data about sales for cities (of the base category) at different times. Here, Sales is the single measure attribute of the fact table.

The hierarchical structure of a dimension schema allows us to derive the results of aggregate queries posed on non-base (i.e. higher level) categories directly from the fact table. However, due to the possibly very large volumes of data in MDDBs, computation from scratch of aggregate queries should be avoided whenever possible. Ideally, aggregate query results at lower levels (i.e. more detailed, pre-computed views of data) should be used to correctly calculate results at higher levels of the hierarchy (i.e. less detailed views 
of data). As an example, for the instance in Figure 1.1b, it is always possible to compute sales values for different continents directly from the fact table. However, if possible, it is more practical to derive the results by aggregating over pre-computed sales values for one (or a combination) of categories from a level higher than City (in this case one of categories Country, State or Province or a combination of these categories). For this derivation to happen correctly, every base element that rolls up to some element in the parent category must pass through one and only one element from the ancestor categories [41]. If this is the case for all higher level categories, we say that the dimension instance is summarizable.

Summarizability can be defined as the ability of an aggregate query to correctly compute a cube view from another cube view where both cube views are defined in a single dimension. This notion can be applied to an instance in local terms: if a parent category and a set of its descendant categories allow for this correct derivation, we say that the parent is (locally) summarizable from those descendants. As an example, category All is summarizable from Continent, but Country is not summarizable from Province: the base elements LA and London do not pass through any element from Province when rolling up to Country. Also, category Continent is not summarizable from Country because element London rolls up to element Europe from more than one path (once through England and once through UK). In the absence of summarizability, a multidimensional database will either return incorrect query results when using pre-computed views, or lose efficiency by having to compute the answers from scratch $[42,53]$.

It is worth noting that in our Location dimension example, the issue of multiple parents could have been avoided in the ETL phase of data warehouse design. However, in some cases we actually need multiple parents (or ancestors) for an element in a single upper level dimension. The Employee dimension of Figure 1.2 is such an example. Some employees (shown in red) are supervised by more than one manager. Therefore, having multiple parents should be allowed and cannot be avoided even in the ETL phase.

Global summarizability (i.e. summarizability of a dimension) can be defined in one of two ways: relative to all the descendants of a category or only to a subclass of them. Accordingly, we say that a dimension is strictly-summarizable if every category is (locally) summarizable from each one of its descendant categories. This is the concept that has been 


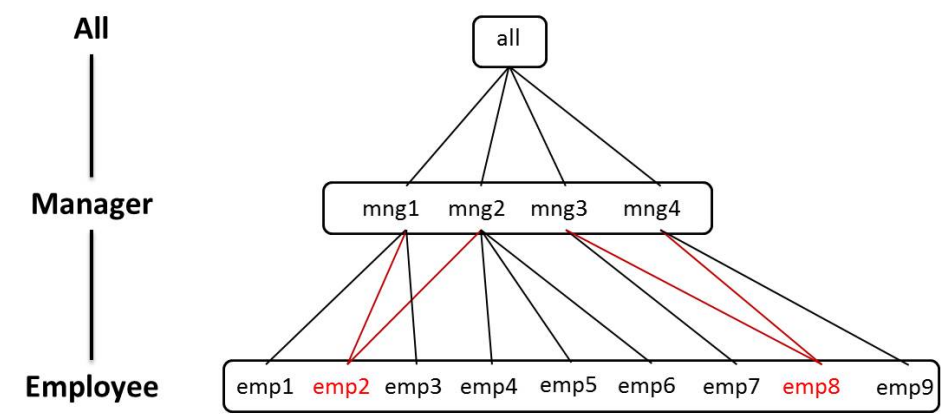

Figure 1.2: The Employee dimension: employees can have multiple parents

the focus of previous research in the literature $[13,15,20,21,41,72]$. In particular, it has been established that for a dimension to be strictly-summarizable, it must satisfy three conditions $[42,72]$ :

1. The dimension schema must have a single base category, which contains all the elements that have no children.

2. The dimension instance must be strict, i.e. each element in a category must have at most one parent in each upper category.

3. The instance has to be homogeneous (aka. covering), i.e. each element in a category has at least one parent element in each parent category.

The last two conditions can be seen as semantic constraints on a dimension instance. Dimensions that do not satisfy strictness or homogeneity (or both) are said to be inconsistent. The Location dimension instance in Figure 1.1 has a single base category, City, but it is inconsistent. The instance violates strictness: London rolls up to the two different elements in Country: England and UK. It is also non-homogeneous, i.e. heterogeneous: London does not roll up to any element in State or Province. Dimensions may become inconsistent for several reasons, in particular, as the result of a poor design or a series of update operations [44].

Notice above that for dimensions with multiple base categories, homogeneity and strictness does not necessarily guaranty summarizability. Dimensions with multiple base categories can be useful when/if a single column of the fact table needs to refer to elements of multiple different categories (e.g. a case where employees and managers can both sell products). However this is not very common in practice. 

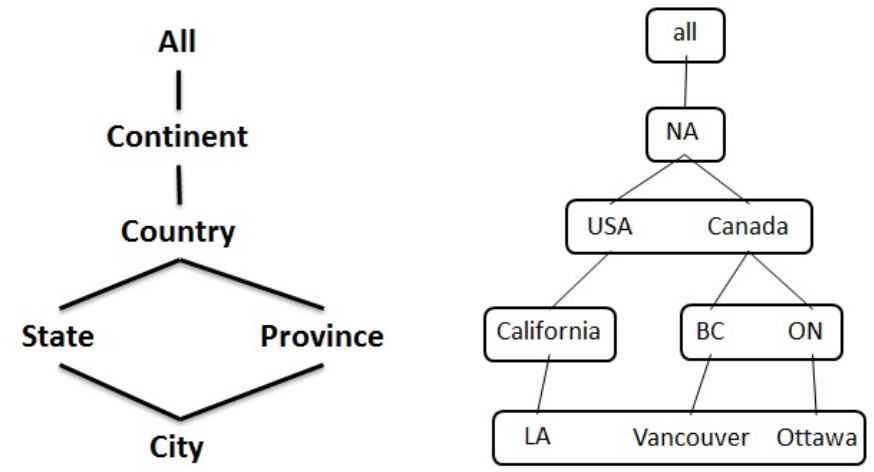

Figure 1.3: A summarizable yet not strictly-summarizable dimension

We refer to the combination of these three conditions as the strict-summarizability conditions. We argue that the strict-summarizability conditions are too restrictive, and imposing them will filter out a wide range of useful models and instances. This includes any dimension that has multiple base categories. However, there are other cases. As another example, the instance in Figure 1.3 shows that aggregate results for category Country cannot be derived from State, nor from Province. Therefore, the dimension is not strictlysummarizable. However, Country is summarizable from the combination of State and Province. Therefore, we can still take advantage of pre-computed cube views at the level of State and Province to compute aggregate results for Country. We will redefine the notion of summarizability for a dimension instance to take multi-child aggregation into consideration.

The restrictions imposed by strict-summarizability are not the whole problem. It is not only that an instance at hand fails to be summarizable. Actually, there are many commonly used dimensions for which the HM metamodel (i.e. the framework that supports the creation of specific HM models) fails to accommodate an MD model, including instances, that satisfies summarizability. This is because any HM model that captures the external reality will be bound, already by the sheer MD schema used, to have instances that do not satisfy the summarizability conditions expressed above. An example is given by Figure 1.3: when capturing in an HM model a real-world assumption, such as having State and Province, at the same level, as immediate and partitioning ancestors of City, there is no HM model that can capture this domain in a summarizable way. Every reasonable instance (e.g. with non-empty categories) will not be summarizable. 
One way to approach the problem of modeling such domains consists in making changes to the dimension schema and/or the dimension instance to restore strictness and homogeneity. In accordance with the area of repairs and consistent query answering (CQA) in relational databases $[7,11,12]$, a resulting dimension instance could be called a repair of the original one. A minimal repair is one that minimally differs (in some prescribed sense of minimality) from the original dimension and satisfies strictness and homogeneity $[9,13,72]$. As expected, minimality can be defined and characterized in different ways [11].

Previous work on repairing MDDBs has focused mainly on changing the dimension instance by modifying data elements or links between them [13, 15, 20, 19, 72]. Repairs obtained in this way are called data repairs. These repairs have been proposed to deal mainly with non-strictness, and usually assuming homogeneity $[13,19]$. When both non-strictness and heterogeneity are addressed [15, 20, 72], the latter has been confronted through the insertion of null values $[40,65,72]$. There exist real world domains, such as the one in Example 1, where this approach may produce an unnatural solution, e.g. for the instance in Figure 1.1b), one that connects London to California in State, and to BC or a null in Province. The process leads to an unnatural repair, that does not quite conform to the external reality. Of course, additional restrictions could be imposed on the repair process, like not allowing such links, but in some cases there might be no repair then.

In some cases, inconsistency may be caused by a problematic dimension schema. Therefore, changing the dimension instance may be only a temporary solution. Future update operations will probably produce new inconsistencies. So, exploring and considering structural changes, i.e. changes in the schema, is a natural way to go. This is an idea that has been suggested in [40], to overcome heterogeneity. In [9], we introduced and investigated the notion of structural repair, as an alternative to data repair. They modify the schema of an inconsistent dimension, to restore strictness and homogeneity.

Structural repairs restrict changes to dimension instances by allowing changes that affect only the category lattice and the distribution of the data elements in different categories. In particular, there are no "data changes" (as in data repairs), in the sense that the data elements and also the edges between them remain. In the case of a ROLAP data warehouse 
(with relational schemas, like star or snow flake) fewer data changes on a dimension instance directly results in fewer changes on records in the underlying database.

With data repairs, a single change on a dimension instance directly results in several changes on records in the underlying relational database (the number of changes depends on the underlying ROLAP schema, and is usually larger when changes are made at higher levels of the instance [72]). With structural repairs this is not the case, which is advantageous for users who do not want their records changed. On the other hand, with structural repairs, some sort of query reformulation becomes necessary since old queries may refer to categories of the old schema that may have changed or disappeared (with consequent changes on the relational schemas).

In this thesis, as an alternative to repairs for confronting the modeling problem mentioned above, we introduce and investigate an extension of the HM metamodel (Section 4.1). This extension allows us to produce extended HM (EHM) models. The main ingredient of this extension is the addition of subcategories. Figure 1.4 shows an EHM model of the Location dimension. Subcategories are shown as rectangles inside a category (when a subcategory coincides with its containing category, we omit the inner rectangle). The idea, that we develop in this work, is that, by introducing subcategories Country1 and Country2, category Continent now becomes summarizable from its direct lower level, in this case from subcategory Country1. We did not have summarizability in any HM model of this dimension.

We investigate the extended model as a way to directly model MDDBs, with some clear advantages over HM models. Most importantly, EHM is -in a precise technical sense- more expressive than HM for modeling MDDBs subject to summarizability conditions. In particular, it follows that a summarizable HM model is still a summarizable EHM model, without any changes. Also, there are common real-world examples for which no summarizable HM model exists, but can be modeled using a summarizable EHM model.

Compared to arbitrary HM models, EHM models have a positive computational feature: Given a category, one can decide whether it is (locally) summarizable from an arbitrary subset of its descendants without having to process the dimension instance (which is usually very large). Furthermore, this decision algorithm can be extended in order to compute the class of all subsets of descendants from which the category is summarizable. EHM models 

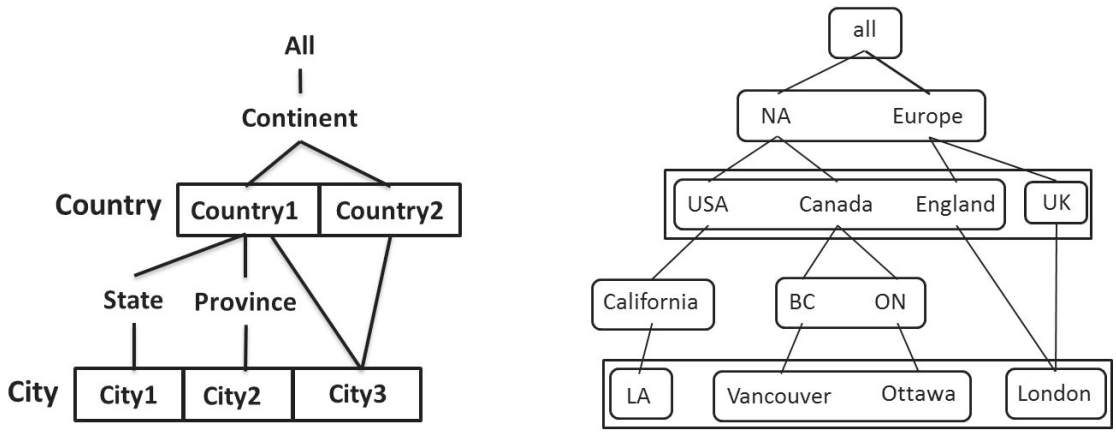

Figure 1.4: An EHM Location dimension

enjoy this feature because subcategories reflect the exact structure of the dimension instance (but at a much higher level or granularity, which reduces size). As expected, this behavior comes at the expense of imposing additional semantic constraints on dimension instances. This will have an impact on the population and maintenance of the dimension instance. However, this cost is more than offset by the benefits for OLAP query answering. More precisely, when confronted with an aggregate query $\mathcal{R}$, it will be possible to determine (at a preprocessing step) the most appropriate set of pre-computed aggregate views at lower levels that can be used to efficiently answer $\mathcal{R}$.

Notice that, for an arbitrary, non-extended HM model, a decision procedure and an algorithm for computing "summarizable classes of children" as for EHM models will also depend on the dimension instance, which is usually very large. Therefore, a similar preprocessing step becomes impractical for aggregate query answering in HM models. It should be noted that although the EHM metamodel is an extension of the HM metamodel, not necessarily an HM dimension (including the schema and instance) is an EHM dimension, because the latter are subject to additional conditions, namely roll-up relations between pairs of subcategories must be total functions. As a consequence, the algorithms for the EHM model just described do not necessarily apply to HM models, as expected.

The semantic conditions on subcategories of EHM dimension instances (i.e. the condition of roll-up relations being total functions) have to be enforced and maintained. We provide an algorithm for populating a EHM dimension instance. It is based on first specifying the categories, and then adding data links one by one. In some cases that seem to be rare in practice, this may require updating the subcategory schema. More precisely, splitting a subcategory in two (within the same category), and creating a new link to the upper 
subcategories. Actually, this approach opens the possibility of creating EHM models from scratch which we also investigate in Section 4.2.

We show that the extended HM model does not produce complications with the implementation of MDDBs as/on relational databases (ROLAP) [48]. Actually, our extended HM models can be captured by the well-known and familiar ROLAP schemas, like star or snowflake, with minor modifications.

We address query answering in a multidimensional setting with EHM models. Intuitively, the query answering problem is, given an MD database, an aggregate query and a set of pre-computed cube views, to find another query (i.e. a rewriting) that produces the same results using the pre-computed cube views.

The subcategory structure of a set of EHM dimensions provides an important feature which is to specialize a cube view to selected subcategories. This feature allows us to derive the result of an aggregate query by combining a subset of cube views that are specialized to selected subcategories. We show that finding such a selection of specialized cube views can be done efficiently for EHM dimensions (without processing dimension instances).

The EHM model allows for a repair approach that transforms non-summarizable HM dimensions into summarizable EHM dimensions. We propose a two-step process that involves modifying both the schema and the instance of an inconsistent HM dimension.

First, by the addition of subcategories, an inconsistent HM instance is transformed into an EHM instance through a process called canonical mapping (CM). A CM result contains the same set of categories, and the same elements and links in the dimension instance, but adds new subcategories and links between them in the schema. A preferred canonical mapping (PCM) is one that minimizes the redistribution of elements in new subcategories. We will show that such minimization helps avoid potential future joins within queries for recombining elements of a category, thus improving query answering performance.

We provide an algorithm that, given an inconsistent HM instance, produces one of the possible PCMs. In theory, exponentially many PCMs may exist for an HM instance. Our algorithm chooses only one of those candidates in polynomial time with regards to the HM instance size. We can decide in polynomial time whether a compliant EHM instance is a PCM of an inconsistent HM instance. In most cases, the PCM that is the result of the first step is summarizable. If this is not the case, another set of (instance-based) changes need 
to be applied to enforce summarizability.

In the second step of the repair process, changes are applied to links in the dimension instance of a chosen PCM to enforce summarizability, resulting in a so-called e-repair. As before, we tend to minimize link changes within the dimension instance, the result being a minimal e-repair. We will show that all minimal e-repairs of a PCM (or more generally, of a compliant EHM instance) can be found without the need to process the dimension instance.

Comparing the EHM-based repair process described above with the usual data repair approach presented in $[13,15,20,19]$, the former incurs in lower computational cost for restoring summarizability of an inconsistent HM instance. In [20], it is shown that the problem of computing a minimal data repair is NP-hard(as opposed to the EHM-repair process which is P-TIME) with regard to instance size. In addition, we show that the result of the EHM-based repair process is more natural compared to the data and structural repair approaches.

The main focus of our research has been on creating summarizable dimensions either from scratch or by modifying existing non-summarizable HM models. Consequently, we can take advantage of the benefits of summarizability with regards to query answering. The contributions of this dissertation can be summarized as follows:

1. We explore and investigate structural repairs as an alternative to data repairs. They modify the schema of an inconsistent dimension, to restore strictness and homogeneity. We establish that under certain conditions, a structural repair reduces the cost wrt changing the dimension instance. We also show how query-scoped calculated members in MDX can be used to create virtual repairs that simulate structural repairs. Investigating the properties of this schema-based repair approach becomes a basis for the development of our extended model (Chapter 3).

2. We introduce and formalize the Extended HM models (EHM models) for MDDBs. Categories and links are still the user-defined ingredients of an EHM schema. However, subcategories are an additional ingredient that, if structured properly within categories of an EHM schema, enforce summarizability and provide the subsequent benefits. We also specify when an HM model can be considered as a particular kind of EHM model (Chapter 4). 
3. We show how EHM dimension instances can be populated from scratch (Section 4.2). We propose the ADD-LINK algorithm that takes categories (but not subcategories) as user-defined inputs. Data elements can then be added one by one opening the possibility of incrementally creating EHM models from scratch.

4. We show that EHM models can be implemented with the usual ROLAP schemas, with minor modifications. Additional tables will be added corresponding to categories that contain more than one subcategory (Section 4.3).

5. We propose a new, less restrictive notion of summarizability that applies to both HM and EHM dimensions (Section 5.1). We show that: (a) Summarizable HM models are still summarizable as EHM models. (b) There are sensible real-world examples for which no summarizable HM model exists, but that can be modeled using a summarizable EHM model, showing a difference in expressive power, between the HM and EHM models.

6. We propose the COMPUTE-SUMSETS algorithm to determine from which precomputed cube views a cube view (i.e. aggregate query) can be correctly computed. We show that this problem can be solved for EHM models with less computational cost compared to arbitrary HM models (Section 5.3).

7. We provide a formal characterization of expressiveness for classes of dimension models. We establish that the class of summarizable HM dimensions is less expressive than that of summarizable EHM dimensions (Section 5.4).

8. We formalize the problem of query answering using pre-computed cube views in an MD setting. We show that, given a set of materialized pre-computed cube views in an EHM setting, we can effectively rewrite new queries in terms of available views and obtain query answers with better performance (Chapter 6).

9. We introduce and investigate an EHM-based repair approach that transforms nonsummarizable HM dimensions into summarizable EHM dimensions. We provide the FIND-PCM algorithm that, given an arbitrary HM instance, generates an EHM instance with the same set of categories and the same set of elements and links in the dimension instance. We also show how summarizability can be guarantied by 
modifying links in the dimension instance. We also analyze the complexity of this approach and compare it to that of the traditional data repair approach on HM instances (Chapter 7).

10. Finally, we show results of our experiments. Specifically, we have investigated, through experiments, how much time it takes to create an MD database comprised of EHM dimensions in comparison with that of a database containing HM dimensions. We have experimented with a wide range of dimension sizes as well as different fact table sizes. In addition, our experiments will show how query answering performance is affected by the introduction of subcategories in EHM dimensions (Chapter 8).

It is worth noting that in the life cycle of a data warehouse, the extended model will appear in the design phase. However, the changes will not be manual, in the sense that the users will not need to provide additional information to create and maintain subcategories. Instead, the subcategory structure can be maintained automatically using our creation algorithms. Of course, the performance improvements of these design changes will appear in the query answering phase. 


\section{Chapter 2}

\section{Background and Literature Review}

\subsection{Multidimensional Databases}

A multidimensional database (MDDB) is a data repository that provides an integrated environment for decision support queries that require complex aggregations on huge amounts of data [10]. A model representing an MDDB is composed of logical cubes, facts and dimensions.

A data cube is a data structure that allows fast analysis of data. It can also be defined as the capability of manipulating and analyzing data from multiple perspectives. The arrangement of data into cubes in MDMs overcomes some limitations of relational databases. A data cube consists of numeric facts called measures which are categorized by dimensions.

Facts are measures assigned to instances of dimensions. They are typically numeric and additive. Measures populate cells of a logical cube with the facts collected about business operations.

In order to measure the facts at different granularity levels, a dimension is usually represented as a hierarchy of categories. The hierarchical structure allows the user to study facts at different levels of detail. They provide structured labeling information to, otherwise unordered, numeric measures.

Various MD models have been proposed and investigated in the literature [27, 30, 31, 48, 3, 18, 28, 43, 47, 51, 59, 66, 71]. Each model imposes different structural and semantic conditions on the hierarchy of categories. In the simplest case, the hierarchy has a linear form, i.e. every category is connected to at most one parent category and at most one child category. More advanced models represent the hierarchy of categories using complex graph structures such as lattices. We adopt as basis for our research the Hurtado-Mendelzon (HM) model of MDDBs [37, 41] (for an overview of other MD models see Section 2.5.1).

In the HM model, a dimension schema $\mathfrak{S}$ is a tuple of the form $\rangle U, \mathcal{D}, \Rightarrow \mid$, where $U$ is a possibly infinite set (the underlying data domain), Dis a finite set of categories (or better, 
category names), $\rangle \mathcal{D}, \Rightarrow \mid$ is a directed acyclic graph. Notice that $\Rightarrow$ is a binary relation between categories. Its transitive and reflexive closure is denoted by $\Rightarrow^{*}$.

Every dimension schema has a distinguished top category, All, which is reachable from every other category: For every category $c \triangle \mathcal{D}, c \Rightarrow^{*} A l l$ holds. Categories that have no descendants are called base categories.

Given a dimension schema $\mathfrak{S}$, a dimension instance for the schema $\mathfrak{S}$ is a tuple $\mathcal{H}=$ $>\mathcal{Q}, \delta,<\mid$, where $\mathcal{Q}$ is the finite subset of $U, \delta: M \nearrow \mathcal{D}$ is a total function (assigning data elements to categories), and $<$ is a binary relation on $\mathcal{Q}$ that parallels relation $\Rightarrow$ on categories: $e_{1}<e_{2}$ iff $\delta\left(e_{1}\right) \Rightarrow \delta\left(e_{2}\right)$. Element all $\triangle \mathcal{Q}$ is the only element of category All.

The transitive and reflexive closure of $<$ is denoted with $<^{*}$, and can be used to define the roll-up relations for any pair of categories $c_{i}, c_{j}$ :

$$
\left.\mathcal{S}_{c_{i}}^{c_{j}}(\mathcal{H})=\right\}\left(e_{i}, e_{j}\right) \backslash e_{i} \triangle c_{i}, e_{j} \triangle c_{j} \text {, and } e_{i}<^{*} e_{j}\langle\text {. }
$$

A fact table consists of a granularity and a measure. Measures are formalized by a set of variables. Each variable has its specific domain of values. A base fact table (usually for simplicity referred to as fact table) is the one, in which all of the categories in the granularity are base categories of their corresponding dimension schema.

\subsection{Queries}

The most common aggregate queries in DWHs are those that perform grouping by the values of a set of categories from different dimension schemas (known as a granularity), and return a single aggregate value per group. These aggregate queries are known as cube views [38]. The aggregation is achieved by upward navigation from base categories through a path in the dimension schema, which is captured by roll-up relations.

Cube views are defined using distributive aggregate functions [32], with SUM, MAX and MIN being the most common cases. We will use $\Psi_{f}\left[c_{1}, \ldots, c_{n}\right]$ to denote a cube view at granularity $\} c_{1}, \ldots, c_{n}\left\langle\right.$ where $f$ is the distributive aggregate function, and each $c_{i}$ is a category (name) from a dimension schema $\mathfrak{S}_{i}$. When we concentrate on a single dimension, the cube view will be of the form $\Psi_{f}[c]$, with $c$ a category of a dimension schema $\mathfrak{S}$.

Lets assume $\mathcal{H}_{1}, \ldots, \mathcal{H}_{n}$ are a set of $\mathrm{HM}$ dimensions with schemas $\mathfrak{S}_{1}, \ldots, \mathfrak{S}_{n}$ and base categories $b_{1}, \ldots, b_{n}$, respectively. Cube view $\Psi_{f}\left[c_{1}, \ldots, c_{n}\right]$, where $c_{k}$ is a category from 
schema $\mathfrak{S}_{k}$, can be defined as follows:

$$
\prod_{c_{1}, \ldots, c_{n}, f(m)}\left(\mathcal{S}_{b_{1}}^{c_{1}}\left(\mathcal{H}_{1}\right) \bowtie \ldots \bowtie \mathcal{S}_{b_{n}}^{c_{n}}\left(\mathcal{H}_{n}\right) \bowtie F\right)
$$

where $m$ is a measure from fact table $F$.

Example 2 For an MD schema comprised of Location and Date dimensions, cube view $\Psi_{S U M}[$ State, Year $]$ is defined as follows:

$$
\Pi_{\text {State }, \text { Year }, S U M(\text { Sales })}\left(\mathcal{S}_{\text {City }}^{\text {State }}(\text { Loc }) \bowtie \mathcal{S}_{\text {Day }}^{\text {Year }}\left(\mathcal{H}_{n}\right) \bowtie F\right)
$$

where fact table $F$ associates measure Sales to cities by day.

\subsubsection{Queries in SQL}

MD queries can be expressed using SQL aggregate queries of the following form:

SELECT $c_{j}, \ldots, \mathrm{c}_{n}, \mathrm{f}(\mathrm{a}) \mathrm{EROM} \mathrm{T}, \mathrm{R}_{i}, \ldots, \mathrm{R}_{m}$

WHERE conditions GROUP BY $\mathrm{C}_{j}, \ldots, \mathrm{C}_{n}$

Here $\mathrm{C}_{j}, \ldots, \mathrm{C}_{n}$ are attributes (categories) of the roll-up relations $\mathrm{R}_{i}, \ldots, \mathrm{R}_{m}$ (treated as tables), and $f$ is one of min, max, count, sum, avg, applied to fact measure a.

This form of aggregation using SQL assumes a relational representation of the database in which MDDBs are usually represented as a star or snowflake database [48], although other relational representations have also been investigated [72].

Example 3 Assuming we have the following star representation of the Sales database (with the dimension table of Location on the left, the fact table on the right and skipping the representation of the dimension table for Date):

(LocationStar)

\begin{tabular}{|c|c|c|c|c|c|}
\hline City & State & Province & Country & Continent & All \\
\hline LA & California & null & US & NA & all \\
\hline Vancouver & null & BC & Canada & NA & all \\
\hline Ottawa & null & ON & Canada & NA & all \\
\hline London & null & null & England & Europe & all \\
\hline London & null & null & UK & Europe & all \\
\hline
\end{tabular}

(FactTable)

\begin{tabular}{|c|c|c|}
\hline City & Date & Sales \\
\hline Ottawa & Jan 1,14 & 6000 \\
\hline Vancouver & Jan 1,14 & 4500 \\
\hline LA & Jan 1,14 & 10000 \\
\hline Ottawa & Jan 2,14 & 5500 \\
\hline Vancouver & Jan 3,14 & 1400 \\
\hline Ottawa & Jan 3,14 & 3000 \\
\hline
\end{tabular}

The following SQL query returns total sales for different countries: 


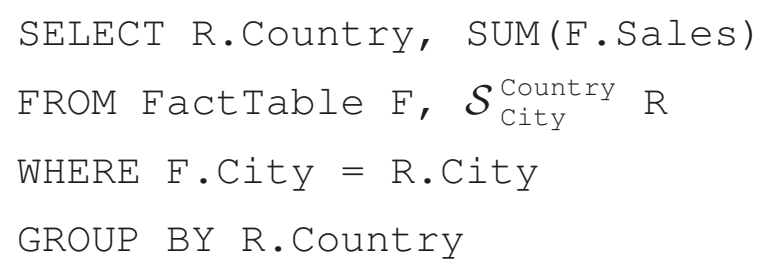

Notice that in the case of star schema, $\mathcal{S}_{\text {City }}^{\text {Country }}$ can simply be replaced by LocationStar in the query. however, in the case of snowflake schema, it should be computed (as an inner query) with a join operation over dimension tables.

\subsubsection{The MDX Query Language}

MultiDimensional Expressions (MDX) [61, 69] is a declarative query language for multidimensional databases designed by Microsoft. Results of MDX queries come back to client programs as data structures that need to be further processed to look like a spreadsheet. This is similar to how SQL works with relational databases.

Example 4 (example 3 continued) The following MDX query returns total sales for different countries:

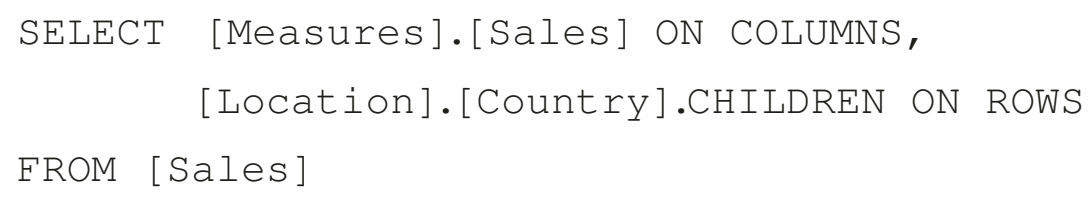

Here, Sales represents the base cube view (i.e. fact table).

An MDX query consists of axis specifications and a cube specification. It may also contain an optional slicer specification that is used to define the slice of the cube to be viewed. A simple MDX statement has the following form:

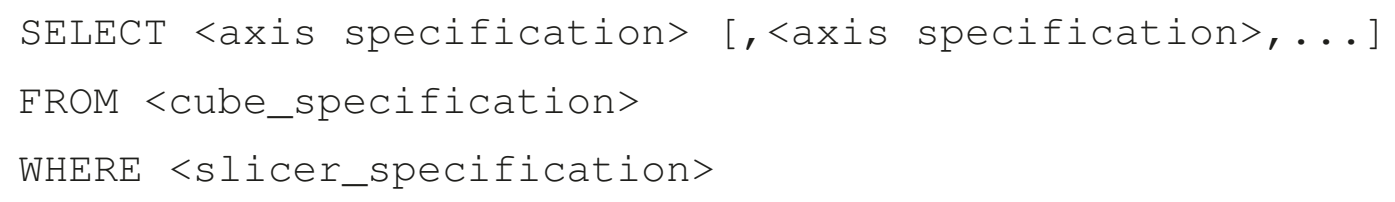

We can break this query down into pieces: 
1. The SELECT clause defines the axes for the MDX query structure by identifying data elements to include on each axis. MDX has several ways to do this. The basic possibilities are to identify data elements either by choosing members of a category or by specifying their parent element.

2. The FROM clause names the cube from which the data is being queried. This is similar to the FROM clause of SQL that specifies the tables from which data is being queried

3. The WHERE clause provides a place to specify members for other cube dimensions that do not appear in the axes and filters data based on the conditions that are provided. The use of WHERE is optional. It is used when measures are not queried in axis specifications (For simplicity, in the remaining sections we will skip the WHERE clause and include measures in our axis specifications).

Example 5 We can write a simple MDX query that returns dollar and unit sales for quarters of 2014 as follows:

\begin{tabular}{|c|c|c|c|}
\hline SELECT $\}$ [Measures].[Dollar Sales], & & Dollar Sales & Unit Sales \\
\hline [Measures].[Unit Sales]< & Q1,2014 & $1,213,380.0$ & 2,725 \\
\hline ON COLUMNS, & Q2,2014 & $855,600.0$ & 2,100 \\
\hline$\}$ [Time].[2014].CHILDREN \langle & Q3,2014 & $1,160,419.0$ & 2,518 \\
\hline ON ROWS & Q4,2014 & $1,638,560.0$ & 3,122 \\
\hline
\end{tabular}

FROM [Sales]

\subsubsection{Datalog With Aggregation}

Datalog is a declarative logic programming language that is often used as a query language for deductive databases. It has a simple uniform syntax to express relational queries and to extend relational calculus with recursion. Datalog's simplicity in expressing complex queries has made it a viable choice for the formal representation of problems related to data management and query processing.

A datalog rule is an expression of the form $R_{1}\left(u_{1}\right) \rightarrow R_{2}\left(u_{2}\right), \ldots, R_{n}\left(u_{n}\right)$ where $n \gg 1, R_{1}, \ldots, R_{n}$ are relation names and $u_{1}, \ldots, u_{n}$ are free tuples of appropriate 
arities. Each variable occurring in $u_{1}$ must occur in at least one of $u_{2}, \ldots, u_{n}$. A datalog program is a finite set of datalog rules [1].

A datalog program defines the relations that occur in heads of rules based on other relations. The definition is recursive, so defined relations can also occur in bodies of rules. Thus a datalog program is interpreted as a mapping from instances over the relations occurring in the bodies only, to instances over the relations occurring in the heads [1].

Different extensions of Datalog have been proposed to support aggregate rules $[2,4$, 25, 29]. In [25], aggregate rules take the form $P(\bar{a}, \bar{x}, \operatorname{Agg}(\bar{u})) \rightarrow B(\bar{y})$, where $P$ is the answer collecting predicate, the body $B(\bar{y})$ represents a conjunction of literals all of whose variables are among those in $\bar{y}, \bar{a}$ is a list of constants, $\bar{x} \cap \bar{u} \leq \bar{y}$, and Agg is an aggregate operator such as Count or Sum. The variables $\bar{x}$ are the group-by variables. That is, for each fixed value $\bar{b}$ for $\bar{x}$, aggregation is performed over all tuples that make $B \frac{\bar{x}}{b}$, the instantiation of $B$ on $\bar{b}$ for $\bar{x}$, true. Count $(\bar{u})$ counts the number of distinct values of $\bar{u}$, while $\operatorname{Sum}(\bar{u})$ sums over all $\bar{u}$, whether distinct or not. In Chapter 6 , based on the semantics proposed in [25] for Datalog with aggregation, we will formalize the query answering problem in EHM.

Example 6 (example 3 continued) The following Datalog with aggregation query computes total sales for different countries:

$$
Q(\text { ctr }, \operatorname{Sum}(\text { sales })) \rightarrow F(\text { cty, day, sales }), \mathcal{S}_{\text {City }}^{\text {Country }}(\text { cty, ctr })
$$

Here, $F$ is a relation that represents the fact table.

\subsection{Summarizability}

A common technique for speeding up OLAP query processing is to pre-compute some cube views and use them for the derivation (or answering) of other cube views. This approach to query answering is correct under the summarizability property, which ensures that higherlevel cube views (from the base categories and fact tables) can be correctly computed using cube-views at lower level as if they were fact tables [43]. As shown in Example 7, the reuse of pre-computed cube views has a great impact on query answering performance. Therefore, maximizing summarizability between categories is a crucial optimization task for MDDBs [46]. 
Example 7 Lets assume the Sales database of Figure 1.1 belongs to a worldwide corporation. The managers of this corporation need to compute its aggregate sales information for every continent between the years 2003 and 2012.

Computing this query directly (i.e. without taking advantage of summarizability) requires a join between three relations: $\mathcal{S}_{\text {City }}^{\text {Continent }}, \mathcal{S}_{\text {Day }}^{\text {Year }}$ and the fact table. Assuming the fact table contains daily values for 3000 cities over this period of time, these relations will contain more than 3000,3600 and $10^{7}$ tuples respectively. Obviously this will be a time consuming query.

On the other hand, if we were to compute the same query using a pre-computed cube view, for example $\Psi_{\text {sum }}[$ Country, Year], relations that were involved in the new join would be $\mathcal{S}_{\text {Country }}^{\text {Continent }}, \mathcal{S}_{\text {Month }}^{\text {Year }}$ and $\Psi_{\text {sum }}$ [Country, Year] with at most 200, 120 and 24000 tuples respectively. Hence, great improvement in query processing time would be obtained.

This performance improvement would be much more significant in real world examples involving more than two dimensions (e.g. by adding a product dimension) and more detailed base categories (e.g. a Location dimension with Branch as the base category rather than City).

As stated earlier, Reusing cube views is one the important features of multidimensional databases. The following query (Lets call it $C V^{c_{1}, \ldots, c_{n}}$ ) shows a template for writing cube views in MDX (for simplicity, some features of MDX that are irrelevant to our discussions have been dropped in this template):

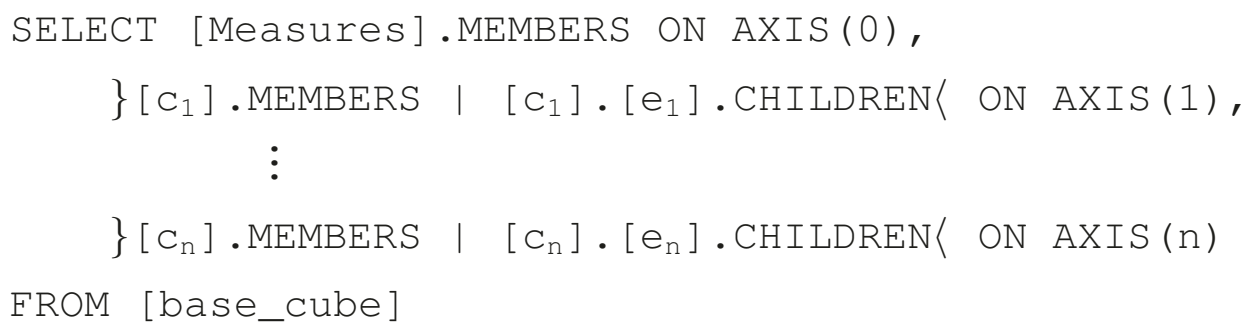

Here, $c_{1}, \ldots, c_{n}$ are categories (possibly from different dimensions), $\left[c_{k}\right]$. MEMBERS retrieves all elements that belong to category $c_{k}$ ([Measures].MEMBERS retrieves fact measures), $\left[c_{k}\right] \cdot\left[e_{k}\right]$. CHILDREN retrieves children of element $e_{k}$ from category $c_{k}$, and base_cube is a cube generated by selecting base categories of different dimensions (i.e. the fact table). 
The following query template can be used to derive cube view $C V^{c_{1}^{\prime}, \ldots, c_{n}^{\prime}}$ from another pre-computed cube view $C V^{c_{1}, \ldots, c_{n}}$ in MDX:

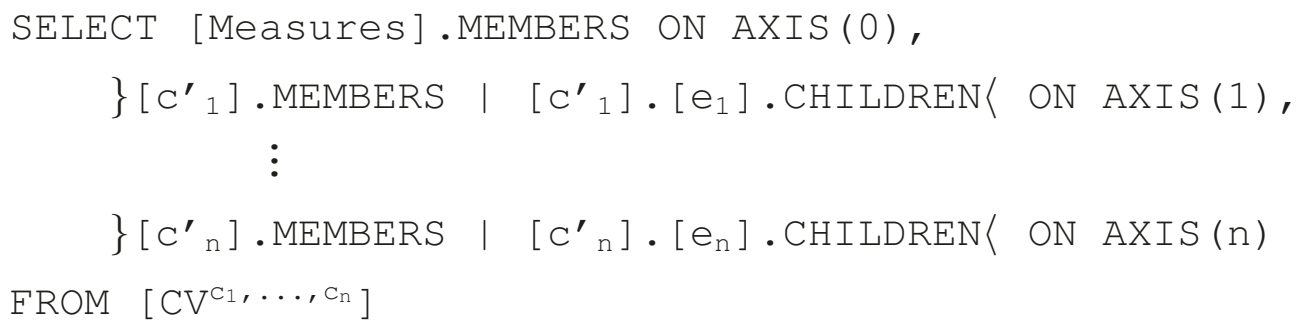

where $c_{1}, \ldots, c_{n}, c_{1}^{\prime}, \ldots, c_{n}^{\prime}$ are categories from different dimensions and every $c_{i}^{\prime}$ is an ancestor of $c_{i}$.

An HM instance is considered to be summarizable if a cube view on any category $c$ can be computed a from pre-computed cube view for each one of its descendant categories $c^{\prime}$ (as if the cube view for $c^{\prime}$ were a fact table) $[13,15,20,21,41,72]$. We will refer to this notion of summarizability (i.e. the one used in the literature) as strict-summarizability, as we indicated in Chapter 1. The reason for this is that in Section 5.1 we will relax this notion, providing a less restrictive definition of summarizability.

Definition 1 [72] An HM instance $\mathcal{H}$ is (a) Strict iff for all categories $c_{i}, c_{j}$, the roll-up relation $\mathcal{S}_{c_{i}}^{c_{j}}(\mathcal{H})$ is a (possibly partial) function. (b) Homogeneous iff for all categories $c_{i}, c_{j}$, the roll-up relation $\mathcal{S}_{c_{i}}^{c_{j}}(\mathcal{H})$ is total. (c) Consistent iff it satisfies the two previous conditions; and inconsistent otherwise.

Theorem 1 [41] An HM instance with a single base category is strictly-summarizable iff it is consistent.

\subsection{Data Repairs of MDDBs}

Data repairs have been introduced and studied in $[13,15,19,20,72]$, and in some sense, but not as repairs, in $[40,65]$. In this case, changes are made to the instance (as opposed to the schema) of an inconsistent dimension to restore consistency. In the following definition, $\operatorname{dist}\left(\mathcal{H}, \mathcal{H}^{\prime}\right)$ denotes the symmetric difference between the sets of links in instances of dimensions $\mathcal{H}$ and $\mathcal{H}^{\prime}$, i.e. $\operatorname{dist}\left(\mathcal{H}, \mathcal{H}^{\prime}\right)=\left(\begin{array}{ll}<_{\mathcal{D}} & <_{\mathcal{D}^{\prime}}\end{array}\right) \cap\left(\begin{array}{ll}<_{\mathcal{D}^{\prime}} & <_{\mathcal{D}}\end{array}\right)$. 

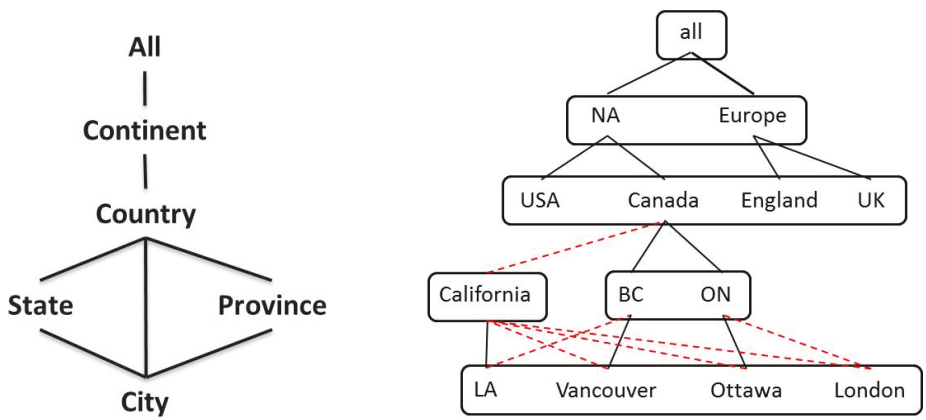

Figure 2.1: A minimal data repair for the Location dimension of Figure 1.1

Definition 2 [20] Given an $\mathrm{HM}$ instance $\mathcal{H}=\rangle \mathcal{Q}, \delta,<\mid$ over a schema S: (a) a data repair of $\mathcal{H}$ is an $\mathrm{HM}$ instance $\left.\mathcal{H}^{\prime}=\right\rangle \mathcal{Q}{ }^{\prime}, \delta^{\prime},<^{\prime} \mid$ over $\mathfrak{S}$ such that $\mathcal{Q}=\mathcal{Q}^{\prime}, \delta=\delta^{\prime}$ and $\mathcal{H}^{\prime}$ is consistent. (b) a minimal data repair of $\mathcal{H}$ is a data repair $\mathcal{H}^{\prime}$ such that $\backslash \operatorname{dist}\left(\mathcal{H}, \mathcal{H}^{\prime}\right) \backslash$ is minimal among all the repairs of $\mathcal{H}$.

Notice that data repairs (as defined above) have the same elements as the original dimension. They do not remove elements, otherwise a repair could contain less elements in the bottom category, and therefore, data from the fact tables would be lost in the aggregations. Also, they do not introduce new elements into a category. These new elements would have no clear meaning, and would not be useful when posing aggregate queries over the categories that contain them. For example, it is not clear what is the meaning of a new element in a category Month.

Example 8 Figure 2.1 shows a minimal data repair for the Location dimension of Figure 1.1. The schema remains the same as before. Three links have been removed, and six have been added to restore consistency. Notice that we could have obtained a different data repair by keeping the link between California and US and instead removing the links between BC, ON and Canada plus adding a link from BC, ON to US. However, that would result in more changes in the set of links, therefore it would not be minimal.

In [20], it has been shown there always exists at least one data repair (and minimal data repair) for any inconsistent HM instance, provided that the dimension has at least one element in each category. Also, there might exist more than one minimal data repair for an inconsistent HM instance. 
Theorem 2 [20] Let $\mathcal{H}$ be an HM dimension and $k$ be an integer. The problem of deciding whether there exists a data repair $\mathcal{H}^{\prime}$ of $\mathcal{H} \operatorname{such}$ that $\backslash \operatorname{dist}\left(\mathcal{H}, \mathcal{H}^{\prime}\right) \backslash \geq k$ is NP-complete.

It follows form the above theorem that the problem of computing a minimal data repair for an inconsistent HM instance is NP-hard. Thus, finding such a repair can be a computationally impractical task.

Another possible drawback of the data repair approach is, that for some real world domains, such as the one in Example 1, it may produce an unnatural solution, e.g. the data repair of Figure 2.1. Notice that connecting California to Canada is not an acceptable change in real applications.

\subsection{Related Work: State of the Art}

\subsubsection{Multidimensional Data Models}

Traditional data models, such as the ER model, are not well suited for OLAP applications. Therefore, new models that provide a multidimensional view of data have been created. They often categorize data as either measurable business facts, which are numeric in nature, or dimensions, which are mostly textual and characterize the facts. Various MD models have been proposed and investigated in the literature. These models can be divided into two general categories: simple cube models or hierarchical models.

The simple cube models $[27,30,31,48]$ view data as n-dimensional cubes. Each dimension has a number of attributes (or categories), which can be used for selection and grouping. For example, a Location dimension will have attributes such as City, State, Country, etc. These models remain as close to the standard relational model as possible, hence the theory and techniques developed for the relational model will be applicable. On the other hand, the hierarchy between the attributes is not captured explicitly by the schema of the simple cubes. So, from the schema, it cannot be learned for example that City rolls up to State and State to Country.

Hierarchical models $[3,18,28,43,47,51,59,66,71]$ explicitly capture the structural relationship between categories in the dimensions. The hierarchies are useful for navigating data cubes and also query optimization. They are usually captured by merging functions 
[3], lattices [18, 43, 66, 71], measure graphs [28], directed acyclic graphs [47], tree structures [51], or multidimensional extensions of the ER model [59].

Although extensive efforts have been made to propose MD models that fulfil OLAP requirements, many common issues have not been properly addressed:

Explicit and expressive hierarchies: As stated earlier simple cube models do not explicitly capture the hierarchy between categories. Hierarchical models provide explicit support, however in many cases this is just partial support because of the restrictions imposed by the chosen structures. Some models $[18,43,51,71]$ assume a single bottom-level attribute that is related to facts (usually through fact tables) which is a restricting assumption since many real-world scenarios require otherwise (e.g. in a Location dimension we may have different types of City, namely those with or without State, as multiple bottom-level categories). Some of the hierarchical models [3, 28, 51, 59] do not provide flexible classification structures to support unbalanced hierarchies (i.e. hierarchies with varying path-length from the generic top level categories to the bottom-level category (or categories)).

Non-strict dimensions: Most MD models [18, 28, 31, 47, 48, 51, 71] require hierarchies to be strict. This means that there should be no many-to-many relationship between the different levels in a dimension. This is despite the fact in real-world scenarios many-to-many relationships happen often. For example, in employee hierarchy, an employee may work under the supervision of more than one manager.

Heterogeneous dimensions: Most MD models have limited [43, 66] or no support $[3,18$, $28,31,48,51,59,71]$ for heterogeneous dimensions in which some elements have no parent in specific categories of the hierarchy.

Support for selecting pre-aggregated cube views: An important issue in improving query answering performance for MD databases is to choose a set of cube views for materialization when it is too expensive to materialize all cube views [33]. Later, when another aggregate query needs to be computed, we need to be able to decide whether this new query can be computed using the set of materialized pre-computed cube views (and if so, which ones). 
We will refer to this problem as the pre-aggregated cube view selection. Most existing MD models make the restrictive assumption of having strict and homogeneous dimensions with a single bottom-level attribute and as a result avoid having to deal with this problem (because with this highly restrictive assumption every cube view can be derived from any other pre-computed cube view). Some efforts have been made to tackle this problem $[33,63]$ but because of the lack of support in the modeling layer, the resulting solutions are not efficient.

\subsubsection{Summarizability}

A common technique for speeding up OLAP query processing is pre-computing some cube views and use them for the derivation (or answering) of other cube views. This approach to query answering is correct under the summarizability property, which ensures that higherlevel cube views (from the base categories and fact tables) can be correctly computed using cube-views at lower level as if they were fact tables [43]. The reuse of pre-computed cube views has a great impact on query answering performance. Therefore, maximizing summarizability between categories is a crucial optimization task for MDDBs [46].

Summarizability has been defined in slightly different ways in the literature [35, 43, 53]. More importantly, there have been efforts to characterize the concept by providing semantic conditions that are required for or/and guaranty summarizability [50, 52, 53]. Also, several techniques for deciding, enforcing or restoring these conditions have been investigated $[35,38]$. We will briefly give an overview of some of these works.

\section{Definition and Characterization of Summarizability}

In [53], the authors define summarizability as the guaranteed correctness of aggregation results. They discuss the importance of this property for MD queries and argue that any MD schema should be set up in a way that summarizability is obtained to the highest possible degree. Furthermore, if this property is violated along certain aggregation paths, the schema should clearly express the restriction to avoid incorrect aggregation results. Authors of [53] provide necessary conditions for summarizability that can be regarded as a first step towards quality of data warehouse schemata. In particular, they argue that elements of each level in a dimension instance must be complete (i.e. cover all elements of 
the bottom level) and disjoint, i.e. partitioning.

The authors of [43] define summarizability as the ability of an aggregate query to correctly compute a cube view from another cube view where both cube views are defined in a single dimension. They formally characterize (local) summarizability of category $c$ from a set of its descendants $S$ as follows: every base member (i.e., a member in a bottom category) that rolls up to $c$, rolls up to $c$ passing through one and only one of the categories in $S$. This is actually expressed in formal terms by a constraint language defined in [43]. It is also shown that the lattice-based schema of the HM model itself is not expressive enough to support summarizability reasoning (i.e. determining the summarizability of categories from descendants) in the presence of heterogeneity. In order to overcome this limitation, a class of integrity constraints have been defined as part of the schema (known as dimension constraints).

In [35], summarizability is (informally) defined as the property of whether performing an aggregate operation will result in an accurate result. Potential summarization problems are categorized into schema level problems (including multiple path, non-strict and heterogeneous hierarchies), data level problems (including imprecise, biased and inconsistent measurements), and computational problems (including unit differences and sample size issues).

In [62], the authors identify three parts of an aggregate query as: the aggregation operation, the measure that is to be aggregated, and either an aggregation level or a member for each dimension. They argue that a query can only produce meaningful results if: (1) The aggregation operation is appropriate for the measure, and (2) The measure is appropriate for the aggregation levels in the cube's dimensions. The selected data is called summarizable with respect to the aggregation operation if both conditions hold.

\section{Deciding and Enforcing Summarizability}

In [38], it has been shown that testing summarizability in the HM model (i.e., deciding whether a category is summarizable from a set of categories in a dimension schema) is coNP-complete. Also, given a category, determining whether it is summarizable from any subset of a given set of descendants is NP-hard.

The authors of [52] define generalized multidimensional normal forms (GMNF) that 
allow to perform schema design for data warehouses, in the spirit of classical database design. A schema in GMNF ensures summarizability in a context-sensitive manner and supports an efficient physical database design by avoiding inapplicable null values. More specifically, GMNF addresses the following issues: First, the occurrence of inapplicable null values indicates a conceptual flaw in the schema design, and such null values should be avoided. Second, the presence of inapplicable null values may lead to the formulation of contradictory queries.

The authors of [50] argue that the normal forms proposed in [52] are too restrictive because they rule out certain desirable fact schemata, and at the same time allow undesirable schemata. They propose three MD normal forms in increasing order of restrictiveness that satisfy different objectives. 1MNF captures three orthogonal design objectives, namely faithfulness, completeness, and freedom of redundancies. 2MNF strengthens $1 \mathrm{MNF}$ by taking care of optional dimension levels in order to guarantee summarizability in a contextsensitive manner. Finally, 3MNF places further restrictions that are sufficient to construct a class hierarchy concerning dimension levels, which is implicitly contained in a fact schema, in terms of relation schemata that avoid null values.

\section{Restoring Summarizability}

The authors of [35] try to address situations where systems are not optimally structured or conceptual models are not consulted and argue that it is important to make the impact of structural violations apparent at query time. They propose to run scripts at query time to identify summarization issues. For example, in the case of a non-strict hierarchy, they propose to run scripts at query time to identify the number times measures are counted in the summary and identify the total value of any duplicated values.

A more common approach towards restoring summarizability is to make changes to the dimension schema and/or the dimension instance to restore strictness and homogeneity. In accordance with the area of consistent query answering (CQA) in relational databases $[7,11,12]$, a resulting dimension instance could be called a repair of the original one. A minimal repair (we usually and simply call it a "repair") is one that minimally differs (in some prescribed sense of minimality) from the original dimension and satisfies strictness and homogeneity $[9,13]$. 


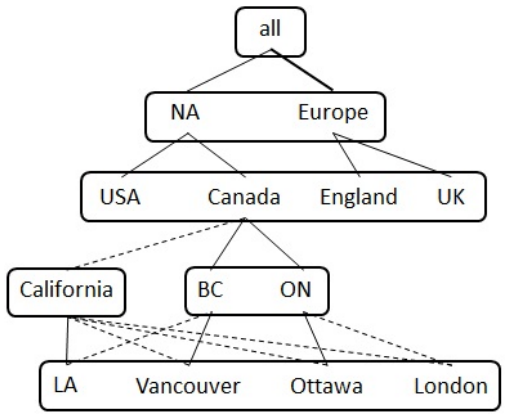

(a) Data repair by changing links

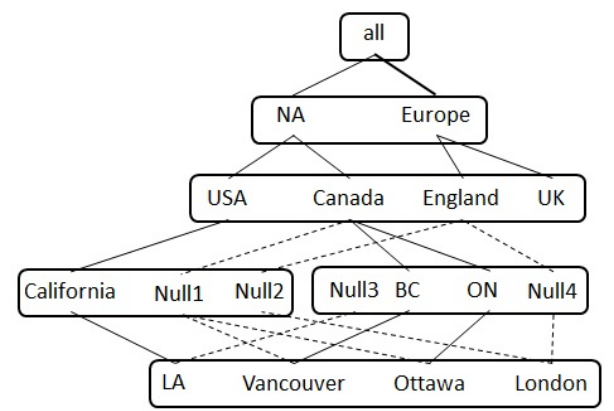

(b) Data repair by adding nulls

Figure 2.2: Data repairs of the Location dimension

In [19], preliminary work on the effect of non-strictness on aggregate queries from homogeneous dimensions was carried out. The focus is on finding ways to retrieve consistent answers even when the MDDB is inconsistent. To restore consistency, links and elements in the dimension instance are deleted in a minimal way.

The notion of (data) repair of an MDDB was introduced in [13]. Data repairs are generated by adding and removing links between data elements, for restoring strictness on already homogeneous dimensions. Inspired by [8], consistent answers to aggregate queries on non-strict dimensions are characterized in terms of a smallest range that contain the usual (numerical) answers obtained from the minimal data repairs.

In [15], repairs of heterogeneous or non-strict dimensions (or both) are obtained by insertions and deletions of links between elements. Adding a link in the instance to remove heterogeneity may cause non-strictness in parts of the instance that did not have any problems before. Therefore, this has to be done in an iterative manner until everything is fixed (usually after many changes). Logic programs with stable model semantics were proposed to represent and compute minimal dimension repairs. In [20], the authors present a complexity analysis of the problem of computing a minimal repair, and show that in general the problem is NP-hard. However, they show a specific case in which this can be done in polynomial time. They also prove that repairs always exist.

In [21], a similar data repair approach is studied with the assumption that non-strictness is caused by updating links in the dimension instance and the repair should not be allowed to undo the link update. Hence, only minimal data repairs that restore strictness without changing specific links are considered (these are called $r$-repairs). 
In [65], methodologies and algorithms are given for transforming inconsistent dimensions into strict and homogeneous ones. The dimension instance is changed (as opposed to the schema). Problems caused by inconsistent dimensions are fixed by introducing NULL members as new data elements. In [40], the authors study the implications of relaxing the homogeneity condition on MDDBs. They also argue that the addition of NULL values (elements) and changes to the schema provide a solution to structural heterogeneity.

In [72], as part of our research (but not of this thesis), we address non-summarizability through a new relational representation of MDDBs (i.e. the Path Relational Schema). We start by representing our original MDDB as a relational database, through an MD2R mapping that translates the multidimensional data model (MDM) into an adequate relational model. The latter includes a schema that allows for the representation of the MDDB conditions of strictness and homogeneity as relational integrity constraints (ICs). The translation is such that the original MDDB is inconsistent, iff the resulting database is inconsistent wrt the created ICs. Next, the resulting inconsistent relational instance is repaired as a relational database, using existing techniques [12]. As a result, we obtain a set of minimal relational repairs.

Data repairs have been the main focus of previous work on repairing MDDBs. However, these repairs aren't usually the ideal solution. One drawback of data repairs is that they manipulate the dimension instance, i.e. the data. In case the MDDB is implemented on a relational $\mathrm{DB}$, this results in changes in records, which users may find undesirable.

Another problem with data repairs is that resolving heterogeneity may require introducing redundant NULL data values in the dimension instance. Those values have to be proliferated along the path of ancestor categories. The alternative approach for removing heterogeneity that is based on insertions of new links has the problem of possibly introducing new sources of non-strictness, which have to be resolved. This may introduce multiple changes in the dimension instance.

In addition, there exist real world domains, such as the Location dimension of Figure 1.1, where this approach may produce an unnatural solution, e.g. one that connects London to California in State (as shown in Figure 2.2a), or a null in State (as with Figure $2.2 b$ ). Of course, additional restrictions could be imposed on the repair process, like not 
allowing such links, but in some cases there might be no repair then.

\subsubsection{Cube View Selection}

In a multi-dimensional database, query response can be significantly improved by an appropriate selection of cube views to be pre-computed for reuse. Cube view selection involves two major decisions:

1. A decision has to be made about which cube views to pre-compute and materialize. Obviously, materialization of all cube views is unfeasible, both because of their size, and of the time required to update them when the fact table is updated.

2. Having a query and a set of materialized cube views, we need to choose a cube view (or a set of cube views) from which the query result can be correctly derived.

In [10], the authors address the problem of cube view materialization assuming a set of user-specified relevant queries is available. The indication of relevant queries is exploited to drive the set of candidate views that, if materialized, may yield a reduction of the total cost. A formal characterization of cost and total materialization cost is also provided.

In [63], the problem of cube view materialization in data warehouses is treated as a variant of the basic problem of space constrained optimization. The authors explore the use of greedy and randomized techniques for this problem.

In [5], the clustering technique of data mining is exploited to address the problem of materialized cube view selection. In addition, the authors propose a view merging algorithm that builds a set of candidate views, as well as a greedy process for selecting a set of views to materialize.

The works mentioned above, among others [57, 68], focus on one of the problems in cube view selection, i.e. deciding which cube views to materialize (as opposed to finding query results using available views). However, in [54] the authors consider the problem of finding a rewriting of a conjunctive query using available materialized views. They describe algorithms for solving different forms of the problem including finding minimal rewritings, and finding complete rewritings (i.e. rewritings that only use the views). Particularly, it is shown that all the possible rewritings can be obtained by considering containment mappings from views to the query. In [54], queries and views do not contain aggregation. 
In the case of MDDBs, we can restrict our focus to aggregate queries (as opposed to conjunctive queries that are investigated in [54]). In Chapter 6, we will address the second problem mentioned above for MD aggregate queries. Our approach to query answering using views can be considered a complement for previous work in cube view materialization. 


\section{Chapter 3}

\section{Structural Repairs of Multidimensional Databases}

\subsection{Structural Repairs}

To restore consistency (i.e. homogeneity and strictness) for an HM dimension, changes have to be made to the dimension schema and/or the dimension instance. Previous work on repairing MDDBs has focused mainly on changing the dimension instance by modifying data elements or links between them (see Section 2.4). However, inconsistency may be caused by a problematic dimension schema. Therefore, changing the dimension instance may be only a temporary solution. Future update operations will probably produce new inconsistencies. So, exploring and considering structural changes, i.e. changes in the schema, is a natural way to go. In [9], we introduce and investigate the notion of structural repair, as an alternative to data repair. They modify the schema of an inconsistent dimension, to restore strictness and homogeneity. To the best of our knowledge, this is the first formal treatment of schema-based repairs.

Structural repairs restrict changes to dimension instances by allowing changes that affect only the category lattice and the distribution of the data elements in different categories. In particular, there are no "data changes" (as in data repairs), in the sense that the data elements and also the edges between them remain. In the case of a ROLAP data warehouse (with relational schemas, like star or snow flake) fewer data changes on a dimension instance directly results in fewer changes on records in the underlying database.

Definition 3 A structural repair for an inconsistent $\mathrm{HM}$ instance $\mathcal{H}=\rangle \mathcal{Q}, \delta,<\mid$ over schema $\mathfrak{S}=\rangle U, \mathcal{D}, \Rightarrow \mid$ is a pair $\rangle \mathcal{H}^{\prime}, g \mid$, with $\left.\mathcal{H}^{\prime}=\right\rangle \mathcal{Q}, \delta^{\prime},<\mid$ another HM instance over schema $\left.\mathfrak{S}^{\prime}=\right\rangle U, \mathcal{D}, \Rightarrow^{\prime} \mid$, with the following properties:

(a) $\mathcal{H}^{\prime}$ is strict and homogeneous, i.e. consistent.

(b) Elements cannot be moved between categories that exist both in $\mathcal{H}$ and $\mathcal{H}^{\prime}$ : For all $e, c_{1}, c_{2}$, if $\delta(e)=c_{1}, \delta^{\prime}(e)=c_{2}, c_{1} \vDash c_{2}$, then $\} c_{1}, c_{2}\langle\nsubseteq(\mathcal{D}\{\mathcal{D})$. 


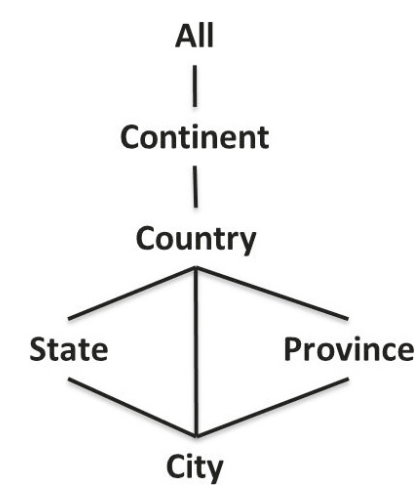

(a) Location schema

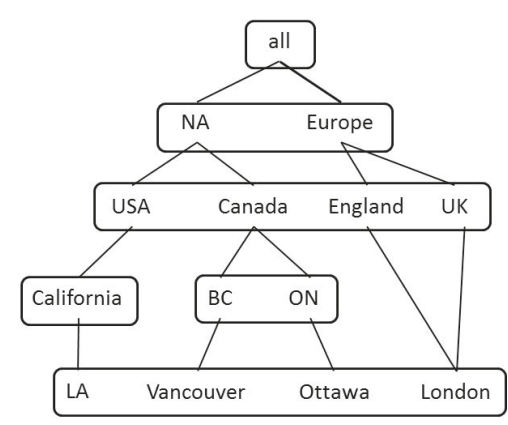

(b) Location instance

(c) Any new category in $\mathcal{H}^{\prime}$ must have at least one data element.

(d) $g: \mathcal{D} \nearrow 2^{\mathcal{C}^{\prime}}$, the schema mapping, is a total function that maps each category of $\mathcal{H}$ to a set of categories in $\mathcal{D}$ (which is finite in $\mathcal{H}^{\prime}$ ).

(e) If $c^{\prime} \triangle g(c)$, then $c$ and $c^{\prime}$ share at least one data element.

The role of $g$ is to establish a relationship between the schemas of $\mathcal{H}$ and $\mathcal{H}^{\prime}$. For each category $c$, the set of its "elements", i.e. $\} e \backslash \delta(e)=c\langle$, may be $\leq$-incomparable with \}$e \backslash \delta(e)=c^{\prime}$ and $c^{\prime} \triangle g(c)\langle)$.

Condition (b) in Definition 3 ensures that in a structural repair, data elements move from one category to another only as a result of changes made to the dimension schema (splitting or merging categories of $\mathcal{H}$ ). ${ }^{1}$

Figures 3.1 and 3.2 show two of the possible structural repairs for the inconsistent Location dimension of Figure 3.1. They show that strictness forces us to put elements England and UK in different categories. On the other hand, homogeneity forces us to either merge categories State and Province or isolate element LA in a new category.

Proposition 1 Every inconsistent dimension has a structural repair.

Proof: This proposition can be proved by using a simple structural repair that creates a new one-element category for each element in the dimension instance, and also links between the newly created categories whenever their single elements are connected in the original instance. In the schema of this structural repair there exists one category for every data element and one link for each data link in the dimension instance that connects the

\footnotetext{
${ }^{1}$ Isomorphic structural repairs, i.e. that differ only in the names of active categories, will be treated as being the same repair.
} 

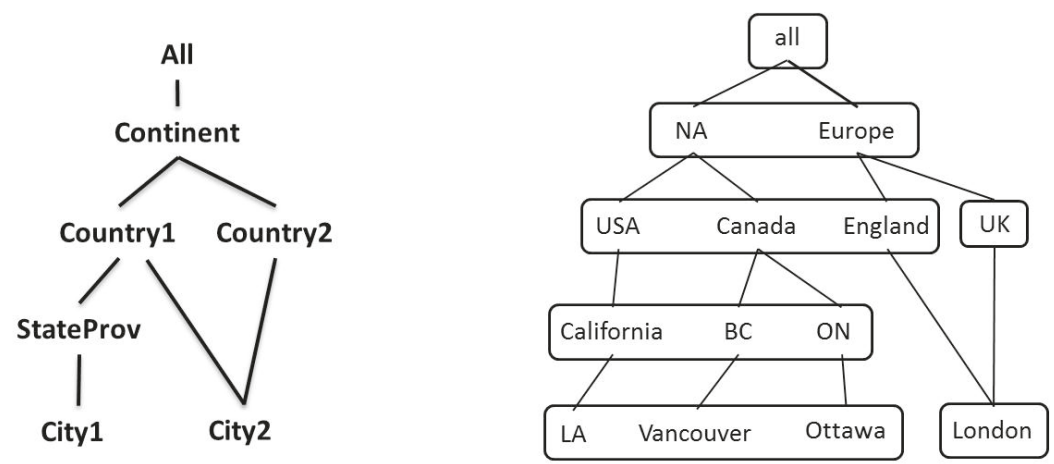

Figure 3.1: An SR for the Location dimension

corresponding categories. It can easily be shown that this new dimension is strict and homogeneous. First of all, every category in the new dimension contains exactly one element, and therefore this dimension is obviously strict. On the other hand, we know that links in the dimension schema have been added according to the links that exist between corresponding elements in the dimension instance. Therefore, whenever a category $c_{1}$ rolls up to category $c_{2}$, the single data element that belongs to $c_{1}$ also rolls up to the single element that belongs to $c_{2}$ which proves homogeneity.

Example 9 The following mapping is a schema mapping between the Location dimension of Figure 1.1 and the structural repair of Figure 3.1:
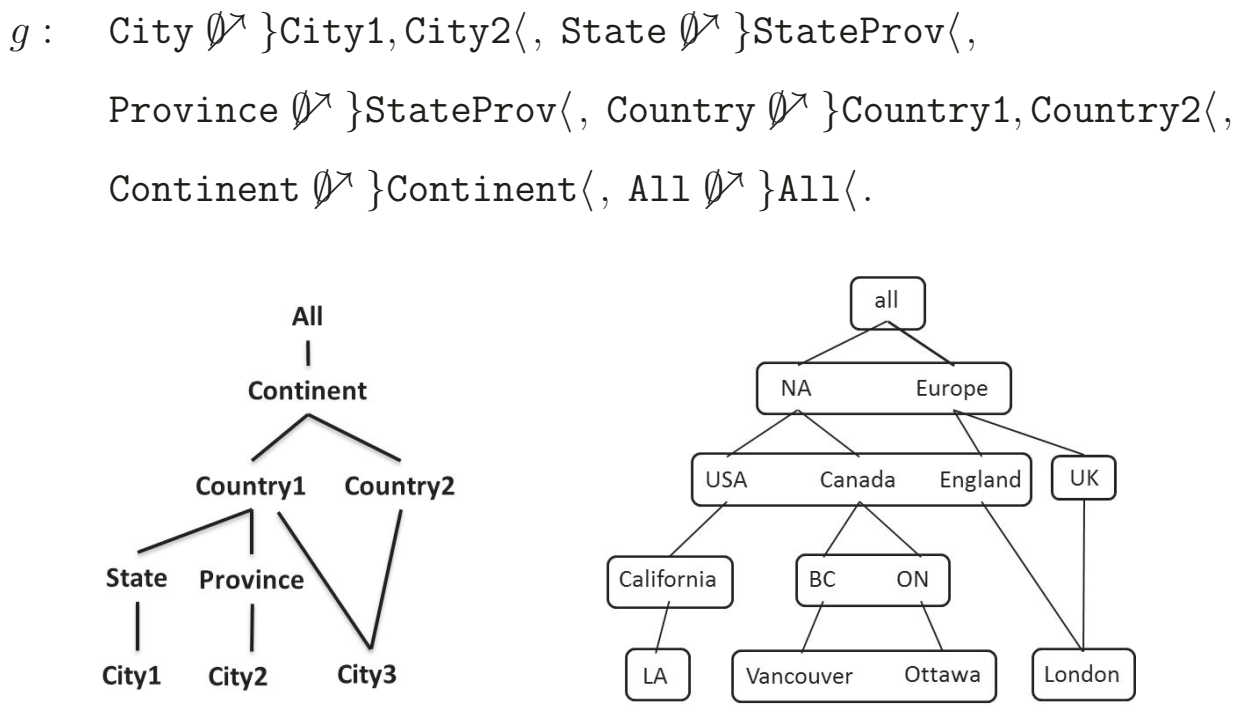

Figure 3.2: Another SR for the Location dimension 
Notice in this example the satisfaction of condition (b) from Definition 3: elements have moved from old categories (e.g. State) to new categories (e.g. StateProv). However, no element has moved from a category that exists in both the dimension and its SR (e.g. no element has moved from Continent to any other category).

Next, we define minimal structural repairs (MSRs), as preferred repairs among the structural repairs. This requires comparing structural repairs. The data movement set, defined next, has useful properties for this comparison (cf. Section 3.2).

Definition 4 Let $\rangle \mathcal{H}^{\prime}, g \mid$ be an SR for dimension instance $\mathcal{H}$, and $c$ be a category of $\mathcal{H}$.

(a) The data movement set of category $c$ is defined by:

$$
\left.\operatorname{DMSet}_{g}(c)=\right\} e \backslash \delta(e)=c\left\langle/ \bigcup_{c^{\prime} \in g(c)}\right\} e \backslash \delta(e)=c^{\prime}\langle
$$

where / denotes the symmetric difference of two sets.

(b) The overall data movement set between $\mathcal{H}$ and $\mathcal{H}^{\prime}$ is defined by

$$
\operatorname{DMSet}_{g}\left(\mathcal{H}, \mathcal{H}^{\prime}\right):=\bigcup_{c \in \mathcal{C}} \operatorname{DMSet}_{g}(c)
$$

Categories of a dimension and its SR will be mapped by a schema mapping. The data movement set of a category contains the difference between elements that belong to that category in the original dimension and the ones that belong to its set of mapped categories in the SR.

Example 10 (example 9 cont.) For the schema mapping in the example:

$$
\begin{aligned}
& \text { DMSet }_{g}(\text { City })=\text { LLA, Ottawa, Toronto, London }\langle/ \text { \}LA, Ottawa, Toronto, London }\langle \\
& =\mathcal{C}, \\
& \operatorname{DMSet}_{g}(\text { State })=\text { California }\langle/ \text { California, } \mathrm{BC}, \mathrm{ON}\langle=\} \mathrm{BC}, \mathrm{ON}\langle\text {, } \\
& \text { DMSet } \left._{g}(\text { Province })=\right\} \text { BC, ON }\langle/ \text { California, } \mathrm{BC}, \mathrm{ON}\langle=\} \text { California }\langle\text {, } \\
& \text { DMSet }_{g}(\text { Country })=\text { \}US, Canada, England, UK }\langle/\} \text { US, Canada, England, UK }\langle=\mathcal{C} \text {, } \\
& \text { DMSet } \left.\left._{g} \text { (Continent }\right)=\right\} \text { NA, Europe }\langle/\} N \text { A, Europe }\langle=\mathcal{C}, \\
& \left.\operatorname{DMSet}_{g}(\mathrm{All})=\right\} \operatorname{All}\langle/\} \operatorname{All}\langle=\mathcal{C} .
\end{aligned}
$$


Intuitively, an MSR is a new dimension that is obtained by applying a minimal set of changes to the schema of an inconsistent dimension. Inspired by the notion of prioritized minimization [56], we propose to minimize both data movement and the changes in the set of categories, but assigning higher priority to minimizing the former.

Definition 5 For a dimension $\mathcal{H}$ and two SRs $\rangle \mathcal{H}_{1}^{\prime}, g_{1} \mid$ and $\rangle \mathcal{H}_{2}^{\prime}, g_{2} \mid$ :

$$
\begin{aligned}
\left\langle\mathcal{H}_{1}^{\prime}, g_{1} \mid \geq_{\mathcal{D}}\right\rangle \mathcal{H}_{2}^{\prime}, g_{2} \mid \text { iff } \operatorname{DMSet}_{g_{1}}\left(\mathcal{H}, \mathcal{H}_{1}^{\prime}\right) \leq \operatorname{DMSet}_{g_{2}}\left(\mathcal{H}, \mathcal{H}_{2}^{\prime}\right) \text { and } \\
\qquad \operatorname{DMSet}_{g_{1}}\left(\mathcal{H}, \mathcal{H}_{1}^{\prime}\right)=\operatorname{DMSet}_{g_{2}}\left(\mathcal{H}, \mathcal{H}_{2}^{\prime}\right) \Leftrightarrow\left(\mathcal{D}^{\mathcal{P}} / \mathcal{D}^{\mathcal{D}_{1}^{\prime}}\right) \leq\left(\mathcal{D}^{\mathcal{P}} / \mathcal{D}^{\mathcal{D}_{2}^{\prime}}\right) .
\end{aligned}
$$

Here, $\mathcal{D}^{\mathcal{D}}, \mathcal{D}^{\mathcal{P}_{1}^{\prime}}$ and $\mathcal{D}^{\mathcal{P}_{2}^{\prime}}$ denote the finite sets of categories for dimensions $\mathcal{H}, \mathcal{H}_{1}^{\prime}$ and $\mathcal{H}_{2}^{\prime}$, respectively.

Definition $6>\mathcal{H}_{1}^{\prime}, g_{1} \mid$ is a minimal structural repair (MSR) of dimension $\mathcal{H}$ iff it is an SR of $\mathcal{H}$ and there is no other SR $\rangle \mathcal{H}_{2}^{\prime}, g_{2} \mid$ for $\mathcal{H}$, such that $\rangle \mathcal{H}_{2}^{\prime}, g_{2}\left|<_{\mathcal{D}}\right\rangle \mathcal{H}_{1}^{\prime}, g_{1} \mid$. (Here, as expected, $u<_{\mathcal{D}} v$ means $u \geq_{\mathcal{D}} v$, but not $v \geq_{\mathcal{D}} u$.)

Example 11 It can be shown that the structural repair of Figure 3.2 is an MSR for the inconsistent Location dimension of Figure 1.1, with the following schema mapping:

$$
\begin{aligned}
& g_{1}: \operatorname{City} \emptyset \nearrow \text { City1, } \operatorname{City} 2, \operatorname{City} 3\langle\text {, State } \emptyset \nearrow\} \text { State }\langle\text {, } \\
& \text { Province } \emptyset^{\top} \text { \}Province }\langle\text {, Country } \emptyset \text { \}Country1, Country2<, } \\
& \text { Continent } \left.\phi^{\top}\right\} \text { Continent }\left\langle, \operatorname{All} \phi^{\top}\right\} \operatorname{All}\langle\text {. }
\end{aligned}
$$

On the other hand, the structural repair of Figure 3.1 is not an MSR for any possible schema mapping. This is because for categories State and Province in the original dimension, there is no category (or set of categories) in this repair that contains exactly the data elements that belong to those two categories. Therefore, for any mapping $g_{2}$, $D_{M S e t}($ State $) \vDash \mathcal{C}$ and $D M S e t_{g_{2}}($ Province $) \vDash \mathcal{C}$. As a result, DMSet $_{g_{2}}$ (State $) \varsubsetneqq$ DMSet $_{g_{1}}$ (State). According to the definition of MSR, $g_{2}$ cannot be minimal.

Proposition 2 An inconsistent dimension always has a minimal structural repair.

Proof: This can be readily obtained from Proposition 1. In the worst case, a simple structural repair exists that essentially creates a new one-element category for each element in 

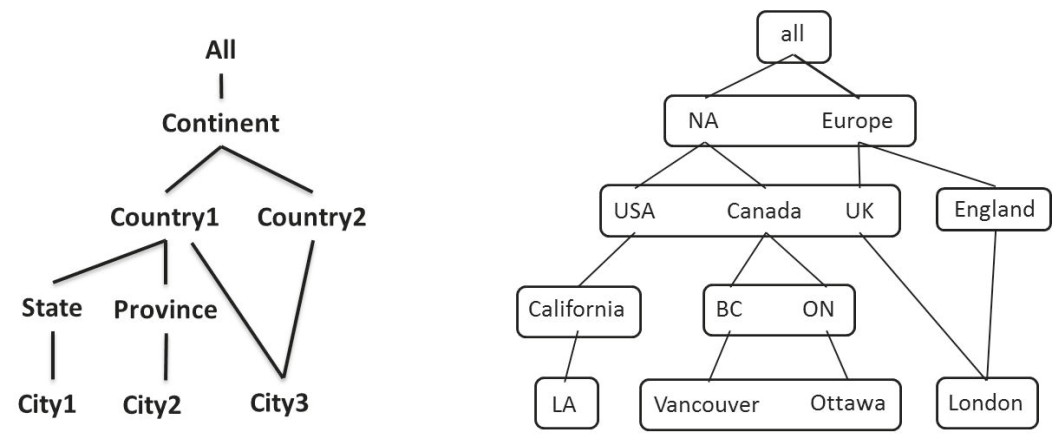

Figure 3.3: Another minimal SR for the Location dimension

the dimension instance, and also links between the newly created categories whenever their single elements are connected in the original instance. In the schema of this structural repair there exists one category for every data element and one link for each data link in the dimension instance that connects the corresponding categories.

The following example shows that an inconsistent dimension may have multiple MSRs.

Example 12 The structural repair of Figure 3.3, that is obtained by swapping the data elements England and UK in the structural repair of Figure 3.2, shows another minimal structural repair for the inconsistent dimension of Figure 1.1. These two minimal repairs are not isomorphic.

If the notion of MSR is applied to a consistent dimension $\mathcal{H}$, then $\rangle \mathcal{H}, i d \mid$ is one of its MSRs, with $\left.i d: c \emptyset^{\top}\right\} c\langle$, but not necessarily the only one. This is because we can have categories with no members in a consistent dimension, and changing the links that are attached to them results in different minimal repairs. To ensure that a consistent dimension is its only MSR, we have to add an extra condition in the definition of minimal repair, that would ensure that if two SRs have an empty data movement set, the one that has fewer category and link changes will be preferred. Otherwise, even a consistent dimension would possibly have multiple MSRs (including itself).

\subsection{Properties of Structural Repairs}

An OLAP system is usually modeled by a three level architecture consisting of a data warehouse server, a query client and a data cube engine [22]. The performance of an OLAP 
system is based on how it performs at each level. Any repair is expected to remove inconsistencies of a dimension, while maintaining good properties at each of the three levels. From this point of view, an ideal repair should satisfy the following requirements:

1. Limited changes to the underlying database: Changes to the underlying database forced by the transition from the original dimension to the repair should be as few as possible. By definition, structural repairs restrict changes to data so that elements and links in the dimension instance do not change at all. Therefore, the first requirement is strictly satisfied by the use of structural repairs.

2. Rewritability of cube views: Queries imposed on the original (inconsistent) HM dimension refer to categories that may not exist in the SR anymore. These queries should be rewritten in terms of new categories and the new queries should return equivalent outputs in terms of selected data elements and their respective aggregate values.

Next, we will formally define the notion of rewritability (Definition 7) and characterize the necessary conditions that make a query rewritable in an SR.

Definition 7 A cube view $\Psi$ on categories of dimensions $\mathcal{H}_{1}, \ldots, \mathcal{H}_{n}$ is rewritable on a set of SRs $>\mathcal{H}_{1}^{\prime}, g_{1}|, \ldots,\rangle \mathcal{H}_{n}^{\prime}, g_{n} \mid$ if there exists a cube view $\Psi^{\prime}$ on categories of the SRs, such that $\Psi$ and $\Psi^{\prime}$ are equivalent (i.e. produce the same answer for instances of given schemas). $\Psi^{\prime}$ is called a rewriting of $\Psi$ over the set of SRs.

The rewritability of a cube view depends on the expressive power of the query language being used. We will assume next that cube views are defined using a relationally complete language $[23,55]$.

Theorem 3 shows that to have rewritability for a cube view over a set of dimension schemas, the data movement set for all the categories involved in the cube view should be an empty set. Intuitively, an empty data movement set ensures that any category (from the original schema) and the set of categories (from the SR) replacing that specific category contain exactly the same elements and therefore a query and its rewriting will produce the same result.

Theorem 3 A cube view $\Psi_{f}\left[c_{1}, \ldots, c_{n}\right]$ on categories of dimensions $\mathcal{H}_{1}, \ldots, \mathcal{H}_{n}$ is rewritable on a set of SRs $\rangle \mathcal{H}_{1}^{\prime}, g_{1}|, \ldots,\rangle \mathcal{H}_{n}^{\prime}, g_{n} \mid$, if: 


$$
\operatorname{DMSet}_{g_{1}}\left(c_{1}\right)=\ldots=\text { DMSet }_{g_{n}}\left(c_{n}\right)=\mathcal{C}
$$

In particular, cube views are always rewritable over MSRs.

Proof: It suffices to show that every category in the initial set of dimensions can be regenerated by an operator on (elements of) categories of the structural repairs. if this can be done, we can rewrite cube view $\Psi_{f}\left[c_{1}, \ldots, c_{n}\right]$ by simply replacing every occurrence of any $c_{i}$ with the statement that regenerates its elements. Consider $c_{i}^{\prime}$ as a new category, with scope of a query, that contains $\} e \zeta \delta(e)=c_{i}^{\prime}\langle$ as its member elements and serves as a new category that replaces $c_{i}$. Since $\operatorname{DMSet}_{g}\left(c_{i}\right)=0$, it readily follows from Definition 4 that $c_{i}^{\prime}$ will have the exact same members as $c_{i}$. Therefore, cube view $\Psi_{f}\left[c_{1}, \ldots, c_{n}\right]$ can be rewritten in any query language that allows the union operation on data elements (and more specifically, in any relationally complete language).

Notice that in the theorem the inverse implication may not hold: Even for repairs with non-empty data movement set, some queries may be rewritable. This case happens when the query imposed on a category such as $c$ from the original schema involves conditions that filters out any element from $\operatorname{DMSet}_{g}(c)$ and therefore the rewriting will automatically discard those problematic elements (that would have made the queries non-equivalent).

Example 13 Consider the Location dimension of Figure 1.1 and a cube view (a) below for this dimension. Assume that Fact $\mathrm{s}$ is the fact table, and Sales is a fact measure. This cube view is not rewritable on the structural repair of Figure 3.1, and DMSet (Province) $^{\text {(P) }}$ $\vDash \mathcal{C}$. On the other hand, for the structural repair of Figure 3.2 (that has empty data movement set for all categories), the cube view in ( $b$ ) below is a rewriting. It is obtained by replacing $\mathcal{S}_{\text {City }}^{\text {Province }}$ by $\mathcal{S}_{\text {City2 }}^{\text {Province }}$.

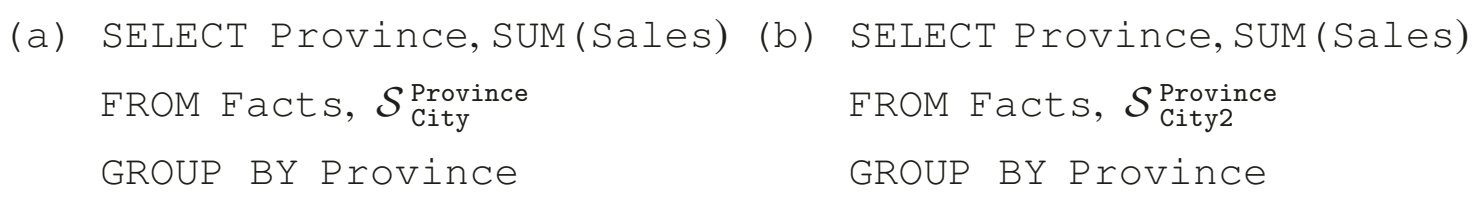

3. Preservation of summarizability: If the original dimension allows summarizations over existing cube views, to compute new cube views, similar summarizations should also be possible in the repaired dimension. 
Example 14 Consider the Location dimension of Figure 1.1 and a pre-computed cube view $\Psi_{\text {sum }}[$ Country] in (a) below for category Country. Also, consider cube view (b) below that uses $\Psi_{\text {sum }}[$ Country] to derive aggregate values for category All:

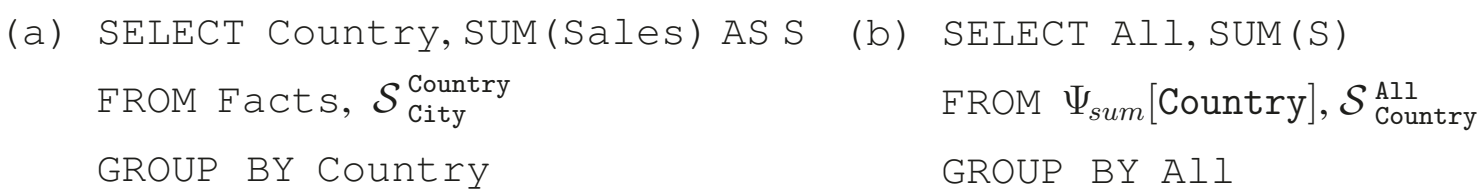

This derivation will produce correct results, only if category $\mathrm{All}$ is summarizable from category Country. In the original inconsistent dimension of Figure 1.1, this summarizability property does not hold. This is because in the instance of this dimension, element London will be considered twice as a descendant of all (via England and UK).

Cube view $\Psi_{1}$ depends on cube view $\Psi_{2}$ iff the former can be answered using the result of the latter [33]. For this to hold, each $c_{i}$ in $\Psi_{1}$ must be summarizable from a subset of categories in $\Psi_{2}$ (See Section 2.3 for A formal definition of category summarizability).

Definition 8 An SR $\rangle \mathcal{H}^{\prime}, g \mid$ for dimension $\mathcal{H}$ preserves summarizability of category $c$ over categories $\} c_{1}, \ldots, c_{n}\left\langle(\right.$ all in $\mathcal{H})$ iff every $c^{\prime} \triangle g(c)$ is summarizable from $\bigcup_{k=1}^{n} g\left(c_{k}\right)$ in $\mathcal{H}^{\prime}$.

Intuitively, when an SR preserves summarizability of a category $c$ over a set of its descendants, it means that cube views over the categories that replace $c$ are still derivable from the ones that replace its descendants in the SR.

Theorem 4 An SR $\rangle \mathcal{H}^{\prime}, g \mid$ for dimension $\mathcal{H}$ preserves summarizability of category $c$ over categories $\} c_{1}, \ldots, c_{n}\left\langle\right.$ if $\operatorname{DMSet}_{g}(c)=\operatorname{DMSet}_{g}\left(c_{1}\right)=\times \propto \times=\operatorname{DMSet}_{g}\left(c_{n}\right)=\mathcal{C}$. In particular, minimal structural repairs preserve all the summarizability properties.

Proof: The necessary and sufficient condition for category $c$ to be summarizable from categories $\} c_{1}, \ldots, c_{n}\langle$ is that all elements of bottom categories that roll up to some element in $c$, also roll up to exactly one element that belongs to $\} c_{1}, \ldots, c_{n}\langle$ (disjointness and completeness) [41]. We want to prove that every $c^{\prime} \triangle g(c)$ is summarizable from $\bigcup_{k=1}^{n} g\left(c_{k}\right)$ in $\mathcal{H}^{\prime}$. Let's assume $e_{\text {base }}$ is an element from a bottom category that rolls up to some element in $c^{\prime} \triangle g(c)$ (let's call it $\left.e_{a n c}\right)$. Clearly, in the original dimension $e_{\text {anc }}$ belongs to $c$ 
since $\operatorname{DMSet}_{g}(c)=\mathcal{C}$. We already know that $c$ is summarizable from $\} c_{1}, \ldots, c_{n}\langle$, therefore $e_{\text {base }}$ rolls up to exactly one element in $\} c_{1}, \ldots, c_{n}\left\langle\right.$ (let's call it $e_{m i d}$ ). Again, since $\operatorname{DMSet}_{g}\left(c_{1}\right)=\ldots=\operatorname{DMSet}_{g}\left(c_{n}\right)=\mathcal{C}, e_{\text {mid }}$ has to belong to one of the categories in $\bigcup_{k=1}^{n} g\left(c_{k}\right)$. To prove $e_{m i d}$ is the only element in $\bigcup_{k=1}^{n} g\left(c_{k}\right)$ that has this property, let's assume $e_{\text {base }}$ rolls up to $e_{\text {other }} \vDash e_{\text {mid }}$ and $e_{\text {other }}$ belongs to $\bigcup_{k=1}^{n} g\left(c_{k}\right)$ and rolls up to some element in $c^{\prime}$. Because of the empty data movement set of categories $\} c_{1}, \ldots, c_{n}\langle$ and $c, e_{\text {other }}$ should also belong to $\} c_{1}, \ldots, c_{n}\langle$ and roll up to some element in $c$ which is impossible since $c$ is summarizable from $\} c_{1}, \ldots, c_{n}\left\langle\right.$. Therefore, $c^{\prime}$ is summarizable from $\bigcup_{k=1}^{n} g\left(c_{k}\right)$.

Example 15 For the inconsistent dimension of Figure 1.1, consider the structural repair of Figure 3.2, with the schema mapping

$$
\begin{aligned}
g_{1}: & \left.\left.\operatorname{City} \emptyset^{\top}\right\} \operatorname{City} 1, \operatorname{City} 2, \operatorname{City} 3<, \text { State } \emptyset^{\top}\right\} \text { State }\langle, \\
& \text { Province } \left.\emptyset^{\top}\right\} \text { Province }\left\langle\text {, Country } \emptyset^{\top}\right\} \text { Country1, Country } 2<, \\
& \text { Continent } \left.\emptyset^{\top}\right\} \text { Continent }\left\langle, \text { All } \emptyset^{\top}\right\} \operatorname{All}\langle.
\end{aligned}
$$

Since $g$ has an empty data movement set for every category, we expect it to preserve summarizability properties that hold in the original dimension. For example, Province is summarizable from City in the dimension in Figure 1.1. In the MSR in Figure 3.2, Province is summarizable from $\}$ City1, City2, City3<.

Notice in the previous example that, in addition to the preservation of existing summarizability properties, the structural repair is also adding extra summarizibility properties. For instance, category All was not summarizable from City through category Country in the original dimension, but it is now summarizable from \}City1, City2, City3< through category Country1. This is achieved through the newly imposed strictness and homogeneity conditions.

\subsection{Virtual Repairs in MDX}

An important problem that needs to be addressed is how to implement structural repairs in a way that is transparent to users of MDDBs. As discussed in previous sections, one 
advantage of using structural repairs instead of data repairs is that the former remove inconsistencies without having to change the underlying database.

In the case of a ROLAP data warehouse, we can apply schema modifications by generating database views on top of the existing data structures. Having access to a relational data source, one can create and store database views to model structural repairs. Although this protects data from being modified, it still involves accessing the underlying database to create views (an administrative privilege that may not be assigned to every user).

One of the interesting features of the MDX query language is the ability to define queryscoped calculated members. A query-scoped calculated member is a virtual object defined in terms of other existing objects by means of a query. For example, a new category (or data element) in terms of other existing categories (data elements, resp.), etc. They are generated using the WITH construct in MDX:

\section{WITH \}MEMBERISET〈 MemberIdentifier AS ' member-formula' SELECT …}

This template creates a new calculated member (or set of members) that is defined by the MDX expression 'member-formula'. \}MEMBER ISET/ indicates if the newly created object is an element or a category, resp.

Using this feature, structural repairs can be virtually created, without any need for materializing new data or accessing the database in order to generate database views. This is specially useful if the structural repair of choice is going to be materialized at some point, after some exploratory analysis. As mentioned earlier, multiple SRs may exist for a single inconsistent HM dimension. All of which can be virtually created.

A query that is imposed on the original instance (whether computing results from scratch, i.e. the fact table or using existing pre-computed cube views at lower levels) will be rewritable for MSRs and the rewriting (in all MSRs) will produce the same result as the original query. This is a different behaviour compared to data repairs investigated in [13] where queries on different repairs could possibly produce different results which in turn leads to obtaining consistent query answers (answers that are shared between all repairs). In the case of structural repairs, it is good enough to obtain a single MSR and use that for the derivation of correct query results. 
Example 16 The following MDX query creates a query-scoped calculated category StateProv, as the union of categories State and Province. It then selects the summarized value of measure Sales for the members in this new category. Here, Salescube is the base cube (or fact table):

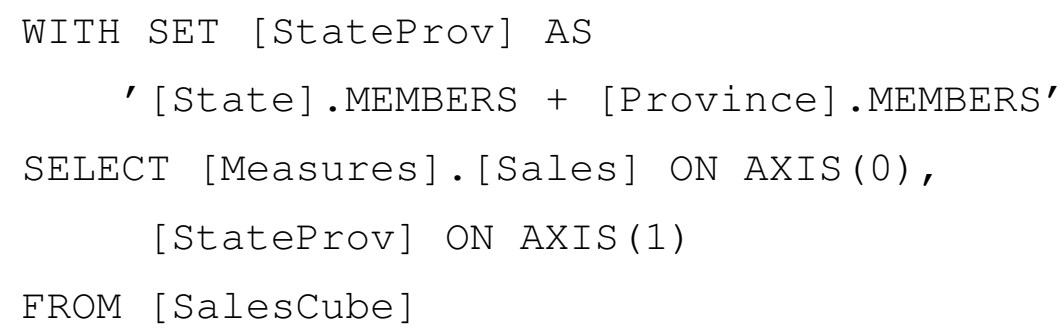

Structural repairs (or portions of them) can be generated using this form of view definitions, as virtual repairs. In them, new categories are defined as calculated members. Virtual repairs can be used to test how structural changes will affect existing queries before making the transition to the new MD model.

Example 17 (example 16 cont.) Consider the structural repair of Figure 3.2. The given MDX query defines StateProv, the new category in the structural repair of Figure 3.1. It contains the elements in the union of categories State and Province.

\subsection{Discussion}

Fixing non-strictness via structural repairs has good properties in terms of the number of changes when the parent elements in a same category involved in a violation of strictness (i.e. they have a descendant in common) do not have descendants in many different categories. In the opposite case, changing the parent category may have to be propagated to categories belonging to its lower subgraph, causing several additional changes, as shown in the next example.

Example 18 Consider the non-strict dimension of Figure 3.4a, where element e2 rolls up to two different elements, e7 and e8, in category C4. Here, e7 and e8 have descendants from three different categories in their subgraph. As shown in Figure 3.4b, a structural repair forces many changes in the dimension schema. Using a data repair or a combination of a data and a structural repair may result in fewer overall changes. 


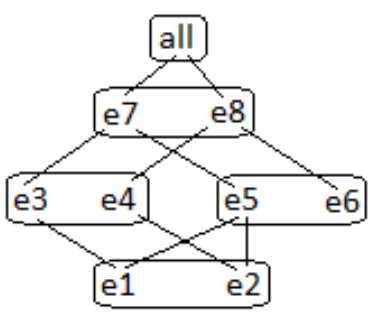

(a) A non-strict dimension

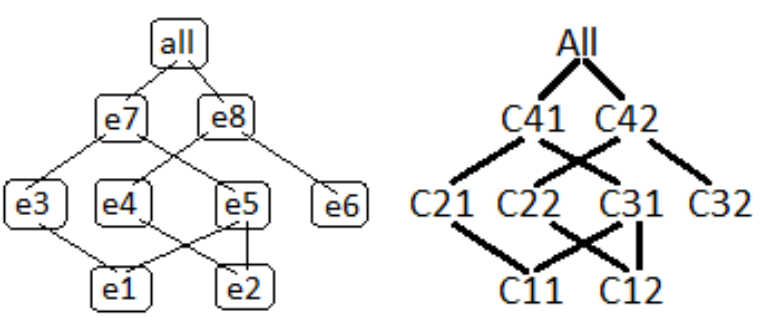

(b) SR causing multiple changes

Figure 3.4: Structural repairs and non-strictness

In cases where resolving non-strictness (at higher levels of the dimension) using structural repairs causes addition of numerous new categories (e.g. resolving non-strictness using SRs in Example 18), data repairs usually produce better results i.e. results that are closer to the original dimension (and cause fewer changes).

An important drawback of structural repairs is that categories of the old schema change or disappear. As a result, they require the rewriting of queries posed to the original MD model into queries in terms of new categories (which may not represent real-world concepts that users are familiar with). For example, the disappearance of categories City and Country from the SRs shown in Figures 3.1 and 3.2 will not only make existing queries on the original inconsistent dimension syntactically invalid, but also new categories (e.g. Country1) will not necessarily represent sensible real-world concepts.

Inconsistencies can be resolved by combining (the ideas behind) structural and data repairs. In some cases, taking advantage of both approaches for resolving different local inconsistencies may produce better results in terms of number of changes. This is illustrated in the next example.

Example 19 Figure 3.5a shows an inconsistent dimension. Restriction to pure data repairs will require applying multiple data and link changes. One way is to add at least five new and different NULL elements and ten new links, to resolve heterogeneity; and also remove the link between e 3 and $e 10$, to resolve non-strictness. On the other hand, using a pure structural repair requires introducing at least four new categories. As can be seen in Figure $3.5 \mathrm{~b}$, a combination of structural and data changes provides a solution that involves fewer changes (adding two categories in the dimension schema and removing a single link in the dimension instance). 

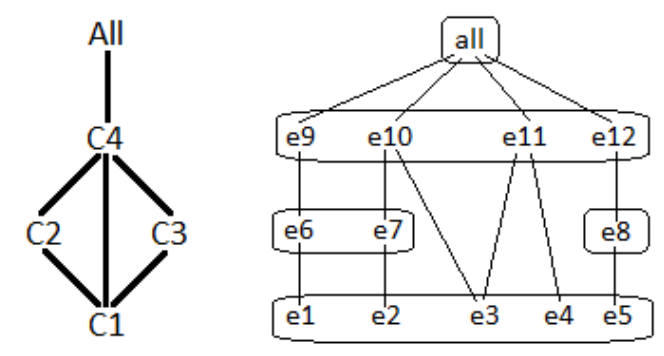

(a) An inconsistent dimension
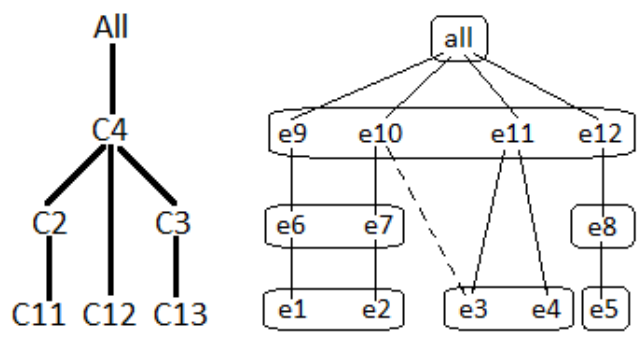

(b) A repair that changes the schema and instance

Figure 3.5: Combining structural repairs and data repairs

To resolve heterogeneity, we can add multiple (different) NULL values as new constants within existing categories. Each element that is missing a parent due to heterogeneity, will be connected to a new NULL. These NULL elements themselves have to be connected to existing elements in parent categories or to other NULL elements depending on the dimension structure. The impact is that the instance will be populated with many NULL elements that have no real-world meaning but will appear in query results (notice that if they are excluded from query results, then those queries cannot be reused for deriving other queries). 


\section{Chapter 4}

\section{The Extended HM Model}

\subsection{The Multidimensional Model}

An EHM dimension schema $\mathfrak{S}$ is a tuple of the form $\backslash U, \mathcal{D}, \mathcal{T}, \Rightarrow, \theta \mid$, where $U$ is a possibly infinite set (the underlying data domain), $\mathcal{D}$ is a finite set of categories (or better, category names), $\mathcal{T}$ is a finite set of subcategories (or names therefor), $\rangle \mathcal{T}, \Rightarrow \mid$ is a directed acyclic graph, and $\theta: \mathcal{T} \nearrow \mathcal{D}$ is a total and onto (surjective) function. $\mathcal{D}$ and $\mathcal{T}$ are disjoint, but when a category has a single subcategory (as determined by $\theta$ ), we will sometimes identify them. Notice that $\Rightarrow$ is a binary relation between subcategories. Its transitive and reflexive closure is denoted by $\Rightarrow^{*}$. We make the additional assumption that any two subcategories assigned to a same category are $\Rightarrow^{*}$-incomparable.

Every dimension schema has a distinguished top category, All, with a single subcategory, also denoted by All, which is reachable from every other subcategory: For every subcategory $s \triangle \mathcal{T}, s \Rightarrow^{*} \mathrm{All}$ holds. For a subcategory $s$, a subcategory $s^{\prime}$ for which $s^{\prime} \Rightarrow s$ $\left(s^{\prime} \Rightarrow^{*} s\right)$ holds is called a child (resp. descendant) of $s$. Subcategories that have no descendants are called base subcategories. The graph structure on $\mathcal{T}$ induces a graph structure on $\mathcal{D}$, which we also denote with $\Rightarrow$ (and $\Rightarrow^{*}$ ): For $c, c^{\prime} \triangle C, c \Rightarrow c^{\prime}$ holds iff there are $s, s^{\prime}$ with $\theta(s)=c, \theta\left(s^{\prime}\right)=c^{\prime}$, and $s \Rightarrow s^{\prime}$. Accordingly, we can talk about categories that are children or descendants of a category. Finally, we also assume that every schema contains a unique base category, i.e. a category with no children. However, this base category may contain one or more base subcategories.

Given a dimension schema $\mathfrak{S}$, a dimension instance for the schema $\mathfrak{S}$ is a tuple $\mathcal{H}=$ \rangle $\mathcal{Q}, \delta,<\mid$, where $\mathcal{Q}$ is the finite subset of $U, \delta: \mathcal{Q} \nearrow \mathcal{T}$ is a total function (assigning data elements to subcategories), and $<$ is a binary relation on $\mathcal{Q}$ that parallels relation $\Rightarrow$ on subcategories: $e_{1}<e_{2}$ iff $\delta\left(e_{1}\right) \Rightarrow \delta\left(e_{2}\right)$. If $\theta(\delta(e))=c$, then we also say by extension that $e \triangle c$. Element all $\triangle \mathcal{Q}$ is the only element of subcategory All.

The transitive and reflexive closure of $<$ is denoted with $<^{*}$, and can be used to define 
the roll-up relations for any pair of categories $\left.c_{i}, c_{j}: \mathcal{S}_{c_{i}}^{c_{j}}(\mathcal{H})=\right\}\left(e_{i}, e_{j}\right) \backslash e_{i} \triangle c_{i}, e_{j} \triangle$ $c_{j}$, and $e_{i}<^{*} e_{j}\langle$. Similarly, roll-up relations can be associated to pairs of subcategories or pairs formed by a category and a subcategory (usually of some other category), We also define $\mathcal{S}_{s}^{T}(\mathcal{H})$, where $T$ is a set of subcategories and $s$ is a single subcategory, to be $\bigcup_{s^{\prime} \in T} \mathcal{S}_{s}^{s^{\prime}}(\mathcal{H})$. Notice that in any case where it is not the case that $c_{i}<^{*} c_{j}$, then $\mathcal{S}_{c_{i}}^{c_{j}}(\mathcal{H})$ is the empty relation. When $c_{i}=c_{j}$, it is the identity relation, with elements from the set \}$(e, e) \backslash \delta(e) \triangle \mathcal{T}$ and $\theta(\delta(e))=c_{i}\langle$.

Definition 9 An instance $\mathcal{H}$ for a schema $\mathfrak{S}$ is compliant if, for a pair of subcategories $s_{i}, s_{j} \triangle \mathcal{T}, \mathcal{S}_{s_{i}}^{s_{j}}(\mathcal{H})$ is a total function.

The condition above will make sure that the subcategory hierarchy will reflect the structure of the instance. Notice that the requirement on the roll-up relations is not imposed on pairs of categories.

Example 20 The Location dimension in Figure 1.4 can be modeled through the following schema S:

$$
\begin{aligned}
& \mathcal{L}_{\text {oc }}=\text { \}City, State, Province, Country, Continent, All }<. \\
& \mathcal{T}_{\text {Loc }}=\text { \}ity1, City2, City3, State, Province, Country1, Country2, Continent, } \\
& \mathrm{All}<\text {. } \\
& \Rightarrow=\} \text { (City1, State), (City2, Province), (City3, Country1), } \\
& \text { (City3, Country2), (State, Country1), (Province, Country1), } \\
& \text { (Country1, Continent), (Country2, Continent), (Continent, All)〈. }
\end{aligned}
$$

For example, here, $\theta($ City 1$)=\theta($ City2 $)=$ City $\triangle \mathcal{D}_{\text {oc }}$. Now, for the corresponding dimension instance $\mathcal{H}$, we have:

$$
\begin{aligned}
\mathcal{Q}_{\text {Loc }}= & \} \text { LA, Vancouver, Ottawa, London, California, BC, ON, USA, Canada, } \\
& \text { England, UK, NA, Europe, all }\langle. \\
<= & (\text { Vancouver, BC }),(\text { Ottawa, ON }),(\text { LA, California }),(\text { London, England }), \\
& (\text { London, UK }),(\text { BC, Canada }),(\text { ON , Canada }),(\text { California, USA }), \\
& (\text { Canada, NA }),(\text { USA, NA }),(\text { England, Europe }),(\text { UK, Europe }), \\
& (\text { NA, all }),(\text { Europe, all })<.
\end{aligned}
$$


Here, for example, $\delta(\mathrm{LA})=$ City1. Now, the ancestors in categories Province and Country of all base elements can be obtained via the following roll-up relations:

$$
\begin{aligned}
\mathcal{S}_{\text {City }}^{\text {Province }}(\mathcal{H})= & \}(\text { Vancouver }, \mathrm{BC}),(\text { Ottawa, ON })\langle. \\
\mathcal{S}_{\text {City }}^{\text {Country }}(\mathcal{H})= & \}(\text { Ottawa }, \text { Canada }),(\text { Vancouver }, \text { Canada }),(\text { LA }, \text { USA }), \\
& (\text { London, England }),(\text { London, UK })<.
\end{aligned}
$$

The second one is not a function. However, the subcategory roll-ups are total functions. For example:

$$
\left.\mathcal{S}_{\text {City2 }}^{\text {Country1 }}(\mathcal{H})=\right\}(\text { Ottawa, Canada }),(\text { Vancouver, Canada })\langle.
$$

is a total function from (the extension of) subcategory City2 to subcategory Country1.

Definition 10 An HM dimension schema is an EHM dimension schema $\mathfrak{S}=\rangle U, \mathcal{D}, \mathcal{T}, \Rightarrow$ ,$\theta \mid$, where $\theta$ is a bijective function. An HM instance for an HM schema $\mathfrak{S}$ is an instance in the sense of EHM.

This definition establishes the connection with the (old) HM models [37, 41]. Basically, in an HM dimension, each category has a single subcategory. Notice that an HM instance for its HM schema may not be compliant. However, a compliant HM dimension according to this definition is (isomorphic to) an HM dimension in the sense of [37, 41] that satisfies strictness and homogeneity (as also defined in [37, 41]).

Definition 11 A multidimensional database schema $M S$ is a pair $\rangle D S, F S \mid$ where $D S=$ \} $\mathcal{T}_{1}, \ldots, \mathcal{T}_{n}\langle$ is a set of EHM dimension schemas and $F S$ is a fact table schema of the form \rangle $c_{1}, \ldots, c_{n}, m_{1}, \ldots, m_{r} \mid$ where every $c_{k}$ is a base category from schema $\mathcal{T}_{k}$ and every $m_{r}$ is a numerical measure. Moreover, a multidimensional database instance MI over schema $M S$ is a pair $\rangle D I, F I \mid$ with $D I=\} \mathcal{H}_{1}, \ldots, \mathcal{H}_{n}\left\langle\right.$ where each $\mathcal{H}_{i}$ is a dimension instance over schema $\mathcal{T}_{i}$ and $F I$, the fact table instance, is a partial function that maps elements $\left(e_{1}, \ldots, e_{n}\right)$ with $e_{k} \triangle c_{k}$ to elements in $\operatorname{dom}\left(m_{1}\right) \oplus \ldots \oplus \operatorname{dom}\left(m_{r}\right)$.

In this thesis, we will be using the terms instance and dimension indistinctly. 


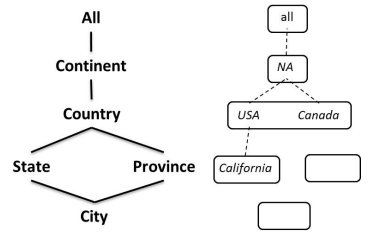

(a)

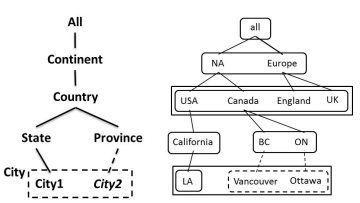

(d)

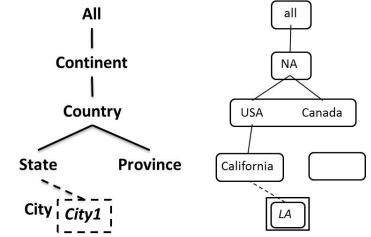

(b)

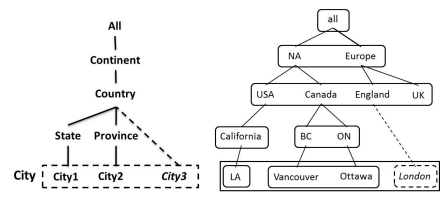

(e)
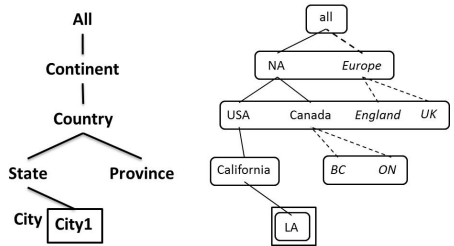

(c)
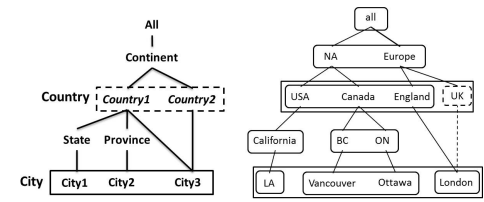

(f)

Figure 4.1: Steps of the add link algorithm for creating the Location dimension

\subsection{Creating EHM Dimensions}

EHM dimensions can be created in one of two ways: either from scratch by inserting links and elements in the dimension schema (discussed next) or from an already existing HM dimension using a repair process (Chapter 7).

In the process of creating an EHM dimension, say a DWH, the user defines and specifies categories (not subcategories). Also, when posing queries, the user thinks in terms of categories. For this reason, subcategories will be defined and maintained by the underlying DWH management system, to keep track of the exact structure of data elements. The existence of subcategories can be transparent to the user.

We propose an incremental algorithm for inserting data links (with already defined parent elements) into a compliant EHM instance. The algorithm guarantees that the instance will remain compliant after the insertion. This will also make it possible to create compliant EHM dimensions from scratch. For this purpose, initially the dimension instance will contain only one element all that belongs to subcategory All. The instance will be populated incrementally by adding links one by one. The algorithm works as described below.

We start with a predefined set of categories in the dimension schema (defined by the DWH user), and with only one subcategory per category. For inserting a link such as $\left(e_{1}, e_{2}\right)$, the parent element (in this case $\left.e_{2}\right)$ should already exist in the instance (this is not 
necessarily the case for the child element). If $e_{1}$ (i.e. the child side of the link) is a new element, the category should be specified as part of the input. One of the following two scenarios will happen:

1. If the new link matches the structure of the subcategory hierarchy (i.e. there is a subcategory $s$ within the category of $e_{1}$ such that if we put $e_{1}$ in $s$, the instance will remain compliant), then we simply add the link and set its subcategory to be $s$. We will call this a compliant addition.

2. If we cannot find such a subcategory, then the subcategory hierarchy must be restructured by adding new subcategories. First, a new subcategory will be added to the category of $e_{1}$. If, by the new addition, the functionality of the subcategory roll-up relations becomes violated, we will also have to add a new subcategory to the category of $e_{2}$ (i.e. the parent). The new subcategory will contain $e_{2}$ as its sole element (notice that we also had the option of moving along with $e_{2}$ any combination of its siblings except the one causing the non-functionality, hence multiple EHM models can be created, but our algorithm chooses only one). In some special cases, multiple categories can be affected. This kind of link addition is called restructuring addition.

A compliant addition is more frequent and less time consuming. But, when we start populating a dimension instance, restructuring additions may occur until the subcategory hierarchy finds its final form (one that matches the external reality). We will first demonstrate these steps with an example.

Example 21 Consider the process of creating the EHM instance of Figure 1.4 from scratch. We can assume the initial schema shown in Figure 4.1 a has been provided as input (by the DWH user, with an instance containing only element, all).

Inserting (NA, all), (USA, NA), (Canada, NA) and (California, USA) is consistent with the initial subcategory hierarchy. Therefore, these are compliant additions and no change in the schema is required. The result of inserting these links is shown in Figure 4.1a. Now, when adding link (LA, California), LA will have no parent in subcategory Province and there is no subcategory in category City that matches this element. Therefore, we will have to create a new subcategory, City1, in category City. Notice we may optionally remove 
the old subcategory City from category City, because it has no elements (but we do not have to). The result of this step is shown in Figure 4.1b.

Next, we may add links (England, Europe), (UK, Europe), (BC, Canada) and (ON, Canada), all consistent with the subcategory hierarchy. So, without any change in the schema, the resulting instance is shown in Figure 4.1c.

The insertion of links (Vancouver, BC) and (Ottawa, ON) will require another restructuring of the subcategory hierarchy. In this case, as shown in Figure 4.1d, another subcategory, City2, must be added to category City that will reflect the structure of new elements being added. Similarly, adding link (London, England) is a restructuring addition because no subcategory of City is connected only to Country (and not to Province or State). As shown in Figure 4.1e, City3 will be added to category City.

Finally, when adding link (London, UK), the roll-up relation between subcategories City3 and Country becomes a non-function, and as a result, we must split category Country into two subcategories, i.e. Country1 and Country2, so that the instance becomes compliant again. The final result is shown in Figure 4.1f.

Algorithm 0 is the precise specification of the add link algorithm described above. We are making the following assumptions in the algorithm:

1. The number of categories, subcategories and links in a dimension schema is very small compared to the size of the dimension instance and can be considered as constant size. Although, theoretically this can be countered, in practice this is a valid assumption.

2. Subcategory $s$ is a matching subcategory for element $e$, iff for every element $e^{\prime}, e<e^{\prime}$ implies $s \Rightarrow \delta_{\mathcal{D}}\left(e^{\prime}\right)$. We assume that finding or creating a matching subcategory for a given element can be done in constant time compared to the size of the dimension instance.

3. Subcategory $s$ is a first common ancestor (or $F C A$ ) of subcategories $s_{1}$ and $s_{2}$, if $s_{1} \Rightarrow^{*} s$ and $s_{2} \Rightarrow^{*} s$ and for no other FCA of $s_{1}$ and $s_{2}$ such as $s^{\prime}, s \Rightarrow^{*} s^{\prime}$. Similarly, subcategory $s$ is a first common descendant (or FCD) of subcategories $s_{1}$ and $s_{2}$, if $s \Rightarrow^{*} s_{1}$ and $s \Rightarrow^{*} s_{2}$ and for no other FCD of $s_{1}$ and $s_{2}$ such as $s^{\prime}, s^{\prime} \Rightarrow^{*} s$. In Figure 1.4, 
subcategory Continent is an FCA and City3 is an FCD for subcategories Country1 and Country2. Whenever a link is being added between subcategories $s_{1}$ and $s_{2}$, the algorithm must check if the roll-ups between these subcategories and their FCAs and FCDs are still functional. If not, the parent side of the non-functional roll-up is going to be split into two subcategories to make it functional (this is done in the Shift-Elements algorithm).

4. The top-conditioned roll-up $\mathcal{S}_{s_{i}}^{s_{j}[E]}$, where $s_{1}$ and $s_{2}$ are subcategories with $s_{1} \Rightarrow{ }^{*} s_{2}$, and $E$ is a set of elements belonging to subcategory $s_{2}$, is the set of all elements from $s_{1}$ that roll up to some element from $E$. More formally, $\left.\mathcal{S}_{s_{i}}^{s_{j}[E]}=\right\} e_{i} \backslash \delta_{\mathcal{D}}\left(e_{i}\right)=s_{i}$ and there exists $e_{j} \triangle E$ with $\delta_{\mathcal{D}}\left(e_{j}\right)=s_{j}$ and $e_{i}<^{*} e_{j}\langle$. Similarly, the baseconditioned roll-up $\mathcal{S}_{s_{i}[E]}^{s_{j}}$ is the set $\} e_{j} \backslash \delta_{\mathcal{D}}\left(e_{j}\right)=s_{j}$ and there exists $e_{i} \triangle E$ with $\delta_{\mathcal{D}}\left(e_{i}\right)=s_{i}$ and $e_{i}<^{*} e_{j}\langle$. In Section 4.3, we present a simple ROLAP implementation of the EHM model and show that computing roll-up relations and conditioned roll-ups can be done using simple relational queries.

5. We assume that moving a set of elements altogether from one subcategory to another can be efficiently done. With our ROLAP implementation of EHM in Section 4.3, this operation can be performed using a batch column update.

\subsubsection{The ADD-LINK Algorithm}

The ADD-LINK algorithm takes as input a new data link $\left(e, e_{p}\right)$ where the child element $e$ can be a new or existing data element, but the parent element $e_{p}$ already exists in the dimension instance. If $e$ is a new data element, adding the link cannot violate semantic constraints, i.e. subcategory roll-ups will remain total functions if this was the case before adding the data link. In this case, the new link will simply be added and the element $e$ will be added to a matching subcategory.

If $e$ is an already existing element, adding a link can possibly violate functionality. This can be the case when either $e$ already has a link to some element other than $e_{p}$ in the subcategory of $e_{p}$, or when $e$ and $e_{p}$ have an FCA or FCD. In both cases, we have to find ancestor elements in the same subcategory that have a shared descendant (this is the source of 


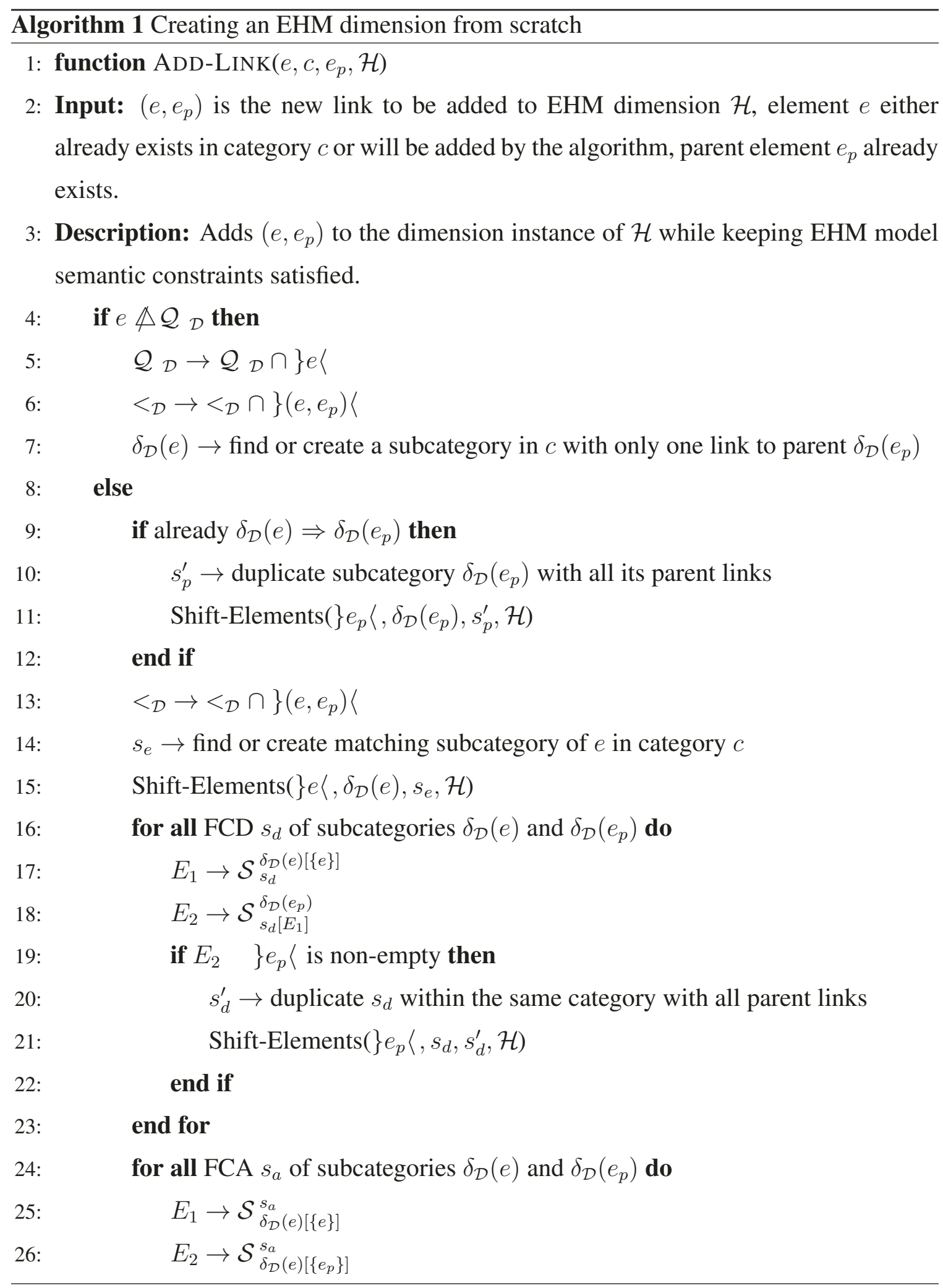


27:

28:

29:

30:

31:

32

\section{3: end function}

34: function $\operatorname{Shift-Elements}\left(E, s, s^{\prime}, \mathcal{H}\right)$

35: Input: $E$ is a set of elements in source subcategory $s, s^{\prime}$ is the destination subcategory, $\mathcal{H}$ is the EHM dimension.

36: Description: Shifts all elements in $E$ from subcategory $s$ to subcategory $s^{\prime}$ and makes necessary adjustments in descendant subcategories to keep EHM model semantic constraints satisfied.

37: $\quad$ Move elements in $E$ from $s$ to $s^{\prime}$ altogether

38: for all subcategory $s_{c}$ with $s_{c} \Rightarrow s$ do

39 :

40:

41:

42:

43:

44:

45: $\quad$ end for

46: $\quad$ if subcategory $s$ is empty then

47: $\quad$ dispose of $s$

48: $\quad$ end if

49: end function 
non-functionality). The subcategory of these ancestor elements must be split into two subcategories and the ancestors should be split between the two to resolve non-functionality. This split operation is done in the Shift-Elements algorithm. When a subcategory is split, we may have to split its child subcategories as well to have subcategory roll-ups that are total functions.

Proposition 3 The execution time of the ADD-LINK algorithm is $O(k \oplus n)$ time where $k$ is the size of the dimension schema (i.e. number of categories) and $n$ is the size of the dimension instance (i.e. number of data elements).

Proof: For compliant additions (which are the most common case) the new link will simply be added with $O(1)$. For restructuring additions, the two for loops between lines 16-31 form the most time consuming part of the algorithm. This is because, in the worst case, the Shift-Elements function can be recursively called for all child subcategories of the source subcategory. The Shift-Elements function, in the worst case, may loop through all elements of each of the child subcategories. Now the Shift-Elements function can be called for all FCDs (usually just one subcategory) and FCAs (again, usually just one subcategory) which in the worst case be $k$ subcategories. Hence, resulting in the worst case execution time of $O(k \oplus n)$ for restructuring additions.

\subsection{EHM and ROLAP schemas}

Multidimensional databases are commonly represented using the star or snowflake relational schemas. In the case of star, a fact table consisting of numerical measurements is directly joined to a set of dimension tables that contain descriptive attributes. On the other hand, the snowflake schema provides a hierarchical relational representation which is the normalized version of star [48].

The EHM model can be implemented by extending these ROLAP schemas and adding tables corresponding to categories that contain more than one subcategory (one column per subcategory). Figure 4.2a shows the representation of the Location dimension as a relational database instance for a star schema. Figure $4.2 \mathrm{~b}$ shows tables that have been added 


\begin{tabular}{c|c|c|c|c|c}
\hline City & State & Province & Country & Continent & All \\
\hline LA & California & null & US & NA & all \\
\hline Vancouver & null & BC & Canada & NA & all \\
\hline Ottawa & null & ON & Canada & NA & all \\
\hline London & null & null & England & Europe & all \\
\hline London & null & null & UK & Europe & all \\
\hline
\end{tabular}

(a) Star representation of Location

\begin{tabular}{c|c|c|c|}
\hline City & City1 & City2 & City3 \\
\hline LA & 1 & 0 & 0 \\
\hline Vancouver & 0 & 1 & 0 \\
\hline Ottawa & 0 & 1 & 0 \\
\hline London & 0 & 0 & 1 \\
\hline
\end{tabular}

\begin{tabular}{|c|c|c|}
\hline Country & Country1 & Country2 \\
\hline US & 1 & 0 \\
\hline Canada & 1 & 0 \\
\hline England & 1 & 0 \\
\hline UK & 0 & 1 \\
\hline
\end{tabular}

(b) Storing EHM subcategories

Figure 4.2: Star implementation of the Location EHM dimension

to store subcategory information for categories City and Country. A similar extension can be applied to the snowflake schema.

With these relational extensions, the computation of roll-up relations between subcategories (e.g. $\mathcal{S}_{\text {City1 }}^{\text {Country1}}$ ) is straightforward and requires only conditional joins with new tables. In addition, adding or deleting subcategories can be performed by simple add column or drop column operations. Data elements can be moved between subcategories using batch column updates. 


\section{Chapter 5}

\section{EHM and Summarizability}

\subsection{Flexible Summarizability for EHM}

The definition of strict-summarizability specified in Section 2.3 can be applied to EHM dimensions as well as HM dimensions. However, in this section, we are going to define a new notion that is more flexible and will cover a wider range of useful models and instances.

In the following, except when otherwise stated, we will consider single EHM dimensions. That is, we consider a dimension schema $\mathfrak{S}=\langle U, \mathcal{D}, \mathcal{T}, \Rightarrow, \theta|$, and a compliant instance $\mathcal{H}=\rangle \mathcal{Q}, \delta,<\mid$. Now we give a definition of summarizability for the extended HM metamodel. However, the new notion of summarizability can be applied to HM dimensions as well as EHM dimensions that may or may not be compliant. Cube views for EHM dimensions can be defined as in Section 2.3.

Definition 12 (a) Category $c$ is $\mathcal{M}$ summarizable with regard to distributive aggregate function $f$, where $\mathcal{M}=\} c_{1}, \ldots, c_{n}\langle$ contains descendants of $c$, iff every cube view on $c$ can be computed by applying $f$ to pre-computed cube views on $\} c_{1}, \ldots, c_{n}\langle$ (and by means of rollup relations between categories and subcategories).

(b) $\mathcal{M}$ s a minimal summarizability set for $c$ iff $c$ is $\mathcal{M}$ summarizable, and there is no $\mathcal{M} \varsubsetneqq \mathcal{M}$ such that $c$ is $\mathcal{M}$-summarizable.

(c) For any dimension $\mathcal{H}$, the summarizability sets of a category c, denoted $\operatorname{SumSets}_{\mathcal{D}}(c)$, is a set containing all minimal summarizability sets of $c$.

(d) Dimension $\mathcal{H}$ is summarizable iff every category $c$ in $\mathcal{D}$ is $\mathcal{M}$ Asummarizable for some subset $\mathcal{M}$ of its child categories. More precisely, for every category $c$ there exists a set $\mathcal{M} \triangle$ SumSets $_{\mathcal{D}}(c)$, such that $\left.\mathcal{M} \leq\right\} c^{\prime} \backslash c^{\prime} \Rightarrow c<$.

Notice that in Definition 12, we are not making any reference to subcategories. However, in a compliant EHM dimension, subcategories (and more specifically their roll-ups) 
will help in the computation of cube views from lower level precomputed cube views. This is demonstrated by the following example.

Example 22 We will demonstrate the effect of subcategories by comparing the HM dimension of Figure 1.1 with the EHM dimension of Figure 1.4. Notice that in the HM dimension, a cube view on category Continent cannot be computed using a precomputed cube view on Country (because element London will be counted twice once through England and another time through UK).

Now, in the case of the EHM dimension of Figure 1.4, a cube view on category Continent can be derived from a precomputed cube view on Country by simply joining with the rollup from subcategory Country 1 as follows:

$$
\Psi_{f}(\text { Continent })=\Psi_{f}(\text { Country }) \bowtie \mathcal{S}_{\text {Country1 }}^{\text {Continent }} .
$$

For any dimension, having access to the summarizability sets of categories can be very useful. Suppose, we are given an aggregate query on category $c$ and we want to compute the result using available pre-computed cube views on descendants of $c$. The class of summarizability sets of $c$ is exactly what we need in order to choose the right set of precomputed cube views to answer the original query. Therefore, being able to compute and generate these sets can have a great impact on improving query answering performance (as long as the process of generating the summarizability sets does not become more complex than answering the query). Once summarizability sets have been computed, they can be used for different queries until some updates on the dimension instance affect and change the summarizability sets.

Example 23 Consider the Sales database of Figure 1.1 plus a Date dimension with categories Day, Month and Year. In this setting, category Country is summarizable from the set of categories \}City, State, Province $\langle$ but the latter is not a minimal summarizability set for Country because Country is also summarizable from $\} \operatorname{City}\langle$. 
The summarizability sets for categories of the Location dimension are as follows:

$$
\begin{aligned}
& \text { SumSets } \left.\left._{\text {Loc }}(\operatorname{All})=\right\}\right\} \operatorname{Continent}\langle,\} \operatorname{Country}\langle,\} \operatorname{City}\langle<, \\
& \text { SumSets } \left.\left._{\text {Loc }}(\text { Continent })=\right\}\right\} \operatorname{City}\langle<, \\
& \text { SumSets } \left.\left._{\text {Loc }}(\text { Country })=\right\}\right\} \operatorname{City}\langle<, \\
& \text { SumSets } \left.\left._{\text {Loc }}(\text { State })=\right\}\right\} \operatorname{City}\langle<, \\
& \text { SumSets } \left.\left._{\text {Loc }}(\text { Province })=\right\}\right\} \operatorname{City}\langle<.
\end{aligned}
$$

Category Continent has only one child, Country, from which it is not summarizable, and therefore, the Location dimension is not summarizable. Actually, we can show that $\Psi_{\text {sum }}$ [Continent, Day] cannot be computed by aggregating over the values of cube view $\Psi_{\text {sum }}$ [Country, Day]. In fact, using the fact table of Figure 1.1c, we obtain the following answers for cube view $\Psi_{\text {sum }}[$ Country, Day]:

$$
\begin{aligned}
& \text { (USA, 1/1/12) } \emptyset^{\top} \text { 0, (Canada, 1/1/12) } \emptyset^{\top} 10500, \\
& \text { (England, 1/1/12) } \emptyset^{\top} \text { 10000, (UK, 1/1/12) } \emptyset^{\top} 10000, \\
& \text { (USA, 1/2/12) } \emptyset^{\top} 3000,\left(\text { Canada, 1/2/12) } \emptyset^{\top} 5500,\right. \\
& \text { (England, 1/2/12) } \emptyset^{\top} 1400,(\mathrm{UK}, 1 / 2 / 12) \emptyset^{\top} 1400 .
\end{aligned}
$$

Combining these results with $\mathcal{S}_{\text {Country }}^{\text {Continent }}$, we will obtain cube view $\Psi_{\text {sum }}$ [Continent, Date] as:

$$
\begin{aligned}
& \text { (NA, 1/1/12) } \emptyset \text { 10500, (Europe, 1/1/12) фr 20000, } \\
& \text { (NA, 1/2/12) ф` 8500, (Europe, 1/2/12) ф 2800, }
\end{aligned}
$$

which is not correct since sales for London have been counted twice for Europe.

Example 24 For the EHM Location dimension of Figure 1.4, the summarizability sets are as follows:

$$
\begin{aligned}
& \text { SumSets } \left.\left._{\text {Loc }}(\mathrm{All})=\right\}\right\} \operatorname{Continent}\langle,\} \operatorname{Country}\langle,\} \operatorname{City}\langle<, \\
& \text { SumSets } \left.\left._{\text {Loc }}(\text { Continent })=\right\}\right\} \operatorname{Country}\langle,\} \operatorname{City}\langle<, \\
& \text { SumSets } \left.\left._{\text {Loc }}(\text { Country })=\right\}\right\} \operatorname{City}\langle<, \\
& \text { SumSets } \left.\left._{\text {Loc }}(\text { State })=\right\}\right\} \operatorname{City}\langle<, \\
& \text { SumSets } \left.\left._{\text {Loc }}(\text { Province })=\right\}\right\} \operatorname{City}\langle<.
\end{aligned}
$$


As shown above, the summarizability sets of each category contain at least one set that is fully composed of its direct children. Therefore, this dimension is summarizable.

In Proposition 4 below, we characterize the notion of summarizability in terms of rollup relations (i.e. in purely algebraic terms). As shown by Example 22, in a compliant EHM dimension we can take advantage of subcategory roll-ups to summarize over existing cube views and compute values for higher level cube views. This justifies the presence of subcategories in the following proposition.

Proposition 4 An EHM instance $\mathcal{H}$ (compliant or non-compliant) with schema $\mathfrak{S}=\rangle U, \mathcal{D}, \mathcal{T}, \Rightarrow$ ,$\theta \mid$ is summarizable iff, for every subcategory $s \triangle \mathcal{T}$ there exists a set of subcategories $\cup=\} s_{1}, \ldots, s_{n}\left\langle\right.$ with $s_{i} \Rightarrow s$, such that for every base subcategory $b \triangle \mathcal{T}$ :

1. $\operatorname{dom}\left(\mathcal{S}_{b}^{s}(\mathcal{H})\right) \leq \operatorname{dom}\left(\mathcal{S}_{b}^{\mathcal{T}}(\mathcal{H})\right)$, and

2. The relation $\}\left(e_{b}, e_{s}\right) \backslash e_{b} \triangle \operatorname{dom}\left(\mathcal{S}_{b}^{c}(\mathcal{H})\right)$ and $\left(e_{b}, e_{s}\right) \triangle \bigcup_{s \in \mathcal{T}} \mathcal{S}_{b}^{s}(\mathcal{H})\langle$ is a function.

Proof: In [41], the authors show that, for a category $c$ to be summarizable from a set of categories $\mathcal{M}$ every base element that rolls up to some element in $c$ has to pass through one and only one element from categories in $\mathcal{M}$ We can easily modify this condition for the case of EHM models to a subcategory and a set $\mathcal{T}$ of subcategories (instead of set $\mathcal{M}$ of categories). Let's refer to $\mathcal{T}$ as the child selection of $s$. To prove summarizability of a dimension, it is sufficient to find a child selection for every subcategory of that dimension.

We can show that the set of subcategories $U$ as described by Proposition 4 is a child selection for $s$. The first item in Proposition 4 makes sure that every base element that rolls up to an element in subcategory $s$, rolls up also to an element in one of the subcategories in $\cup$. The second condition guaranties that each base element $e_{b} \triangle b$ that rolls up to an element $e_{s} \triangle s$ does that through only one element from subcategories that belong to $U$, otherwise $\mathcal{S}_{b}^{\mathcal{T}}(\mathcal{H})$ would not have been a function.

Remark 1 Proposition 4 can be applied without change to an HM instance. As before, we assume that every category in the schema has a single subcategory. 
Example 25 Consider the HM Location dimension of Figure 1.1. For subcategory $s=$ Continent and base subcategory $b=$ City, we have only one option as the set of child subcategories of Continent, namely $\cup=\}$ Country $\langle$. Now, we have:

$$
\begin{aligned}
\mathcal{S}_{b}^{s}(\text { Loc })= & \}(\text { LA }, N A),(\text { Vancouver }, N A),(\text { Ottawa }, N A), \text { (London, Europe })\langle \\
\mathcal{S}_{b}^{\mathcal{T}}(\text { Loc })= & \}(\text { LA }, \text { USA }),(\text { Vancouver }, \text { Canada }),(\text { Ottawa }, \text { Canada }), \\
& (\text { London, England }),(\text { London, UK })\langle.
\end{aligned}
$$

As a result, $\operatorname{dom}\left(\mathcal{S}_{b}^{c}(\mathrm{Loc})\right) \leq \operatorname{dom}\left(\mathcal{S}_{b}^{\mathcal{T}}\right.$ (Loc) $)$ and the first condition of Proposition 4 is satisfied.

As for the second condition in Proposition 4, we can see that the relation \}(LA, USA), (Vancouver, Canada), (Ottawa, Canada), (London, England), (London, UK) $\langle$ is not a function, therefore this condition is not satisfied.

\subsection{Computing Summarizability Sets in HM}

To efficiently answer an aggregate query using pre-computed cube views, we need to be able to decide which cube views produce correct results for the given query. This decision requires the computation of the summarizability sets for categories involved in the aggregate query. In this section, we will show that, unlike HM models, summarizability sets can be efficiently computed for compliant EHM models.

To compute and generate summarizability sets for arbitrary HM models, both the dimension schema and instance have to be processed. This is because, with the HM model, we cannot say much about properties of the summarizability sets. This is due to the fact that in HM, the schema may not reflect the hierarchy of the dimension instance.

Example 26 Summarizability sets in HM do not necessarily have the property of transitivity. This is illustrated with the dimension shown in Figure 5.1. Here, $\} D, E<\triangle$ $\operatorname{SumSets}(B)$ and $\} B, C\langle\triangle \operatorname{SumSets}(A)$ but it is not the case that $\} D, E, C\langle\triangle \operatorname{SumSets}(A)$.

Actually, to generate the summarizability sets for an arbitrary HM model, we need to process the dimension instance. One way to do this is by checking the conditions of 

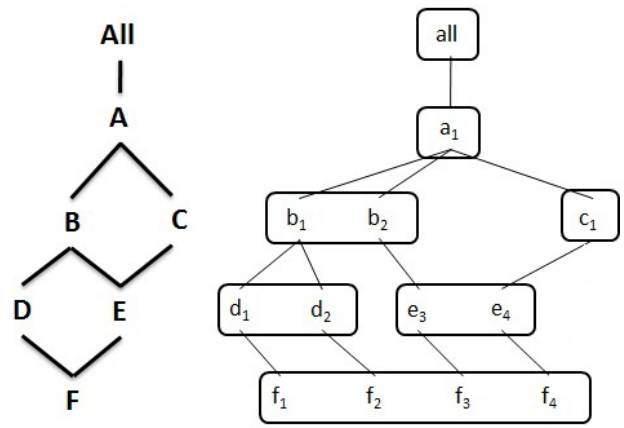

Figure 5.1: An HM dimension with non-transitive summarizability sets

Proposition 4 for every subcategory and every subset of its children (let's call this the naive algorithm).

Proposition 5 The naive algorithm for computing summarizability sets of an HM instance takes $O\left(k \oplus 2^{k} \oplus n \lg n\right)$ time where $k$ is the size of the dimension schema (i.e. number of categories) and $n$ is the size of the dimension instance (i.e. number of data elements).

Proof: The naive algorithm checks the two conditions of Proposition 4 for every category and (in conjunction with) every subset of its children. Now, for the first condition, each roll-up relation can contain up to $O(n)$ elements (these elements can be obtained using a query to the underlying ROLAP implementation). Checking condition 1 in this case can be performed in $O(n \lg n)$. As for the second condition, the relation can contain $O(n)$ elements. Checking if the relation is a function can be done in $O(n \lg n)$. The number of categories is $k$, the number of combinations for subsets of children is $O\left(2^{k}\right)$, therefore, the combined complexity is $O\left(k \oplus 2^{k} \oplus n \lg n\right)$.

The dimension instance is usually very large in size and as a result such a computation becomes impractical. We do not have a practical way to decide whether an aggregation over pre-computed cube views for child categories will produce correct aggregate results for a cube view on a parent category (unless all dimensions are known to be summarizable and remain summarizable after any future updates which is usually not the case). 


\subsection{Computing Summarizability Sets in EHM}

As stated earlier, the hierarchy of subcategories in a compliant EHM dimension reflects the hierarchy of the dimension instance. As a result, we can use this structure (which is smaller in size) to generate the summarizability sets. In the following, we will provide an algorithm that computes summarizability sets for a compliant EHM dimension by only processing its dimension schema (and not the instance itself).

We will first provide a characterization of local summarizability solely expressed in terms of elements of the dimension schema (Proposition 6). This characterization will be based on the notion of base-containment vectors defined next.

Definition 13 Let $\mathcal{H}$ be a compliant EHM instance with schema $\mathfrak{S}=\rangle U, \mathcal{D}, \mathcal{T}, \Rightarrow, \theta \mid$ and base subcategories $s_{1}, \ldots, s_{m}$. The base-containment vector, bcv : $\left.\mathcal{T} \cap \mathcal{D} \nearrow\right\} 0,1\left\langle^{m}\right.$, is a total function that assigns every subcategory $s \triangle \mathcal{T}$ (and every category $c \triangle \mathcal{D}$ ) to an m-ary boolean relation $\left(v_{1}, \ldots, v_{m}\right)$ where $v_{k}$ is 1 , iff $s_{k} \Rightarrow^{*} s$ (or in the case of a category, iff $\left.\theta\left(s_{k}\right) \Rightarrow^{*} c\right)$, otherwise $v_{k}$ is 0 .

Example 27 Consider the EHM dimension of Figure 1.4. The base-containment vector of category Continent is $(1,1,1)$ because all base subcategories (i.e. City1, City2 and City3) roll up to the Continent subcategory which in turn belongs to the Continent category. Also, for subcategories Country1 and Country2 the $b c v$ value is $(1,1,1)$ and $(0,0,1)$, respectively.

We can define the following operators for base-containment vectors of the same arity:

1. Operator + is the usual add operation for two relations of the same arity. Note that the operands and the result have the same arity, but they are not necessarily boolean (i.e. they can contain items greater than 1). As usual, the extension of + for more than two operands will be denoted by $\sum$.

2. Operator $\bigcirc$ is the boolean add operation for two boolean relations of the same arity. The result will also be a boolean relation. We denote the extension of $\bigcirc$ for more than two operands by $\sum_{\oplus}$. 
3. Operator $\geq$ compares two relations of the same arity and succeeds only if all elements of the first relation are smaller or equal to all elements of the second relation.

In addition, we will use $(1, \ldots, 1)_{m}$ to denote an m-ary relation where all items are 1.

The following characterization of summarizability is a result of Proposition 4 for compliant EHM dimensions.

Proposition 6 Let $\mathcal{H}$ be a compliant EHM instance with schema $\backslash U, \mathcal{D}, \mathcal{T}, \Rightarrow, \theta \mid$. Any category $c \triangle \mathcal{D}$ is $\mathcal{M}$ summarizable where $\mathcal{M}=\} c_{1}, \ldots, c_{n}\left\langle\leq \mathcal{D}_{D}\right.$, iff there exists a set of subcategories $\cup=\} s_{1}, \ldots, s_{m}\left\langle\right.$ with $\theta\left(s_{i}\right) \triangle \mathcal{M}$ such that for every subcategory $s$ of $c$, $b c v(s)=\sum_{s_{i} \in \mathcal{T}_{s}} b c v\left(s_{i}\right)$ where $\left.\cup_{s}=\right\} s_{j} \triangle \cup \backslash s_{j} \Rightarrow^{*} s\langle$.

Proof: A value of 1 in the $k$-th item of a subcategory's bcv vector shows that the subcategory is reachable from the $k$-th base subcategory. Therefore, the inequality condition above, i.e. $b c v(s)=\sum_{s_{i} \in \mathcal{T}} b c v\left(s_{i}\right)$, guaranties that every base subcategory that rolls up to a subcategory in $c$ also rolls up to some subcategory in $\cup$. We can extend this result to elements of these subcategories because of the fact that subcategory roll ups are total functions in a compliant EHM model. Hence, every base element that rolls up to an element in $s$, also rolls up to at least one element in $\cup$, proving that $\operatorname{dom}\left(\mathcal{S}_{b}^{s}(\mathcal{H})\right) \leq \operatorname{dom}\left(\mathcal{S}_{b}^{\mathcal{T}}(\mathcal{H})\right)$ (which is the first condition in Proposition 4).

We also know by definition of a bcv vector that for any subcategory $s, b c v(s) \geq$ $(1, \ldots, 1)_{1 \times m}$, which guaranties that $\sum_{s_{i} \in \mathcal{T}} b c v\left(s_{i}\right) \geq(1, \ldots, 1)_{1 \times m}$. This is equivalent to the second condition in Proposition 4 . The result of the summation operation is a vector in which the $k$-th item counts the number of subcategories within $\cup$ that are reachable from the $k$-th base subcategory. For the roll-up relation $\mathcal{S}_{b}^{\mathcal{T}}(\mathcal{H})$ of Proposition 4 to be a function, it is necessary and sufficient that all items in the aforementioned summation result be less than or equal to 1 .

To summarize, Proposition 6 above guaranties the two conditions of Proposition 4 for all subcategories of a category $c$ and is a necessary and sufficient condition for $c$ to be $\mathcal{A}$ summarizable. 
Example 28 (example 27 continued) The Continent category is summarizable from Country in the EHM dimension of Figure 1.4. This is because if we choose $\cup=$ \}Country1<, for subcategory Continent, we have $b c v($ Continent $)=(1,1,1)$ and $b c v($ Country 1$)=$ $(1,1,1)$ therefore $b c v($ Continent $)=b c v($ Country 1$)$.

\subsubsection{The COMPUTE-SUMSETS Algorithm}

Our algorithm for computing summarizability sets is composed of three simple steps:

1. First, it computes $b c v$ values for base subcategories themselves. Notice that this is straightforward since every base subcategory has only one non-zero item in its $b c v$.

2. Then, in a bottom-up order, $b c v$ values for other subcategories will be computed. Notice that we can compute the $b c v$ for any subcategory, having already computed $b c v$ values for its child subcategories (which explains the bottom-up order).

3. Finally, having all base-containment vectors, we can check Proposition 6 for any category $c$ and all combinations of its lower level categories, to see if the respective lower level categories belong to the summarizability set of $c$.

We will first demonstrate these steps with an example.

Example 29 Consider again the EHM dimension of Figure 1.4.

Step 1: We have three base subcategories for which the $b c v$ values are as follows:

$$
\begin{aligned}
& b c v(\operatorname{City} 1)=(1,0,0), \quad b c v(\text { City } 2)=(0,1,0), \\
& b c v(\operatorname{City} 3)=(0,0,1) .
\end{aligned}
$$

Step 2: For all other subcategories the $b c v$ will be initialized to all 0 elements. In a loop (until every subcategory is processed), we will choose parents of already processed subcategories and add (boolean add) to their $b c v$, the $b c v$ value of the child subcategory. For example, we will choose base subcategory City1 as an already processed subcategory, and find all its direct parents (in this case only subcategory State), and add bcv(City1) to $b c v$ (State), therefore $b c v$ (State) becomes $(0,0,0) \bigcirc(1,0,0)=(1,0,0)$. Notice that for a subcategory that has more than one child, such as Country1, the $b c v$ values of all 
the children will be added gradually in the loop to finally obtain the correct value for $b c v$ (Country1) which is $(1,1,1)$. At the end of this loop, the $b c v$ values for all subcategories have been computed.

Step 3: Finally, for any category such as Country, we find the set of categories that roll up to Country, in this case \}State, Province, City $\langle$ and let $L$ be the set of all possible subsets. In this case, $L$ will contain 7 subsets including $\} \operatorname{City}\langle$, $\}$ State, Province $\langle$ and \}City, State, Province $\langle$. Now, for each subset $K \leq L$, we must try all combinations of subcategories from categories in $K$ and check if the condition in Proposition 6 is satisfied. If so, the corresponding set of categories will be added to the summarizability set of Country.

For example, $K=\} \operatorname{City}\langle$ is a subset of $L$. To guaranty satisfaction of requirements in Proposition 6, we must find a subset $T$ of subcategories from $K$ such that for every subcategory $s$ of Country, $b c v(s)=\sum_{s_{i} \in T_{s}} b c v\left(s_{i}\right)$ where $T_{s}$ will be any subcategory of $T$ that rolls up to $s$. For Country 1 with $b c v$ (Country1) $=(1,1,1)$, if we choose the subset $T=$ \}City1, City2, City3 $\langle$ of subcategories from City, we have bcv(City1) $+b c v($ City 2$)+b c v($ City3 $)=(1,1,1)$ which satisfies the condition. For Country2 with $b c v$ (Country2) $=(0,0,1)$, only City3 from $\cup$ rolls up to Country2 and we have $b c v($ City 3$)=(0,0,1)$ which again satisfies the condition. So, we can add category City to the summarizability set of Country.

On the other hand, if we had chosen $K=$ \}State, Province $/$, then no subset of subcategories would have satisfied the equality of Proposition 6 for subcategory Country1, for example $b c v($ State $)+b c v$ (Province) $=(1,1,0)$ which is not equal to $b c v$ (Country1) $=(1,1,1)$. Also, notice that $K=\}$ City, State, Province $\langle$ will not be added to the summarizability set of Country regardless of the condition, because some subset of this (in this case $\} \operatorname{City}\langle$ ) has already been added to the summarizability set of Country.

Proposition 7 Algorithm Compute-SumSets for computing summarizability sets for all categories of a compliant EHM dimension takes $O\left(k \oplus 2^{k}\right)$ time where $k$ is the size of the dimension schema (i.e. number of subcategories). 


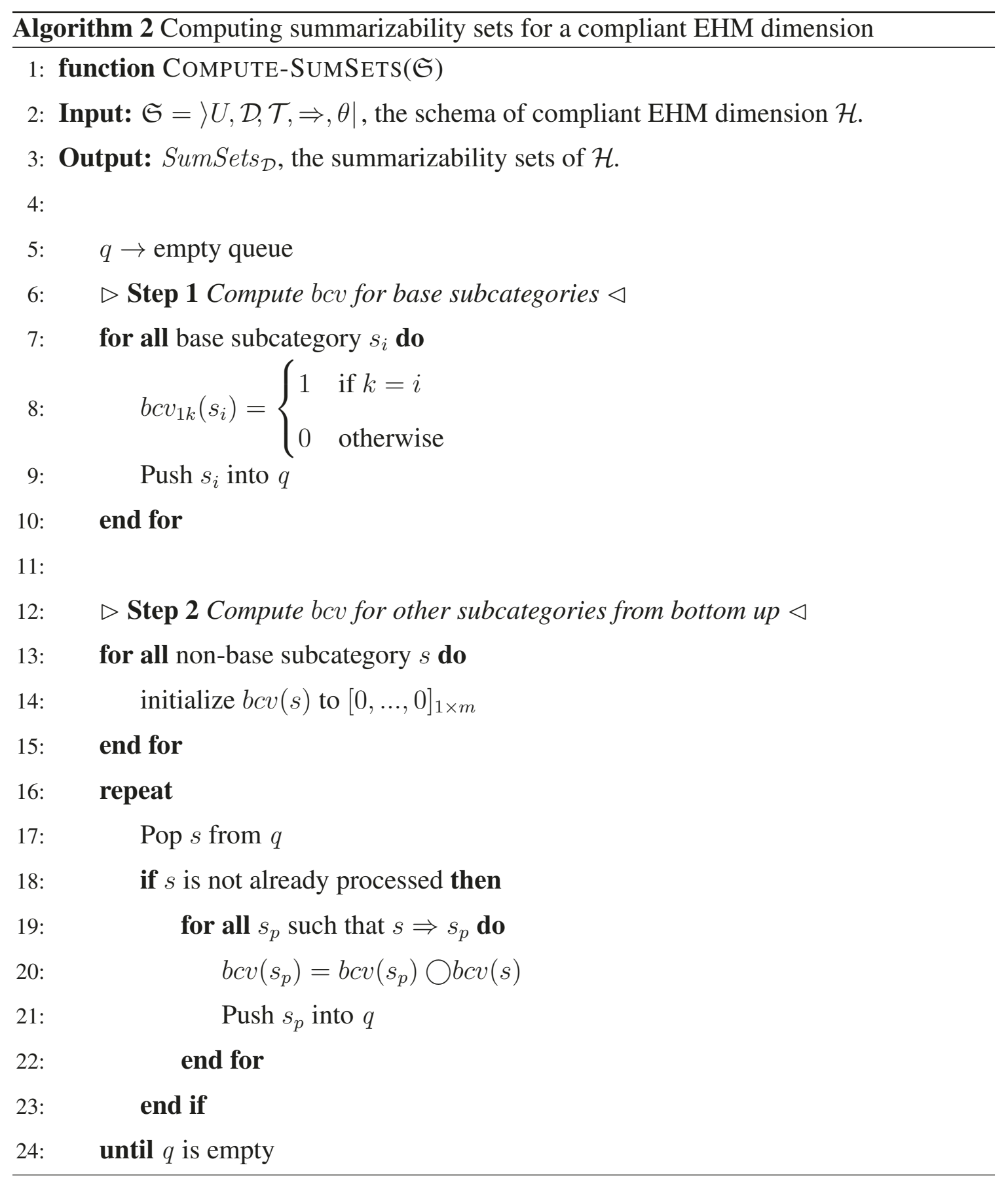




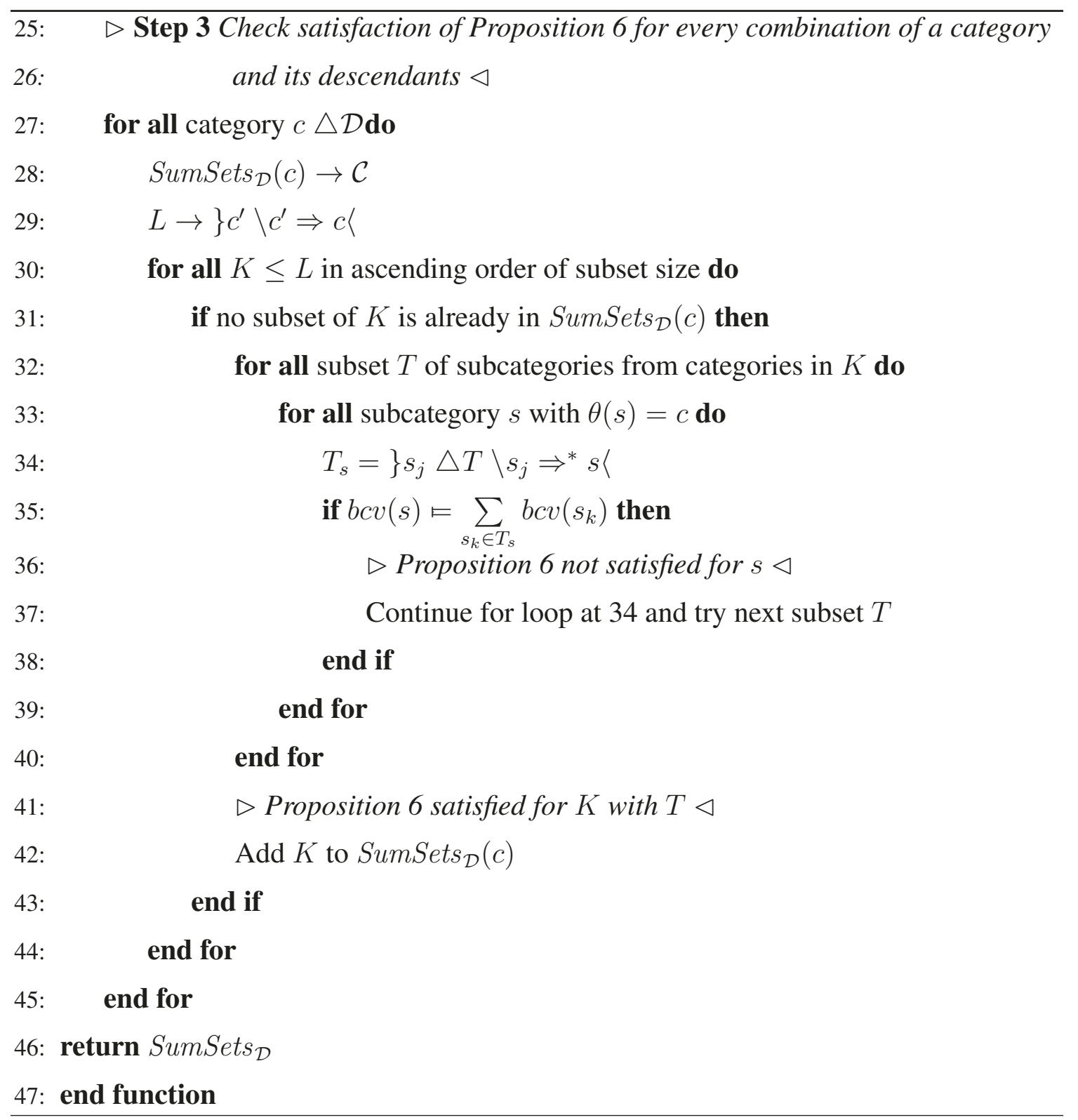


Proof: Step 1 and the first for loop of step 2 iterate over base subcategories and nonbase subcategories (respectively) and initialize their bcv in $O(1)$. Therefore, overall these operations can be done in $O(k)$. The repeat loop of step 2 takes subcategories out of a queue and iterates over all parents of a subcategory and updates the parent's bcv value (so worst case $O(k) \oplus O(1)$ for each subcategory taken out of the queue). Any subcategory will be processed once coming out of the queue (and then it will be marked as processed). Therefore, overall the repeat loop can be done in $O\left(k^{2}\right)$.

The most time consuming step of the Compute-SumSets algorithm is step 3 and specifically statements at lines 34-38 that check the satisfaction of the equality condition in proposition 6. These statements each take $O(k)$ time and are surrounded by 4 for loops. We know that, regardless of the for loops, these statements will be executed only once per any combination of parent subcategory and subset of descendant subcategories. Since we have $k$ subcategories and $2^{k}$ possible combinations of descendants for each one, we can obtain the overall time complexity of $O\left(k^{2} \oplus 2^{k}\right)$.

Corollary 1 Checking if a compliant EHM instance is summarizable can be done in $O\left(k^{2} \oplus\right.$ $2^{k}$ ) time where $k$ is the size of the dimension schema (i.e. number of subcategories).

Proof: This is a direct result from Proposition 7. We can check summarizability by first computing summarizability sets. Then, for each category $c$, we can check if the summarizability sets of $c$ contains at least one set composed of only direct children of $c$, and since the size of any summarizability set is smaller than $2^{k}$, the combined complexity is $O\left(k^{2} \oplus 2^{k}\right)$.

\subsection{EHM and Expressiveness}

A natural question to ask is whether every HM dimension can be replaced by an equivalent compliant EHM dimension, i.e. one with the same elements and links in the dimension instance. We argue that the dimension instance is the representative (or model) of the outside reality being modeled. Therefore, expressiveness can be measured by the range of dimension instances a metamodel (such as HM or EHM) can capture. This is formalized 
by Definition 14. We first formalize what it means for two dimensions to be isomorphic. Notice that we do not bring the schema into the picture. So, the two dimensions can have different schemas (even more, one schema can be modeled using HM and the other using EHM).

In this section, we will be using the term dimension in a general sense to refer to HM dimensions as well as EHM dimensions that may or may not be compliant. We define the notion of expressiveness for classes of dimensions. For example, we refer to all the dimension instances that can be modeled using a compliant EHM dimension, as the class of compliant EHM dimensions.

Definition 14 (a) EHM dimension instances $\left.\mathcal{H}_{1}=\right\rangle \mathcal{Q}_{1}, \delta_{1},<_{1} \mid$ and $\left.\mathcal{H}_{2}=\right\rangle \mathcal{Q}_{2}, \delta_{2},<_{1} \mid$ (that may or may not be compliant) are isomorphically equivalent, iff there exists bijective function $f: \mathcal{Q}_{1} \nearrow \mathcal{Q}_{2}$ such that for arbitrary data elements $e_{i}, e_{j} \triangle \mathcal{Q}_{1}$, it holds that $e_{i}<_{1} e_{j}$ iff $f\left(e_{i}\right)<_{2} f\left(e_{j}\right)$.

(b) A class of dimensions, $\mathcal{I}_{1}$, expressively covers $\mathcal{I}_{2}$, denoted $\mathcal{I}_{1} \leftarrow \mathcal{I}_{2}$, iff for any $\mathcal{H} \triangle \mathcal{I}_{2}$, there exists $\mathcal{H}^{\prime} \triangle \mathcal{I}_{1}$ such that $\mathcal{H}$ and $\mathcal{H}^{\prime}$ are isomorphically equivalent.

(c) $\mathcal{I}_{1}$ and $\mathcal{I}_{2}$ are expressively equivalent, iff $\mathcal{I}_{1} \leftarrow \mathcal{I}_{2}$ and $\mathcal{I}_{2} \leftarrow \mathcal{I}_{1}$.

(e) $\mathcal{I}_{2}$ is more expressive than $\mathcal{I}_{1}$, iff $\mathcal{I}_{2} \leftarrow \mathcal{I}_{1}$ but not $\mathcal{I}_{1} \leftarrow \mathcal{I}_{2}$.

One can propose having one subcategory per category of an arbitrary HM dimension to produce such a complaint EHM dimension (with the same instance). Unfortunately, the result will not necessarily be a compliant instance. However, in [9] we show that by splitting categories in a correct fashion, we can make roll-up relations become total functions (a process that may result in multiple different base categories). A similar split process can be applied to subcategories of an EHM dimension (instead of categories) to make the instance compliant. The result is that every HM dimension can be replaced by a compliant EHM dimension without changing the dimension instance. This is expressed in Proposition 8.

Proposition 8 The class of compliant EHM dimensions is expressively equivalent to the class of HM dimensions.

Proof: We can show that (in the worst possible scenario), we can always build an EHM model that is isomorphically equivalent to a given HM model, by using the exact same 
dimension instance, the same set of categories, and with a subcategory hierarchy that is the exact mirror of its dimension instance (i.e. in the subcategory hierarchy of this new EHM model, there exists one subcategory for every data element and one link for each data link in the dimension instance that connects the corresponding categories). It can easily be shown that this new dimension is a compliant instance (i.e. subcategory roll-ups are total functions). First of all, every subcategory in the new dimension contains exactly one element, and therefore subcategory roll-ups are obviously total. On the other hand, we know that links in the subcategory hierarchy have been added according to the links that exist between corresponding elements in the dimension instance. Therefore, whenever a subcategory $s_{1}$ rolls up to subcategory $s_{2}$, the single data element that belongs to $s_{1}$ also rolls up to the single element that belongs to $s_{2}$ which proves that subcategory roll-ups are functions.

As discussed before, elements in the summarizability sets of any dimension are key for speeding up OLAP queries. Another natural question is whether all these items are preserved after replacing an HM dimension with its EHM counterpart.

Definition 15 Let $\mathcal{H}$ and $\mathcal{H}^{\prime}$ be isomorphically equivalent instances with schemas $\rangle U, \mathcal{D}, \mathcal{T}, \Rightarrow$ $, \theta \mid$ and $\rangle U^{\prime}, \mathcal{D}, \mathcal{T}, \Rightarrow^{\prime}, \theta^{\prime} \mid$ (i.e. with the same set of categories). We say that $\mathcal{H}^{\prime}$ covers summarizability items of $\mathcal{H}$, denoted $\mathcal{H}^{\prime} \gg_{\text {sum }} \mathcal{H}$, iff for every category $c \triangle \mathcal{D}$, having $\} c_{1}, \ldots, c_{n} \backslash \triangle \operatorname{SumSets}_{\mathcal{D}}(c)$ implies that there exists $\mathcal{M} \triangle \operatorname{SumSets}_{\mathcal{D}^{\prime}}(c)$ such that $\mathcal{M} \leq\} c_{1}, \ldots, c_{n}\langle$.

Intuitively, if $\mathcal{H}^{\prime}$ covers summarizability items of $\mathcal{H}$ it implies that for any category in $\mathcal{H}$ that is summarizable from a set of its descendants, the isomorphic image of that category in $\mathcal{H}^{\prime}$ is summarizable from the set of isomorphic images of the descendants.

We can show that not only summarizability items of HM dimensions can be preserved when replacing them by compliant EHM dimensions, but also in many cases we can add more items in the summarizability sets that did not exist before. This is captured in Proposition 9.

Proposition 9 Let $\mathcal{H}_{H M}$ be an arbitrary $\mathrm{HM}$ dimension with schema $\left.\mathfrak{S}=\right\rangle U, \mathcal{D}, \mathcal{T}, \Rightarrow, \theta \mid$. There exists an isomorphically equivalent compliant EHM dimension $\mathcal{H}_{E H M}$ with schema $\left.\mathfrak{S}^{\prime}=\right\rangle U^{\prime}, \mathcal{D}, \mathcal{T}, \Rightarrow^{\prime}, \theta^{\prime} \mid$ such that $D_{E H M} \gg_{\text {sum }} D_{H M}$. 
Proof: Consider the same approach used in the proof of Proposition 8 for building an EHM dimension, $\mathcal{H}_{E H M}$, with the same set of categories as HM dimension $\mathcal{H}_{H M}$. It remains to show that $\mathcal{M} \triangle \operatorname{SumSets}_{\mathcal{D}_{H M}}(c)$ implies that for some $\mathcal{M} \leq \mathcal{M}$ we have $\mathcal{M} \triangle \operatorname{SumSets}_{\mathcal{D}_{E H M}}(c)$. Lets first consider $\mathcal{M}$ to have all the categories from $\mathcal{M}$ Clearly, data elements of categories in $\mathcal{M}$ are exactly the same as the data elements of categories in $\mathcal{M}$ Therefore, we can be sure that the following condition holds for $\mathcal{H}_{E H M}$ : every base element that rolls up to $c$, rolls up to one and only one element in subcategories that belong to $\mathcal{M}$, which proves that $c$ is $\mathcal{M}$-summarizable. Now, either $\mathcal{M}$ or one of its subsets will be present in $\operatorname{SumSets}_{\mathcal{D}_{E H M}}(c)$ which proves our goal.

Example 30 The EHM Location dimension of Figure 1.4 covers summarizability items of the HM Location dimension of Figure 1.1. The inverse is not true since \}Country $\langle\triangle$ SumSets $_{\text {Loc }}$ (Continent) for the EHM dimension but Continent is not summarizable from Country in the HM dimension.

Now, a more important comparison should be made between the class of summarizable $\mathrm{HM}$ dimensions and the class of summarizable EHM dimensions. This is expressed in Corollary 2 which is a direct result of Proposition 9.

Corollary 2 The class of summarizable EHM dimensions is more expressive than the class of summarizable HM dimensions.

Proof: From Proposition 9, we know that for any summarizable HM dimension, $\mathcal{H}_{H M}$, there exists an isomorphically equivalent EHM dimension, $\mathcal{H}_{E H M}$ with the same set of categories such that $\mathcal{H}_{E H M} \gg_{\text {sum }} \mathcal{H}_{H M}$. The fact that $\mathcal{H}_{H M}$ is summarizable show that every category $c$ in it is $\mathcal{M}$ summarizable from some subset $\mathcal{M}$ of its own children. From Proposition 9, this means that for some $\mathcal{M} \leq \mathcal{M}$ category $c$ is $\mathcal{M}$-summarizable. Obviously, $\mathcal{M}$ will contain only children of $c$. This holds for every arbitrary $c$, which means that $\mathcal{H}_{E H M}$ is summarizable.

We must also show that there are some EHM dimensions that cannot be modeled using isomorphically equivalent summarizable HM dimensions. We can show this by counterexamples. The Location dimension of Figure 1.4 is such an example. One can show that 
for no HM dimension with the same instance and a base category (that contains all the data elements that have no children) the HM Location dimension becomes summarizable. 


\section{Chapter 6}

\section{Query Answering}

\subsection{The Datalog Setting}

With multiple dimensions, effective use of pre-computed cube views becomes a key task for improving query answering performance. As we will show in this section, the subcategory structure of the EHM model allows us to rewrite queries in terms of a preferred subset of cube views and find the answer in a more efficient manner.

We will use Datalog with aggregation (see Section 2.2.3) to represent MD aggregate queries. Datalog is a formal language with clear semantics and at the same time it is simple and more readable than other languages such as relational algebra, SQL and MDX. For any MD database schema, our datalog representation will include the following sets of predicates:

$\subseteq$ Category predicates of the form $C(e)$ (one predicate per category identifying elements of that category),

$\subseteq$ Subcategory predicates of the form $S(e)$ (one predicate per subcategory identifying elements of that subcategory),

$\subseteq$ Parent-child predicates of the form $<\left(e_{1}, e_{2}\right)$ (one predicate per dimension indicating links in the dimension instance) and predicates the transitive closure $<^{\star}\left(e_{1}, e_{2}\right)$ (also one predicate per dimension),

$\subseteq$ Fact predicates of the form $F\left(b_{1}, \ldots, b_{n}, z\right)$ (one predicate per fact table) where $b_{1}, \ldots, b_{n}$ are base elements and $z$ is a measure attribute. Without loss of generality, we will assume that each fact table contains only one measure attribute,

$\subseteq$ For any pair of categories $c_{1}$ and $c_{2}$, we will define a roll-up predicate as $\mathcal{S}_{c_{1}}^{c_{2}}\left(e_{1}, e_{2}\right) \rightarrow$ $C_{1}\left(e_{1}\right), C_{2}\left(e_{2}\right),<^{\star}\left(e_{1}, e_{2}\right)$, 
$\subseteq$ We will also consider a set of built-in predicates such as $=,<$ (not to be confused with the binary parent-child predicate), etc.

Moreover, cube views (and queries) are represented by intensional predicate symbols defined using rules of datalog with aggregation. We will refer to these intentional predicates as view predicates.

\subsection{Aggregate Queries}

Intuitively, the query answering problem can be described as follows: Given an MD aggregate query $q=\Psi_{f}\left[c_{1}, \ldots, c_{n}\right]$ where $c_{i}$ s are categories from different dimensions, and set of pre-computed cube views $V=\} v_{1}, \ldots, v_{m}\left\langle\right.$, find another query $q^{\prime}$ that uses one or more cube views in $V$ and is equivalent to $q$ (i.e. $q$ and $q^{\prime}$ produce the same answer for any given database).

In a general setting, views are used to answer conjunctive queries [54]. In the case of MDDBs, we can restrict our focus to MD aggregate queries of the form specified next.

Definition 16 Let $\rangle D S, F \mid$ be an MD database schema where $D S=\} \mathcal{T}_{1}, \ldots, \mathcal{T}_{n}\langle$ is a set of EHM dimension schemas and $F$ is a fact table schema. An MD aggregate query over categories $\} c_{1}, \ldots, c_{n}\langle$ is a rule of the form

$$
Q(\bar{e}, \operatorname{Agg}(z)) \rightarrow F\left(e_{b_{1}}, \ldots, e_{b_{n}}, z\right), \mathcal{S}_{b_{1}}^{c_{1}}\left(e_{b_{1}}, e_{1}\right), \ldots, \mathcal{S}_{b_{n}}^{c_{n}}\left(e_{b_{n}}, e_{n}\right), D\left(\bar{e}_{D}\right)
$$

where $\left.\bar{e}, \bar{e}_{D} \leq\right\} e_{1}, \ldots, e_{n}\left\langle\right.$, and for any $1 \geq i \geq n, b_{i}$ is the base category of dimension schema $\mathcal{T}_{i}$. Also, $D\left(\bar{e}_{D}\right)$ is a conjunction of literals non of which is a fact predicate or view predicate. We make the additional assumption that $D\left(\bar{e}_{D}\right)$ is not a contradiction (in which case the query would become trivial).

Example 31 Consider the Location and Date EHM dimensions depicted by Figure 6.1, the fact table of Figure 1.1c. An MD aggregate query to obtain sale values in different continents for years after 2005 can be represented as follows:

$$
\begin{aligned}
Q(\text { cnt }, \text { year, Sum }(\text { sales })) \rightarrow & F(\text { cty, day, sales }), \mathcal{S}_{\text {City }}^{\text {Continent }}(\text { cty }, \text { cnt }), \\
& \mathcal{S}_{\text {Day }}^{\text {Year }}(\text { day, year }), \text { year }>2005
\end{aligned}
$$



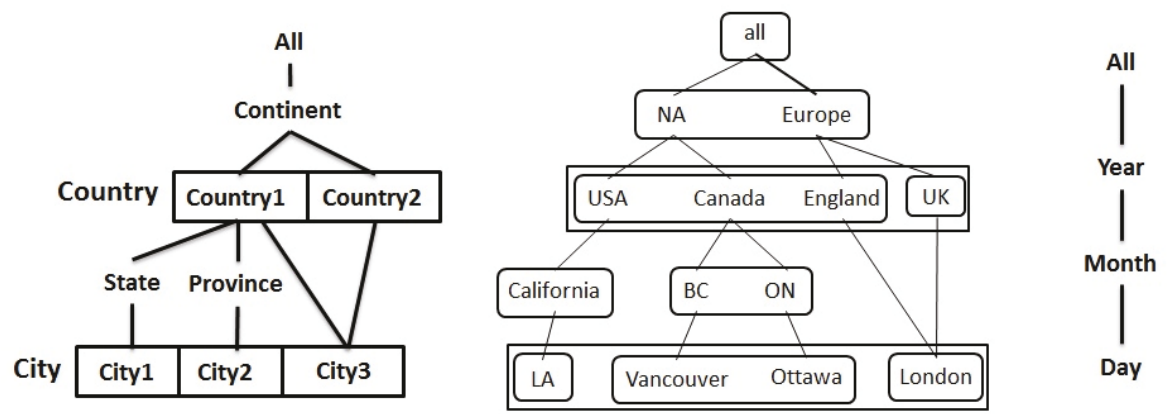

Figure 6.1: The Location and Date EHM dimensions

As stated earlier, we are looking for rewritings that are equivalent to a given aggregate query, but instead of computing results from scratch (i.e. by aggregating over the fact table instance), a rewriting computes results using pre-computed cube views.

Definition 17 Let $V=\} v_{1}, \ldots, v_{m}\langle$ be a set of pre-computed cube views and $Q$ be an MD aggregate query over categories $\} c_{1}, \ldots, c_{n}\left\langle\right.$ as in Equation (6.1). Query $Q_{V}^{\prime}$ is a rewriting for $Q$ over $V$ if:

$\subseteq Q$ and $Q_{V}^{\prime}$ are equivalent (i.e. produce the same answer for any given database), $\subseteq Q_{V}^{\prime}$ is obtained through a set of rules of the following form:

$$
\begin{aligned}
P\left(\bar{e}_{1}, \operatorname{Agg}(z)\right) & \rightarrow V_{1}\left(\bar{e}_{1}^{\prime}, z\right), D_{1}\left(\bar{e}_{D_{1}}\right) \\
\vdots & \\
P\left(\bar{e}_{m}, \operatorname{Agg}(z)\right) & \rightarrow V_{m}\left(\bar{e}_{m}^{\prime}, z\right), D_{m}\left(\bar{e}_{D_{m}}\right) \\
Q_{V}^{\prime}(\bar{e}, \operatorname{Agg}(z)) & \rightarrow P(\bar{e}, z), D\left(\bar{e}_{D}\right)
\end{aligned}
$$

where for any $1 \geq r \geq m, V_{r}$ is a view predicate corresponding to view $v_{r} \triangle V$, and $D_{r}\left(\bar{e}_{D_{r}}\right)$ is a conjunction of literals all of whose variables are among those in $\bar{e}_{r} \cap \bar{e}_{r}^{\prime}$ and $D_{r}$ does not contain any view predicate or fact predicate. We will refer to rules that define predicate $P$ (all rules above except the last one) as the core of the rewriting. We will make the assumption that none of the rules in the core is redundant (i.e. such that removing it would produce an equivalent query for all instances). 
Notice that a rewriting as defined above, is a disjunction of aggregate queries (or a single aggregate query if it uses only one view).

Example 32 (example 31 continued) Consider again the Location and Date EHM dimensions, the fact table of Figure 1.1c, query $Q$ from Example 31 and the following set of pre-computed cube views:

$$
\begin{aligned}
V=\} v_{1} & =\Psi_{\text {sum }}(\text { State }, \text { Month }), v_{2}=\Psi_{\text {sum }}(\text { Province }, \text { Year }) \\
v_{3} & =\Psi_{\text {sum }}(\text { City }, \text { Month }), v_{4}=\Psi_{\text {sum }}(\text { Country }, \text { Day })
\end{aligned}
$$

Query $Q_{1}^{\prime}$ below defines a rewriting for query $Q$ over $\left.V_{1}^{\prime}=\right\} v_{1}, v_{2}, v_{3}\langle$ :

$$
\begin{aligned}
& P(\text { cnt, year, Sum(sales })) \rightarrow V_{1}(\text { stt, mnt, sales }), \mathcal{S}_{\text {State }}^{\text {Continent }}(\text { stt }, \text { cnt }), \\
& \mathcal{S}_{\text {Month }}^{\text {Year }}(\text { mnt, year }) \\
& P(\text { cnt, year, Sum }(\text { sales })) \rightarrow V_{2}(\text { prv, year, sales }), \mathcal{S}_{\text {Province }}^{\text {Contint }}(\text { prv, cnt }) \\
& P(\text { cnt, year, Sum(sales })) \rightarrow V_{3}(\text { cty, mnt, sales }), \mathcal{S}_{\text {City }}^{\text {Continent }}(\text { cty }, \text { cnt }), \\
& \mathcal{S}_{\text {Month }}^{\text {Year }}(m n t, \text { year }), \text { City3(cty) } \\
& Q_{1}^{\prime}(\text { cnt, year, Sum }(\text { sales })) \rightarrow P(\text { cnt, year, sales }), \text { year }>2005
\end{aligned}
$$

Notice in the third rule above, the use of subcategory predicate City 3 to specialize view $v_{3}$ and avoid double counting of elements from subcategories City1 and City2 that have a state or province (and therefore are already accounted for in the first two rules). We can define another rewriting $Q_{2}^{\prime}$ for $Q$ over $\left.V_{2}^{\prime}=\right\} v_{4}\langle$ as follows:

$$
\begin{aligned}
Q_{2}^{\prime}(\text { cnt, year, Sum }(\text { sales })) \rightarrow & V_{4}(\text { ctr }, \text { day, sales }), \mathcal{S}_{\text {Country }}^{\text {Continent }}(c t r, \text { cnt }), \\
& \mathcal{S}_{\text {Day }}^{\text {Year }}(\text { day, year }), \text { Country } 1(c t r)
\end{aligned}
$$

In general, the predicate in the head of the rules defining a query (or a rewriting) may select only a subset of variables included in the view predicate (or the fact predicate) in the body. However, in the rest of this thesis, without loss of generality we will assume that the arity of predicates in the head is the same as the arity of view predicates (or fact predicates) in the body. For example, instead of posing a query that asks for sales for different continents (which omits the attribute referring to the date dimension), we can ask for sales for different continents and category All of the date dimension. 


\subsection{The Query Answering Problem}

Given an MD database schema $\mathcal{T}$ and a set of cube views $V$, we focus on the computational problem of finding a rewriting for an MD aggregate query $Q$ :

$$
\begin{aligned}
\operatorname{MDQA}(Q):=\}\left(\mathcal{T}, V, Q_{V}^{\prime}\right) \backslash \quad & \text { for any instance } \mathcal{K} \text { of } \mathcal{T}, \text { and } \\
& \text { tuple } \bar{t}: \mathcal{K} \models Q[\bar{t}] \in \mathcal{K} \models Q_{V}^{\prime}[\bar{t}]\langle
\end{aligned}
$$

Here, the query is a fixed parameter of the problem, and the variable inputs of the computational problem are the MD database schema $\mathcal{T}$ (with $d$ dimension schemas and at most $k$ categories and subcategories overall) and set $V$ of $m$ cube views. The output of the problem is a rewriting $Q_{V}^{\prime}$ for $Q$ over $V$.

As demonstrated by Example 32, we might have multiple options (in the form of rewritings) to answer a given query. Clearly, we would like to find rewritings that are cheaper to evaluate. The cost of evaluation depends on many factors that differ from one application to another [54].

In this thesis, we consider rewritings that reduce the number of relation tuples involved in the evaluation of the rewritten query. Deriving the final result of a query involves join operations between cube views and respective roll-up relations (see Definition 17). To lower the cost of this derivation, we would like to choose a rewriting that is connected to cube views with fewer tuples (therefore reducing the cost of join to derive the query result).

In theory, the number of cube views reused within a rewriting can be as big as the total number of available views. However, with a large number of re-used views in a single rewriting, the query length becomes unreasonably large. Hence, it is natural to set a constant limit on the number of reused views in a rewriting. In the following definition, this limit is captured by constant $C$.

Definition 18 Let $V$ be a set of pre-computed cube views and $Q$ be an MD aggregate query. A preferred rewriting of core size $C$ is a rewriting of $Q$ over $V^{\prime} \leq V$ such that $\left\langle V^{\prime} \backslash \geq C\right.$ and for any rewriting $Q^{\prime \prime}$ of $Q$ over $V^{\prime \prime} \leq V$ with $\mid V^{\prime \prime} \backslash \geq C$ :

$$
\sum_{v \in V^{\prime}} \operatorname{size}(v) \geq \sum_{v \in V^{\prime \prime}} \operatorname{size}(v)
$$

where size $(v)$ represents the number of tuples in cube view $v$. 
Example 33 (example 32 continued) Lets assume $\mathcal{K}$ is an MD database instance containing sales values for 2000 cities spread across 50 states, 10 provinces and 20 countries over a period of 2 years. We can compare rewritings $Q_{1}^{\prime}$ and $Q_{2}^{\prime}$ from Example 32 as follows:

$$
\begin{aligned}
\sum_{v \in V_{1}^{\prime}} \operatorname{size}(v) & =\operatorname{size}\left(v_{1}\right)+\operatorname{size}\left(v_{2}\right)+\operatorname{size}\left(v_{3}\right) \\
& =(50 \oplus 24)+(10 \oplus 2)+(2000 \oplus 24)=49220 \\
\sum_{v \in V_{2}^{\prime}} \operatorname{size}(v) & =\operatorname{size}\left(v_{4}\right)=20 \oplus 2 \oplus 365=14600
\end{aligned}
$$

Therefore, $Q_{2}^{\prime}$ is preferred over $Q_{1}^{\prime}$.

\subsection{Query answering in EHM}

We will show that with EHM dimensions (unlike HM dimensions), we can find rewritings for a query by only processing the dimension schema (as opposed to the dimension instance). The subcategory structure of a set of compliant EHM dimensions provides an important feature which is to specialize a cube view $v_{i}$ to selected subcategories chosen from categories of $v_{i}$. As an example, considering a cube view that contains sales values of countries in different months for the EHM dimensions of Figure 6.1, we can specialize this cube view to subcategory Country1. This way, tuples that involve element UK will disappear from the result.

Definition 19 Let $Q$ be an MD aggregate query (see Definition 16) over categories $\} c_{1}^{\prime}, \ldots, c_{n}^{\prime}\langle$ and $v=\Psi_{f}\left[c_{1}, \ldots, c_{n}\right]$ be a cube view.

(a) A sub-granularity $g$ of cube view $v$ is a total mapping that assigns a category $c_{i}$ (with $1 \geq i \geq n$ ) to itself or one of its subcategories (i.e. $g\left(c_{i}\right)=x$ implies that either $x=c_{i}$ or $x$ is a subcategory with $\left.\theta(x)=c_{i}\right)$.

(b) The specialization of cube view $v$ to sub-granularity $g$ for query $Q$, denoted $v^{Q}(g)$, is defined as:

$$
P\left(e_{1}^{\prime}, \ldots, e_{n}^{\prime}, f(z)\right) \rightarrow V\left(e_{1}, \ldots, e_{n}, z\right), \mathcal{S}_{c_{1}}^{c_{1}^{\prime}}\left(e_{1}, e_{1}^{\prime}\right), \ldots, \mathcal{S}_{c_{n}}^{c_{n}^{\prime}}\left(e_{n}, e_{n}^{\prime}\right), S\left(\bar{e}_{S}\right)
$$

where $S\left(\bar{e}_{S}\right)$ is a conjunction of subcategory predicates. The conjunction includes $S_{i}\left(e_{i}\right)$ iff $\left(c_{i}, s_{i}\right) \triangle g$. 
Notice that, Equation (6.6) is basically a roll up from categories of view $v$ to categories of query $Q$ with the exception that $S\left(\bar{e}_{S}\right)$ filters only those elements that are part of subcategories of granularity $g$.

Example 34 (example 31 continued) Consider the Location and Date EHM dimensions depicted by Figure 6.1, the fact table of Figure 1.1c and a pre-computed cube view $v=$ $\Psi_{\text {sum }}[$ Country, Month] containing tuples

\}(Canada, Jan, 16000), (England, Jan, 11400), (US, Jan, 3000), (UK, Jan, 11400)<

In this setting:

(a) $\left.g_{1}=\right\}$ Country $\nearrow$ Country1, Month $\nearrow \operatorname{Month}\langle$ is a sub-granularity of $v$ (category Country is assigned to subcategory Country 1 and category Month is assigned to itself). $\left.g_{2}=\right\}$ Country $\nearrow$ Country, Month $\nearrow$ Month $\langle$ is another sub-granularity of $v$ where each category is assigned to itself (there is no filtering).

(b) The specialization of $v$ to $g_{1}$ for query $Q$ (from Example 31 ) is defined by the following rule:

$$
\begin{aligned}
P(\text { cnt }, \text { year }, \text { Sum }(\text { sales })) \rightarrow & V(\text { ctr }, \text { mnt }, \text { sales }), \mathcal{S}_{\text {Country }}^{\text {Continent }}(c t r, \text { cnt }), \ldots, \\
& \mathcal{S}_{\text {Month }}^{\text {Year }}(\text { mnt }, \text { year }), \text { Country } 1(\text { ctr })
\end{aligned}
$$

Here, subcategory predicate Country 1 filters and selects tuples of view predicate $V$ that belong to subcategory Country1.

Definition 20 Let $\rangle D S, F \mid$ be an MD database schema where $D S=\} \mathcal{T}_{1}, \ldots, \mathcal{T}_{n}\langle$ and each $\mathcal{T}_{k}$ is an EHM dimension schema with $m_{k}$ base subcategories, namely $b_{1}^{k}, \ldots, b_{m_{k}}^{k}$. Also, let $v=\Psi_{f}\left[c_{1}, \ldots, c_{n}\right]$ be a cube view and $g$ be a sub-granularity of $v$.

The base containment tensor of sub-granularity $g$, denoted $b c t(g)$, is an $n$-th rank tensor $T=\left(t_{i_{1} \ldots i_{n}}\right)_{m_{1} \times \ldots \times m_{n}}$ with $1 \geq i_{k} \geq m_{k}$ such that:

$$
t_{i_{1} \ldots i_{n}}=\prod_{j=1}^{n} f\left(b_{i_{j}}^{j}, g\left(c_{j}\right)\right)
$$

where $f(b, x)$ assigns a 1 or 0 to base subcategory $b$ and subcategory $x$ depending on whether $x$ is reachable from $b$. If $x$ is a category (as opposed to a subcategory), then 
$f(b, x)$ determines the number of subcategories within $x$ that are reachable from $b$. More precisely,

$$
f(b, x)= \begin{cases}1 & \text { if } x \text { is a subcategory and } b \Rightarrow^{*} x \\ 0 & \text { if } x \text { is a subcategory but not } b \Rightarrow^{*} x \\ \sum_{s \in S_{x}} f(b, s) & \text { if } x \text { is a category containing set of subcategories } S_{x}\end{cases}
$$

Finally, for an MD aggregate query $Q$ over categories $\} c_{1}^{\prime}, \ldots, c_{n}^{\prime}\langle$, we will extend the notion of base containment vector such that $b c t(Q)$ is defined as $b c t\left(g_{Q}\right)$ where $g_{Q}$ assigns every category to itself, i.e. $\left.g_{Q}=\right\}\left(c_{1}^{\prime}, c_{1}^{\prime}\right), \ldots,\left(c_{n}^{\prime}, c_{n}^{\prime}\right)\langle$.

Example 35 (example 34 continued) Consider again the Location dimension with base subcategories $b_{1}^{\text {Loc }}=$ City $1, b_{2}^{\text {Loc }}=$ City2 and $b_{3}^{\text {Loc }}=$ City3, and the Date dimension with base subcategory $b_{1}^{\text {Date }}=$ Day. Also, consider cube view $v$ and sub-granularities $g_{1}$ and $g_{2}$ from Example 34 .

According to Definition 20, $b c t\left(g_{1}\right)$ and $b c t\left(g_{2}\right)$ are $3 \oplus 1$ second rank tensors (i.e. matrices) as follows:

$$
\operatorname{bct}\left(g_{1}\right)=\left[\begin{array}{l}
1 \\
1 \\
1
\end{array}\right] \quad \operatorname{bct}\left(g_{2}\right)=\left[\begin{array}{l}
1 \\
1 \\
2
\end{array}\right]
$$

These matrices can be computed by applying Equation (6.7) to sub-granularities $g_{1}$ and $g_{2}$ to obtain entries of each matrix. For example:

$$
\begin{aligned}
\operatorname{bct}\left(g_{1}\right)_{1,1} & =f\left(\text { City } 1, g_{1}(\text { Country })\right) \oplus f\left(\text { Day, } g_{1}(\text { Month })\right) \\
& =f(\text { City } 1, \text { Country } 1) \oplus f(\text { Day }, \text { Month })=1 \\
\operatorname{bct}\left(g_{2}\right)_{3,1} & =f\left(\text { City3, } g_{2}(\text { Country })\right) \oplus f\left(\text { Day, } g_{2}(\text { Month })\right) \\
& =f(\text { City } 1, \text { Country }) \oplus f(\text { Day, Month }) \\
& =[f(\text { City } 1, \text { Country } 1)+f(\text { City } 1, \text { Country } 2)] \oplus f(\text { Day }, \text { Month }) \\
& =(1+1) \oplus 1=2 .
\end{aligned}
$$

Entry $\operatorname{bct}\left(g_{1}\right)_{1,1}=1$ can be interpreted as follows: the specialization of cube view $v$ to sub-granularity $g_{1}$ for any query will count fact tuples involving base elements from City1 
and Day only once.

On the other hand, entry $b c t\left(g_{2}\right)_{3,1}=2$ is interpreted as follows: the specialization of cube view $v$ to sub-granularity $g_{2}$ for any query will count fact tuples involving base elements from City3 and Day twice. This is because there are two different paths from base subcategory City3 to category Country.

Proposition 10 Let $Q$ be an MD aggregate query over categories $\} c_{1}, \ldots, c_{n}\langle$ and $V=$ \}$v_{1}, \ldots, v_{m}<$ be a set of pre-computed cube views. There exists a rewriting $Q_{V}^{\prime}$ for $Q$ over $V$, iff there exists a set $\} g_{1}, \ldots, g_{m}\left\langle\right.$ where every $g_{k}$ is a sub-granularity of $v_{k}$ such that:

1. $c_{1}, \ldots, c_{n}$ are all reachable from respective subcategories/categories in the range of $g_{k}$ for any $1 \geq k \geq m$, and

2. for every coordinate $i_{1} \ldots i_{n}$, (a) $0 \geq b c t\left(g_{k}\right)_{i_{1} \ldots i_{n}} \geq 1$ and (b) if $b c t(Q)_{i_{1} \ldots i_{n}}>0$ then $\operatorname{bct}\left(g_{k}\right)_{i_{1} \ldots i_{n}}=1$.

Furthermore, if the two conditions above are satisfied, then the set $\} v_{1}^{Q}\left(g_{1}\right), \ldots, v_{m}^{Q}\left(g_{m}\right)\langle$ of specializations will be the core of a rewriting for $Q$.

Proof: Lets assume query $Q$ is defined as follows:

$$
Q\left(e_{1}, \ldots, e_{n}, A g g(z)\right) \rightarrow F\left(e_{b_{1}}, \ldots, e_{b_{n}}, z\right), \mathcal{S}_{b_{1}}^{c_{1}}\left(e_{b_{1}}, e_{1}\right), \ldots, \mathcal{S}_{b_{n}}^{c_{n}}\left(e_{b_{n}}, e_{n}\right), D\left(\bar{e}_{D}\right)
$$

First, we will show that for any set $G=\} g_{1}, \ldots, g_{n}\left\langle\right.$ where $\left.g_{i}=\right\} c_{i 1} \nearrow s_{i 1}, \ldots, c_{i n} \nearrow s_{i n}\langle$ is a sub-granularity of $v_{i}$ and $G$ satisfies the two conditions of Proposition 10, the following set of rules is a rewriting of $Q$ (notice that $\} v_{1}^{Q}\left(g_{1}\right), \ldots, v_{m}^{Q}\left(g_{m}\right)\langle$ is the core of the rewriting):

$$
\begin{aligned}
P\left(e_{1}, \ldots, e_{n}, \operatorname{Agg}(z)\right) \rightarrow & V_{1}\left(e_{11}, \ldots, e_{1 n}, z\right), \mathcal{S}_{c_{1} 1}^{c_{1}}\left(e_{11}, e_{1}\right), \ldots, \mathcal{S}_{c_{1 n}}^{c_{n}}\left(e_{1 n}, e_{n}\right), \\
& S_{11}\left(e_{11}\right), \ldots, S_{1 n}\left(e_{1 n}\right) \\
\vdots & \\
P\left(e_{1}, \ldots, e_{n}, \operatorname{Agg}(z)\right) \rightarrow & V_{m}\left(e_{m 1}, \ldots, e_{m n}, z\right), \mathcal{S}_{c_{m} 1}^{c_{1}}\left(e_{m 1}, e_{1}\right), \ldots, \mathcal{S}_{c_{m n}}^{c_{n}}\left(e_{m n}, e_{n}\right), \\
& S_{m 1}\left(e_{m 1}\right), \ldots, S_{m n}\left(e_{m n}\right) \\
Q_{V}^{\prime}(\bar{e}, \operatorname{Agg}(z)) \rightarrow & P(\bar{e}, z), D\left(\bar{e}_{D}\right)
\end{aligned}
$$


We have to show that any tuple $(\bar{e}, m)$ that is in the result of $Q$ is also in the result of $Q_{V}^{\prime}$ and vice-versa. Lets assume $\left(\bar{e}_{Q}, z_{Q}\right)$ is in the result of $Q$ before applying $D\left(\bar{e}_{D}\right)$. This means that there is a set of tuples $\left.F_{Q}=\right\}\left(\bar{e}_{1 F}, z_{1 F}\right), \ldots,\left(\bar{e}_{r F}, z_{r F}\right)\langle$ from the fact table such that every $\bar{e}_{r F}$ rolls up to $\bar{e}_{Q}$ and $\sum_{i=1}^{r} z_{i F}=z_{Q}$. Now, the core of $Q_{V}^{\prime}$ is selecting all and only tuples $\left.V_{G}=\right\}\left(\bar{e}_{1 G}, z_{1 G}\right), \ldots,\left(\bar{e}_{s G}, z_{s G}\right)<$ that are members of the sub-granularities in $G$. Condition 2 in Proposition 10 guaranties that if $\left(\bar{e}_{F}, z_{F}\right) \triangle F_{Q}$ then $\bar{e}$ will roll up to exactly one tuple $\overline{e_{G}}$ from $V_{G}$ (i.e. with $\left(\bar{e}_{G}, z_{G}\right) \triangle V_{G}$ for some $\left.z_{G}\right)$. Therefore, all tuples from the fact table that are aggregated to generate $\left(\bar{e}_{Q}, z_{Q}\right)$ will be accounted for exactly once within $V_{G}$.

Now, the roll-up predicates in the core of $Q_{V}^{\prime}$ perform an aggregation from tuples in $V_{G}$ to respective ancestors in categories of query $Q$. This roll-up is possible only because of condition 1 from Proposition 10 which guaranties that there exists an upward path to perform such an aggregation. We have shown up to now that any tuple from the fact table that is accounted for in the result of $Q$ before applying $D\left(\bar{e}_{D}\right)$ will also be accounted for exactly once in $V_{G}$ (which is the extension of $P$ in the rewriting). Hence, if we aggregate over $P$ and apply $D\left(\bar{e}_{D}\right)$ to the result, we will obtain a result that is equivalent to the result of $Q$.

Next, we have to prove the other side of Proposition 10, i.e. that a rewriting exists only if there is a set $\} g_{1}, \ldots, g_{n}\langle$ of sub-granularities that satisfies the two conditions of Proposition 10. Lets consider the form of a rewriting as in Definition 17. The core must define the extension of predicate $P$ such that any tuple from the fact table that rolls up to some tuple from the result of $Q$ also rolls up to the extension of $P$. Otherwise the last rule (the aggregation rule that defines $Q_{V}^{\prime}$ from $P$ ) will not yield correct results. For this to happen, $D_{1}\left(\bar{e}_{D_{1}}\right), \ldots, D_{m}\left(\bar{e}_{D_{m}}\right)$ have to be formed as a conjunction of literals that produce the desired extension of $P$ (lets refer to this extension as $\operatorname{Target}_{P}$ ). More precisely, the $m$ rules in the core of a rewriting, generate $m$ partial extensions for $P$, namely $P_{1}, \ldots, P_{m}$ such that for any $i, j, P_{i}\left\{P_{j}=\mathcal{C}\right.$ and $\bigcup_{k=1}^{m} P_{k}=$ Target $_{P}$.

Clearly, the literals in $D_{1}\left(\bar{e}_{D_{1}}\right), \ldots, D_{m}\left(\bar{e}_{D_{m}}\right)$ have to be expressed in terms of categories/subcategories in the schema as opposed to specific elements in dimension instances, because the rewriting must be equivalent to $Q$ for all possible instances of the schema. This means that, to produce each $P_{i}$ from view $v_{i}$ we must find a selection of category/subcategory 
predicates that in conjunction with view predicate $V_{i}$ will filter and produce $P_{i}$. This is exactly what is expressed by condition 1 of Proposition 10. In addition, using links in the schema we have to be able to propagate the results up to the level of categories in query $Q$ which is condition 1 of Proposition 10.

Example 36 (example 35 continued) Consider query $Q$ from Example 31 and sub-granularities $g_{1}$ and $g_{2}$ from Example 35. $g_{1}$ satisfies the two conditions of Proposition 10 because:

1. Continent and Year are reachable from Country1 and Month, and

2. $\operatorname{bct}(Q)=\left[\begin{array}{l}1 \\ 1 \\ 2\end{array}\right]$ and $b c t\left(g_{1}\right)=\left[\begin{array}{l}1 \\ 1 \\ 1\end{array}\right]$, therefore part (a) and (b) of condition 2 are both satisfied for all coordinates of the two matrices.

As a result, specialization $v^{Q}\left(g_{1}\right)$ defined below is the core of a rewriting for $Q$ :

$$
\begin{aligned}
P(\text { cnt, year, Sum }(\text { sales })) \rightarrow & V(\text { ctr, mnt, sales }), \mathcal{S}_{\text {Country }}^{\text {Continent }}(\text { ctr }, \text { cnt }), \\
& \mathcal{S}_{\text {Month }}^{\text {Year }}(\text { mnt }, \text { year }), \text { Country } 1(c t r)
\end{aligned}
$$

However, in the case of sub-granularity $g_{2}, b c t\left(g_{2}\right)_{3,1}=2$, therefore part (a) of condition 2 is not satisfied for $g_{2}$.

The following theorem shows that preferred rewriting can be obtained for an MD database comprised of compliant EHM dimensions without the need to process dimension instances (which are usually very large).

Theorem 5 Let $\rangle D S, F \mid$ be an MD database schema with overall $k$ subcategories, $q$ be an aggregate query, $V$ be a set of $m$ pre-computed cube views and $C$ be a constant. The computational problem of finding a preferred rewriting of core size $C$ for $Q$ is $O\left(m^{C} \oplus\right.$ $\left.2^{k(C+1)}\right)$.

Proof: To obtain a preferred rewriting of core size $C$ for $Q$, we can simply try every combination of sub-granularities over all possible subsets of $V$ with size at most equal to $C$, and for each one check if it satisfies conditions of Proposition 10. Then, between 
all candidates we can compute the sum of cube view cardinalities and select the preferred candidate.

Lets assume $D S$ contains $d$ dimension schemas $\} \mathcal{T}_{1}, \ldots, \mathcal{T}_{d}\langle$ and each dimension schema $\mathcal{T}_{i}$ has $k_{i}$ subcategories. There are $O\left(m^{C}\right)$ possible subsets of $V$ that have a size less than $C$. For each view there are less than $k_{1} \oplus \ldots \oplus k_{d}$ possible sub-granularities (this is because if we have a cube view over categories $c_{1}, \ldots, c_{n}$, then in a sub-granularity each $c_{i}$ can be assigned to either one of the subcategories of $c_{i}$ or to $c_{i}$ itself, and we know that $c_{i}$ has at most $k_{i} \quad 1$ subcategories even in the worst case where it contains all subcategories of the schema except All). So, overall there are $O\left(m^{C} \oplus\left(k_{1} \oplus \ldots \oplus k_{d}\right)^{C}\right)$ possible subgranularities.

Now, for each sub-granularity we need to check the two conditions of Proposition 10. The first condition can be checked in constant time (assuming we have identified and stored in a data structure, the lower level subcategories that categories of each view are reachable from). As for the second condition, for each sub-granularity we need to compute $\left(k_{1} \oplus \ldots \oplus k_{d}\right)$ entries of the base containment tensor. With at most $C$ sub-granularities, the overall complexity becomes $O\left(m^{C} \oplus\left(k_{1} \oplus \ldots \oplus k_{d}\right)^{C} \oplus C \oplus\left(k_{1} \oplus \ldots \oplus k_{d}\right)\right)=$ $O\left(m^{C} \oplus 2^{(C+1) l g\left(k_{1} \times \ldots \times k_{d}\right)}\right)=O\left(m^{C} \oplus 2^{(C+1)\left(l g\left(k_{1}\right)+\ldots+l g\left(k_{d}\right)\right)}\right)<O\left(m^{C} \oplus 2^{k(C+1)}\right)$. 


\section{Chapter 7}

\section{Repairs of Non-Summarizable HM Dimensions}

\subsection{The EHM-based Repair Process}

Summarizability allows for the correct reuse of materialized aggregate views. As discussed in Section 2.3, it is a key property for the efficiency of OLAP systems. Therefore, it is important to address the non-summarizability issue. We do this for the HM model through the EHM models. That is, we define EHM-based repairs of HM instances that are nonsummarizable. In other words, the EHM model allows for a repair approach that transforms non-summarizable HM dimensions into summarizable EHM dimensions. This is an alternative approach to others that directly define and compute repairs for HM instances, e.g. those in $[13,15,19,20]$.

Figure 7.1 sketches the new approach. First, for comparison, with data repairs (left), inconsistency of an HM model is resolved by changing links in the dimension instance without modifying the schema (see Section 2.4). With the EHM-based repairs, we first apply, through what we call canonical mapping, schema-based changes to transform a possibly inconsistent HM instance into a compliant EHM instance (see Section 7.2). As depicted in Figure 7.1, multiple compliant EHM instances may be preferred candidates of this process, one of which will be chosen by a non-deterministic algorithm. Next, if necessary, we apply changes to links in the dimension instance to enforce summarizability, through a process called e-repair (see Section 7.3). We can show that, all e-repairs that cause minimal link changes, i.e. minimal e-repairs, can be found without the need to process the dimension instance for a given compliant EHM dimension.

As we will discuss later, our process will result in more natural solutions for the nonsummarizability problem, and will incur in less computational cost than with data repairs. Notice that, if we start with a non-summarizable EHM model, as opposed to a non-summarizable HM model, we can still apply the same process (with slight modifications to the first step, and excluding it if the input is already compliant) and obtain repairs 


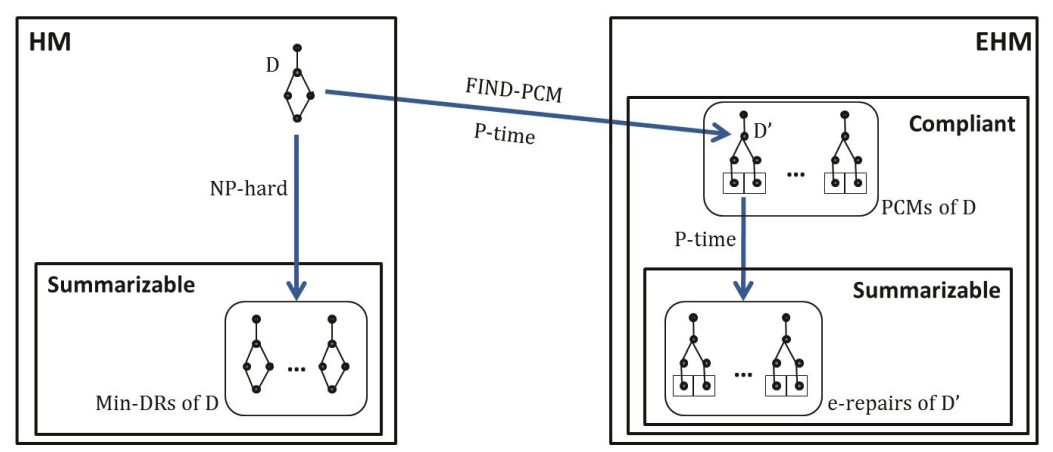

Figure 7.1: Data vs EHM-based repairs (complexities are only in terms of instance size)

of the EHM models.

\subsection{Canonical Mapping: From HM to Compliant EHM Dimensions}

Starting from an inconsistent HM instance, we can find an EHM instance which is compliant, has the same set of elements and links in the dimension instance and preserves all categories in the schema (so that users can refer to those categories in their queries). As discussed in previous sections, a compliant EHM instance has positive computational features compared to an HM instance. Namely, we can check summarizability, compute summarizability sets (Section 5.3) and address the query answering problem (Chapter 6) without processing the dimension instance. In addition, as we will show in Section 7.3, a process similar to the data repair approach can be applied to compliant EHM instances that, compared to data repairs for HM dimensions, results in more natural solutions to the problem and is computationally more practical.

Definition 21 Let $\mathcal{H}=\rangle \mathcal{Q}, \delta,<\mid$ be an (inconsistent) $\mathrm{HM}$ instance over source schema $\mathfrak{S}=\rangle U, \mathcal{D}, \mathcal{T}, \Rightarrow, \theta \mid$. A canonical mapping $(C M)$ for $\mathcal{H}$ is a mapping from $\mathcal{H}$ to a compliant EHM instance $\left.\mathcal{H}^{\prime}=\right\rangle \mathcal{Q}{ }^{\prime}, \delta^{\prime},<^{\prime} \mid$ over target schema $\left.\mathfrak{S}^{\prime}=\right\rangle U, \mathcal{D}, \mathcal{T}, \Rightarrow^{\prime}, \theta^{\prime} \mid$ with the following properties: (a) $\mathcal{Q}=\mathcal{Q}^{\prime},<=<^{\prime}, \mathcal{D}=\mathcal{D}$. (b) For every element $e \triangle \mathcal{Q}$ : $\theta(\delta(e))=\theta^{\prime}\left(\delta^{\prime}(e)\right)$ (i.e. any element belongs to the same category in the source and target schemas).

Example 37 Figures 1.4 and 7.2 show two different canonical mappings for the Location dimension of Figure 1.1. Notice that, links and elements in the dimension instance and 

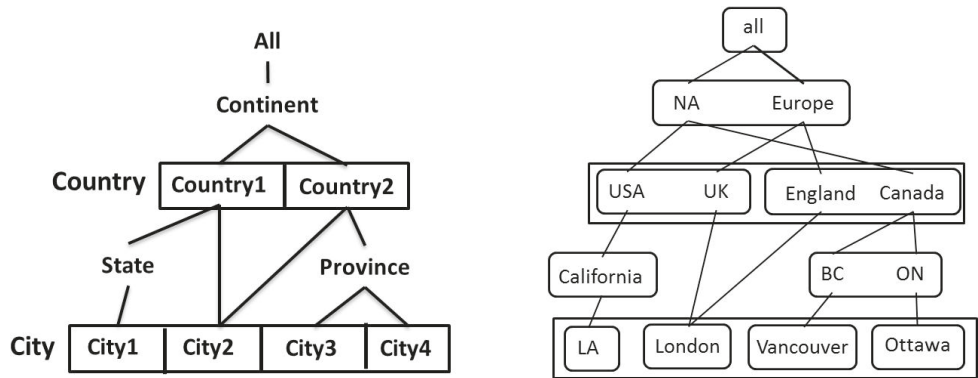

Figure 7.2: A non-summarizable result of a canonical mapping for the Location dimension

categories in the schema remain the same, but new subcategories are introduces (in different ways) to make the EHM instances compliant.

Now, with multiple options for target canonical mapping results, we would like to obtain the ones that redistribute groups of elements as little as possible. For example, the fact that elements Vancouver and Ottawa have been placed in different subcategories in the CM result of Figure 7.2 will usually force queries posed on the City category to require a join to regroup these two elements. This is why in a CM, we will try to limit redistribution of elements as much as as possible.

Definition 22 Let $\mathcal{H}=\rangle \mathcal{Q}, \delta,<\mid$ be an $\mathrm{HM}$ instance and $\left.\mathcal{H}^{\prime}=\right\rangle \mathcal{Q}, \delta^{\prime},<\mid$ be a canonical transformation of $\mathcal{H}$. The redistribution factor of $\mathcal{H}$ and $\mathcal{H}^{\prime}$ is defined by:

$$
\left.\Pi\left(\mathcal{H}, \mathcal{H}^{\prime}\right)=\right\}\left(e_{1}, e_{2}\right) \backslash e_{1}, e_{2} \triangle \mathcal{Q}, \delta\left(e_{1}\right)=\delta\left(e_{2}\right) \text { and } \delta^{\prime}\left(e_{1}\right) \models \delta^{\prime}\left(e_{2}\right)\langle
$$

Intuitively, the redistribution factor is a set containing pairs of elements that were grouped together within the same subcategory in the source HM dimension, but are grouped in different subcategories in the result of the canonical mapping.

Example 38 Let $\mathcal{H}$ be the inconsistent $\mathrm{HM}$ dimension of Figure 1.1, $\mathcal{H}_{1}$ and $\mathcal{H}_{2}$ be results of the canonical mappings of Figures 1.4 and 7.2, respectively. The redistribution factors 
are as follows:

$$
\begin{aligned}
& \left.\Pi\left(\mathcal{H}, \mathcal{H}_{1}\right)=\right\}(\text { LA, Vancouver }),(\text { LA, Ottawa), (LA, London), (Ottawa, London) } \\
& \text { (Vancouver, London), (UK, England), (UK, Canada), (UK, US)< } \\
& \left.\Pi\left(\mathcal{H}, \mathcal{H}_{2}\right)=\right\}(\text { LA, Vancouver }),(\text { LA, Ottawa), (LA, London), (Ottawa, London), } \\
& \text { (Vancouver, London), (Vancouver, Ottawa), (UK, England), } \\
& \text { (UK, Canada), (US, Canada), (US, England) 〈. }
\end{aligned}
$$

Definition 23 A preferred canonical mapping (PCM) of (inconsistent) HM instance $\mathcal{H}$ is a canonical mapping from $\mathcal{H}$ to $\mathcal{H}^{\prime}$ such that $\Pi\left(\mathcal{H}, \mathcal{H}^{\prime}\right)$ is minimal among all canonical mapping results of $\mathcal{H}$.

Example 39 The EHM instance of Figure 1.4 is a PCM of the inconsistent HM instance of Figure 1.1. However, the one in Figure 7.2 is not preferred, because by putting elements Vancouver and Ottawa in the same subcategory we can obtain another CM result for which the redistribution factor is a subset of the former CM.

Proposition 11 There always exists at least one PCM for any HM instance $\mathcal{H}$. If $\mathcal{H}$ is already summarizable, it will be the only PCM of itself.

Proof: Lets consider a compliant EHM instance $\mathcal{H}^{\prime}$ that is isomorphically equivalent with $\mathcal{H}$. From Proposition 9, we know that such a dimension exists. Obviously, $\mathcal{H}^{\prime}$ is the result of a canonical mapping for $\mathcal{H}$ because it has the same set of elements and links in the dimension instance, and the same categories. Also, every element belongs to the same category in both dimensions. Now, either $\mathcal{H}^{\prime}$ is itself a PCM for $\mathcal{H}$ or there exists another $\mathrm{CM}$ that has smaller redistribution factor. In either case, $\mathcal{H}$ has a PCM.

By definition, there might exist more than one possible PCMs for an inconsistent HM dimension. In theory, there might be exponentially many such PCMs. In each PCM, elements of the dimension instance are grouped in a different way in subcategories (while still minimizing the redistribution factor). 


\subsubsection{The FIND-PCM Algorithm}

Algorithm 3, called the FIND-PCM algorithm, computes one of the possible PCMs for a given HM instance. The algorithm is composed of three steps:

1. First, for each element $e$, we will find ancestors of $e$ within any category. For each element, the result will be a mapping from category to set of elements. Any set in the resulting map that contains more than one element indicates a source of inconsistency (because they are different ancestors within a category for one descendant element). So, elements within the set must be put inside separate subcategories in the final result.

2. In the next step, using the result of step one, for each element $e$ we will split other elements of the same category into two disjoint sets: the consistency set $\beta(e)$ and the inconsistency set $\varpi(e)$. $\beta(e)$ will contain all elements that can potentially be in the same subcategory as $e . \varpi(e)$ will contain elements that have at least one shared descendant with $e$, therefore can never be in the same subcategory as $e$.

3. Next, in a top-down order, we will start creating subcategories within each category. Category All will have only one subcategory. Moving down in the category hierarchy, when we reach category $c$, we will take an element from that category, assign it a new subcategory $s_{e}$ and in a greedy fashion, one by one choose $e^{\prime} \triangle \beta(e)$ and add it to $s_{e}$ only if it rolls up to the same subcategories as $e$ (keeping the subcategory roll up a total function). Now that $e$ and $e^{\prime}$ are assigned to the same subcategory, their inconsistency sets must be merged, so remove any $e^{\prime \prime} \triangle \varpi\left(e^{\prime}\right)$ from $\beta(e)$ and add it to $\varpi(e)$. After the iteration is done and no more element remains in $\beta(e)$ to add to $s_{e}$, subcategory $s_{e}$ is finalized. Choose an element from $\varpi(e)$, create a new subcategory and perform a similar iteration with greedy assignment of elements until no more element remains with unassigned subcategory.

We will demonstrate these steps with an example.

Example 40 Consider the HM instance of Figure 1.1.

Step 1: Element London will have no parent in categories State and Province, set \}England, UK $\langle$ of parents in category Country, set $\}$ Europe $\langle$ of parents in category Continent 
and set \}all< of parents in category All. We can find parents of other elements in each category in a similar way.

Step 2: Having the result of step 1, we can compute the consistency set and inconsistency set for each element. For example, elements of the $\}$ Country $<$ category have the following consistency and inconsistency sets:

$$
\begin{array}{ll}
\beta(\mathrm{USA})=\text { \}Canada, England, UK }\langle, & \varpi(\mathrm{USA})=\mathcal{C}, \\
\beta(\text { Canada })=\text { \}USA, England, UK }\langle, & \varpi(\text { Canada })=\mathcal{C}, \\
\beta(\text { England })=\text { \}USA, Canada }\langle, & \varpi(\text { England })=\text { \}UK }\langle, \\
\beta(\mathrm{UK})=\text { \}USA, Canada }\langle, & \varpi(\mathrm{UK})=\text { \}England }\langle.
\end{array}
$$

Step 3: The final step starts with creating a single subcategory within category All that contains element all. Then, in a top-down order, we will move to the next category which is Continent. We will (non-deterministically) take an element from this category, e.g. NA and assign it to a new subcategory, lets call it ContinentS1. Now since $\beta(\mathrm{NA})$ contains Europe we can simply add Europe to subcategory ContinentS1. No element in Continent remains unassigned, so we can move to category Country.

Again, we will start by choosing an element, e.g. USA and creating a subcategory for it, lets call it CountryS1. From $\beta$ (USA) we can choose an element, say England, and since it has the same roll up structure (i.e. it rolls up to the same set of subcategories) add it to CountryS1. Then we have to unify $\beta$ (USA) and $\beta$ (England) causing UK to be removed from $\beta$ (USA). Only one element remains in $\beta(\mathrm{USA})$, namely Canada, which has the same roll up structure and will be added to CountryS1. Next, since no element remains in $\beta$ (USA), we will extract an element from $\varpi$ (USA), which is UK, and create a new subcategory Countrys2 for it. Now, every element of category Country has a subcategory, so we can move to the next category.

Creating subcategories for categories State and Province will be straightforward and will result in one subcategory for each. In case of the City category, as before we will start by choosing an element, say LA and creating a subcategory CityS1 for it. Then, we will choose Vancouver from $\beta(\mathrm{LA})$. However, Vancouver cannot be added to CityS1 since it has a different roll up structure, so it will be moved to $\varpi(L A)$ to be processed later. A similar scenario will occur for Ottawa and London. Hence, LA will be the only 
element of subcategory CityS1. Element Vancouver will be chosen next from $\varpi(L A)$ and we will create a new subcategory for it, namely CityS2. Element Ottawa can be added to this same subcategory, however element London will again require a separate subcategory, namely CityS3 since its roll up structure does not match the one for Vancouver either. No category remains, which indicates the termination of this algorithm.

It is natural to expect the number of ancestors for any element at a single higher level category to be less than a constant upper limit. For example, we can obviously expect each city to roll-up to no more than one continent, or any employee to work under the supervision of no more than two or three managers. In the following proposition, constant $A$ represents such an upper bound.

Proposition 12 Let $\mathcal{H}$ be an inconsistent $\mathrm{HM}$ instance, $k$ be the number of categories, $n$ be the number of elements in the dimension instance and $A$ be a maximum limit on the number of ancestors for any element within a single higher level category. The time complexity of the FIND-PCM algorithm is $O\left(A^{2} k n+n^{3}\right)$.

Proof: The for loop surrounding lines 13-20 will be executed for every element and each of its ancestors, therefore at most $O(A \oplus k \oplus n)$ times overall (because each element has at most $A \oplus k$ ancestors). The inner for loop surrounding lines 24-26 will also run once per each ancestor, however the cost of computing the statement at line 25 is each time $O(A)$, because $P$ may contain up to $A$ elements. Therefore, overall the statement at line 25 will cost $O\left(A^{2} \oplus k \oplus n\right)$ in time complexity. Hence, the overall cost of the for loop surrounding lines 8-29 is $O\left(A^{2} \oplus k \oplus n\right)$.

The for loop surrounding lines 40-47 will be executed only once for each element in the dimension instance. The for loop itself at worst can iterate no more than $n$ times and each iteration can cost $O(n)$ (for removing an element from $E_{c}$ ). So, the overall time complexity of the for loop surrounding lines 30-49 is $O\left(n^{3}\right)$ in the worst case.

Proposition 13 Let $\mathcal{H}$ be an inconsistent $\mathrm{HM}$ instance and $\mathcal{H}^{\prime}$ be a compliant EHM instance. The problem of deciding whether $\mathcal{H}^{\prime}$ is a PCM of $\mathcal{H}$ is P-TIME in the size of the dimension instance. 


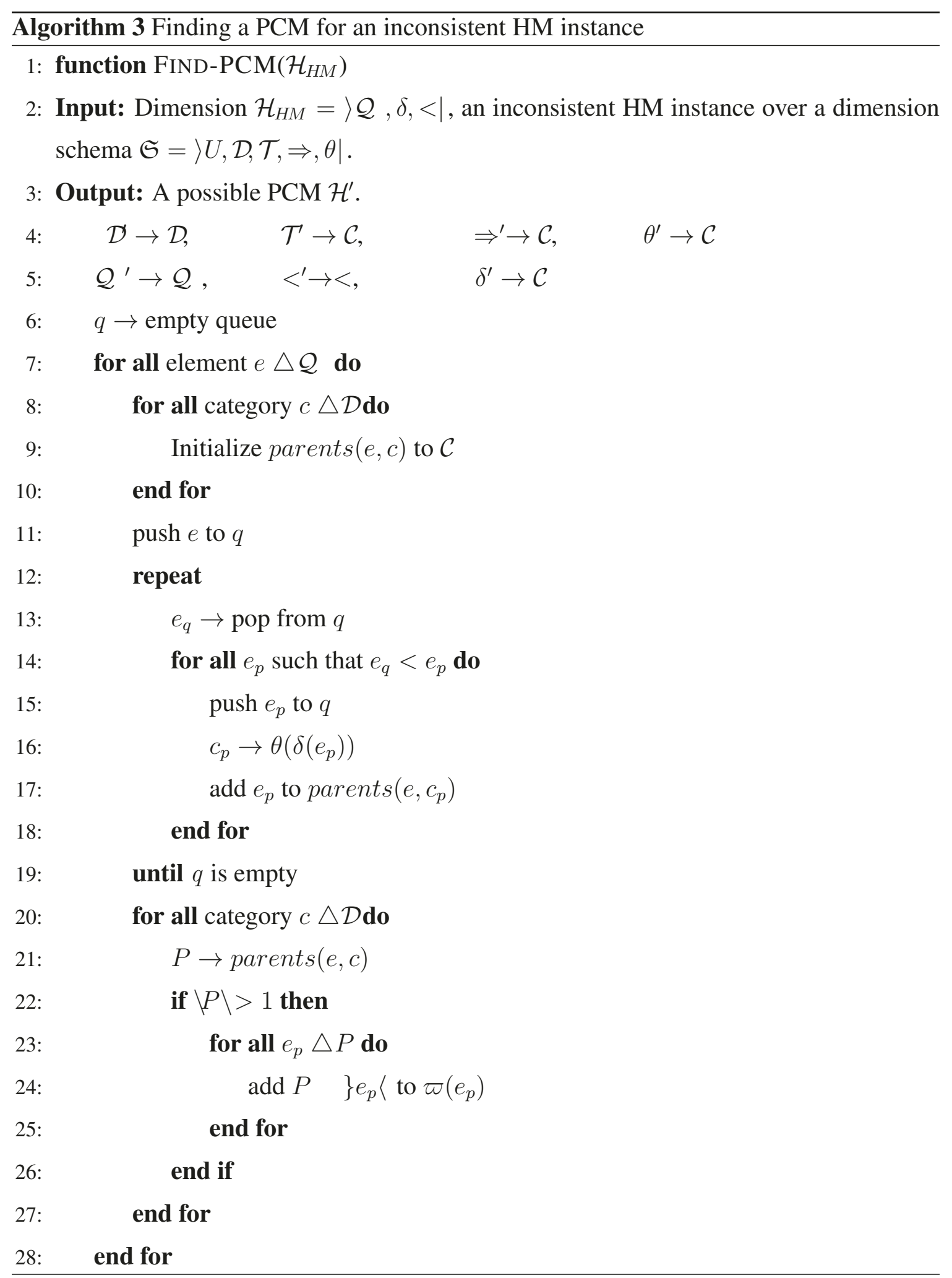




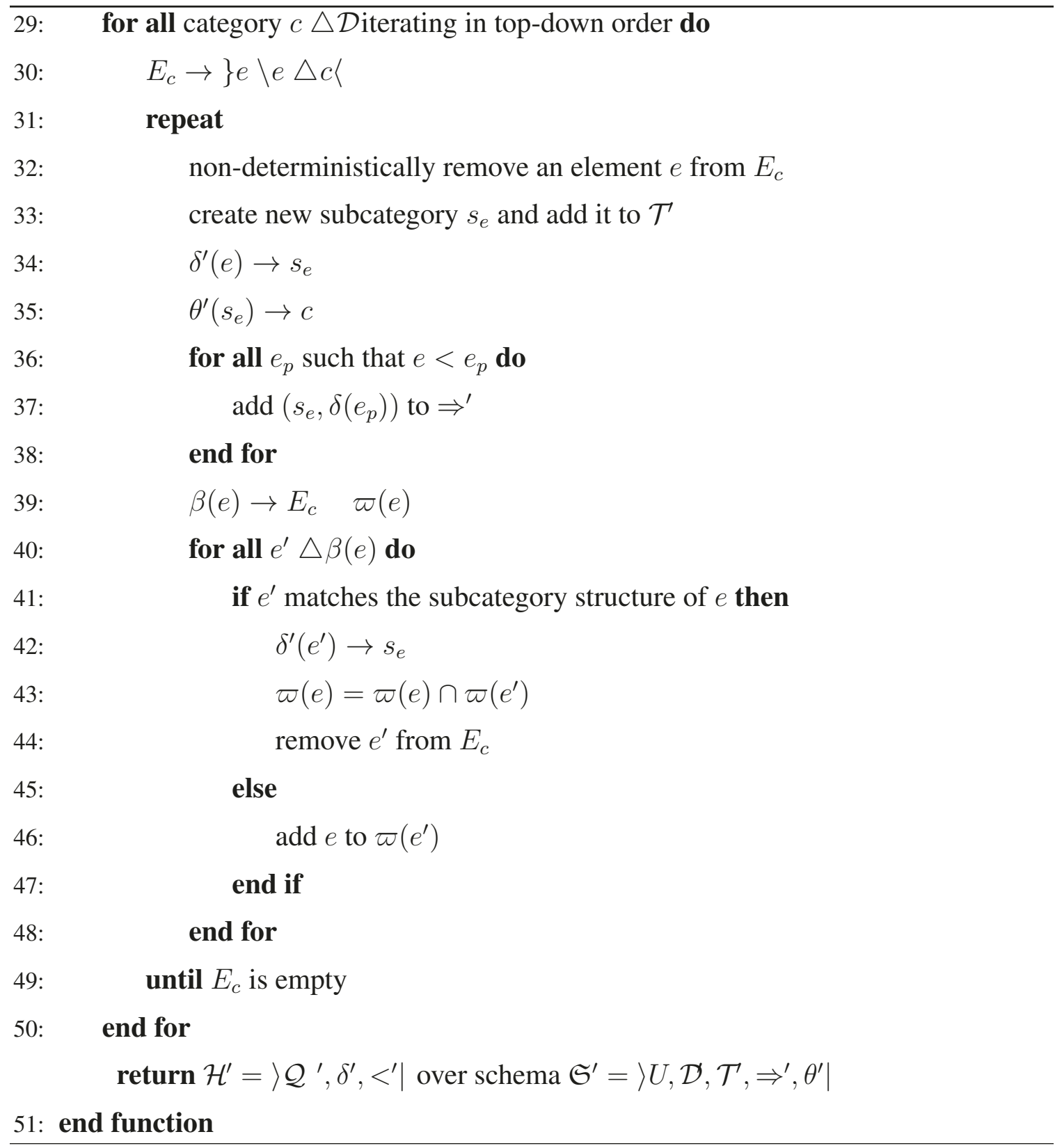


Proof: First, we have to check if $\mathcal{H}$ and $\mathcal{H}^{\prime}$ have the same elements, links and categories which can obviously be done in P-TIME. In addition, we need to check if $\Pi\left(\mathcal{H}, \mathcal{H}^{\prime}\right)$ is minimal. If $\Pi\left(\mathcal{H}, \mathcal{H}^{\prime}\right)$ is not minimal, then there have to be at least two subcategories that can be merged together causing $\Pi\left(\mathcal{H}, \mathcal{H}^{\prime}\right)$ to be a subset of its current value, without breaking the compliance condition. To check if this can happen, we can simply try all pairs of subcategories and check if they can be merged (without violating compliance). Two subcategories $s$ and $s^{\prime}$ can be merged if they roll up to the same parent subcategories (which can be checked in linear time wrt the schema size) and for all pairs of elements $e \triangle s$ and $e^{\prime} \triangle s^{\prime}$, we have $e \triangle \beta\left(e^{\prime}\right)$ (meaning that combining the two elements will not cause any non-functionality in subcategory roll ups). Using Algorithm 3, we can compute all consistency sets $\beta$ with P-TIME complexity in the size of the instance. Therefore, overall the decision algorithm takes P-TIME in the size of the dimension instance.

PCMs (and CMs) are compliant but not necessarily summarizable (although in many cases such as the PCM of Figure 1.4 they are summarizable as well). The following proposition provides a characterization of non-summarizable CMs by expressing a necessary (but not sufficient) condition for a CM result to be non-summarizable.

Proposition 14 In any non-summarizable compliant EHM instance there exists at least one pair of subcategories $s_{1}$ and $s_{2}$ such that:

1. the two subcategories have a shared parent (i.e. $s_{1} \Rightarrow p$ and $s_{2} \Rightarrow p$ for some subcategory $p$ ),

2. they have a shared descendant base subcategory (i.e. $b \Rightarrow s_{1}$ and $b \Rightarrow s_{2}$ for some base subcategory $b$ ), and

3. each has at least one descendant base subcategory that is not shared with the other (i.e. there exist base subcategories $b_{1}$ and $b_{2}$ such that $b_{1} \Rightarrow^{*} s_{1}$ and $b_{2} \Rightarrow^{*} s_{2}$ but neither $b_{1} \Rightarrow^{*} s_{2}$ nor $b_{2} \Rightarrow^{*} s_{1}$ ).

Proof: Lets consider a compliant EHM instance $\mathcal{H}$. We will show that either the equality condition of Proposition 6 holds for $\mathcal{H}$ implying that it is summarizable or the three conditions above altogether hold for at least one pair of subcategories. 
For every category $c$ of $\mathcal{H}$ first select all of the subcategories at the lower level as a potential set $\cup$ that satisfies the equality condition of Proposition 6. Obviously, for every subcategory $s$ of $c, b c v(s) \geq \sum_{s_{i} \in \mathcal{T}_{s}} b c v\left(s_{i}\right)$. Now, if there is a subcategory $s^{\prime}$ in $c$ for which the right hand side of the formula is strictly greater than the left hand side (i.e. $\left.b c v\left(s^{\prime}\right) \lesseqgtr \sum_{s_{i} \in \mathcal{T}_{s^{\prime}}} b c v\left(s_{i}\right)\right)$ this means that at least two subcategories $d_{1}, d_{2} \triangle \cup_{s^{\prime}}$ have a shared descendant base subcategory. Now, if $d_{1}$ and $d_{2}$ both have other descendant base subcategories as well, then the three conditions of the above Proposition hold for $d_{1}$ and $d_{2}$. Otherwise, we can remove the one that does not have any other descendant base subcategory from our initial selection of $\cup$ without violating the condition $b c v(s) \geq \sum_{s_{i} \in \mathcal{T}_{s}} b c v\left(s_{i}\right)$ for any subcategory $s$ of $c$ and handle other pairs like $d_{1}$ and $d_{2}$ in the same way, until no more pair remains.

Corollary 3 Any compliant EHM instance with less than three base subcategories is summarizable.

Proof: This is a direct result from Proposition 14. For the three conditions in the proposition to hold, the non-summarizable EHM instance must have at least three different base subcategories.

To enforce summarizability, we will apply an instance-based repair approach (Section 7.3) on results of preferred canonical mappings and show that this combined approach will produce more natural solutions with less computational cost (compared to the data repair approach).

\subsection{E-repairs}

In the canonical mapping process for an inconsistent HM instance, subcategories in the schema are adjusted so far to represent a correct model of the instance. In most cases, the resulting compliant instance is summarizable. There are exceptional cases where the result is still not summarizable (e.g. the canonical mapping of Figure 7.2). In the latter case, we propose to add/remove links between subcategories, and remove links between elements 

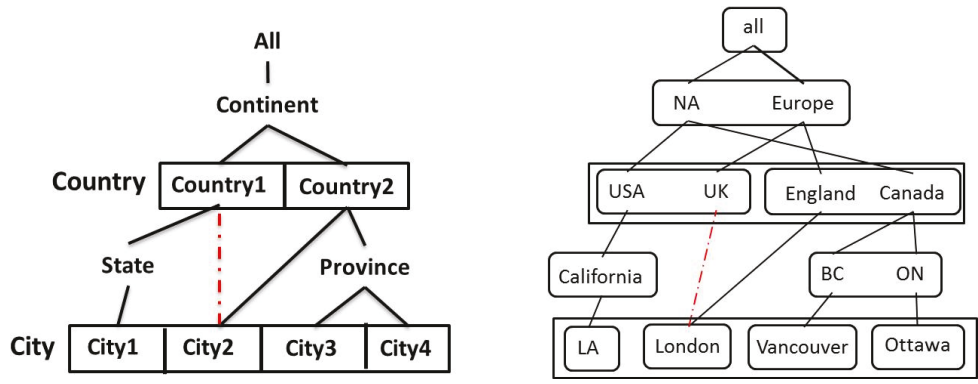

Figure 7.3: A minimal e-repair for the compliant EHM instance of Figure 7.2

of those subcategories accordingly, to enforce summarizability. The result is called an $e$ repair. As before, $\operatorname{dist}\left(\mathcal{H}, \mathcal{H}^{\prime}\right)$ denotes the symmetric difference between the sets of links in instances of dimensions $\mathcal{H}$ and $\mathcal{H}^{\prime}$, i.e. $\operatorname{dist}\left(\mathcal{H}, \mathcal{H}^{\prime}\right)=\left(\begin{array}{lll}<_{\mathcal{D}} & <_{\mathcal{D}^{\prime}}\end{array}\right) \cap\left(\begin{array}{ll}<_{\mathcal{D}^{\prime}} & <_{\mathcal{D}}\end{array}\right)$.

Definition 24 Given a compliant EHM instance $\mathcal{H}=\rangle \mathcal{Q}, \delta,<\mid$ over a schema $\mathfrak{S}=$ $\langle U, \mathcal{D}, \mathcal{T}, \Rightarrow, \theta|:$ (a) an e-repair of $\mathcal{H}$ is a compliant EHM instance $\left.\mathcal{H}^{\prime}=\right\rangle \mathcal{Q}, \delta,<^{\prime} \mid$ over schema $\left.\mathfrak{S}^{\prime}=\right\rangle U, \mathcal{D}, \mathcal{T}, \Rightarrow^{\prime}, \theta \mid$ such that:

1. $\mathcal{H}^{\prime}$ is summarizable (as characterized by Proposition 4), and

2. A new link $\left(s, s^{\prime}\right)$ in the subcategory schema of $\mathcal{H}^{\prime}$ can only be added if $\mathcal{H}$ contains $\left(s, s^{\prime \prime}\right)$ for some subcategory $s^{\prime \prime}$ with $\theta\left(s^{\prime}\right)=\theta\left(s^{\prime \prime}\right)$ (this means that in an e-repair, arbitrary assignment of links between subcategories from disconnected categories in the original dimension is prohibited).

(b) a minimal e-repair of $\mathcal{H}$ is an e-repair $\mathcal{H}^{\prime}$ such that $\backslash \operatorname{dist}\left(\mathcal{H}, \mathcal{H}^{\prime}\right) \backslash$ is minimal among all the e-repairs of $\mathcal{H}$.

Example 41 Figure 7.3 shows a minimal e-repair for the compliant (but non-summarizable) EHM instance of Figure 7.2 which is obtained by removing the link from London to UK in the dimension instance and the link between corresponding subcategories.

Proposition 15 There always exists at least one e-repair for any compliant EHM instance $\mathcal{H}$. If $\mathcal{H}$ is already summarizable, it will be the minimal e-repair of itself. 
Proof: We can always construct an e-repair $\mathcal{H}^{\prime}$ as follows: First, for each category $c$ we choose a specific element $e^{c} \triangle c$ (it does not matter which element) and let $<_{\mathcal{D}^{\prime}}=\mathcal{C}$, also let $\Rightarrow_{\mathcal{D}^{\prime}}=\mathcal{C}$. Then, for all categories $c_{i}$ and $c_{j}$ such that $c_{i} \Rightarrow_{\mathcal{D}} c_{j}$ and for every $a \triangle c_{i}$, we add $\left(a, e^{c_{j}}\right)$ to $<_{\mathcal{D}^{\prime}}$ and we add $\left(\delta(a), \delta\left(e^{c_{j}}\right)\right)$ to $\Rightarrow_{\mathcal{D}^{\prime}}$ (if not previously added). The dimension obtained is an e-repair since, by construction, satisfies the two condition of Proposition 4 for a category $c$ and any category $c^{\prime}$ that is a child of $c$ by choosing $\cup$ to be $\} \delta\left(e^{c^{\prime}}\right)\langle$. The first condition is satisfied because $\operatorname{dom}\left(\mathcal{S}_{b}^{c}(\mathcal{H})\right)$ contains all elements in base subcategory $b$ and $\operatorname{dom}\left(\mathcal{S}_{b}^{\mathcal{T}}(\mathcal{H})\right)$ also contains all those elements because they all roll up to both $e^{c}$ and $e^{c^{\prime}}$. The second condition is satisfied and relation $\mathcal{S}_{b}^{\mathcal{T}}(\mathcal{H})$ is a function because the second element of every tuple in that relation is $e^{c^{\prime}}$.

Example 42 The EHM instance of Figure 1.4 is already summarizable. Therefore, it is the only minimal e-repair of itself.

Proposition 16 There might exist exponential number of e-repairs for a compliant EHM instance.

Proof: An e-repair can be obtained by only adding/removing links between subcategories and links between corresponding elements (see Definition 24). To find all possible e-repairs we can simply try all possible combinations of links between subcategories. Assuming there are $k$ subcategories in the schema, each link may or may not be present between any child subcategory and a subcategory at the upper level. However, we can easily show that for any two subcategories $s_{c}$ and $s_{p}$, if $s_{c} \Rightarrow s_{p}$ then these subcategories can never have a shared parent (i.e. there is no $s_{a}$ such that $s_{c} \Rightarrow s_{a}$ and $s_{p} \Rightarrow s_{a}$ ). This means that the hierarchy of subcategories does not have any clique of size more than two. Therefore, according to Turán's theorem, the number of edges between subcategories in the case were all possible links are added (lets call this the fully connected schema) is $\frac{k^{2}}{4}$. Therefore, we can possibly $2^{\frac{k^{2}}{4}}$ potential e-repairs (choosing possible subsets of links from the fully connected schema).

When a query is posed to different e-repairs (regardless of whether it is computing results from scratch or through a pre-computed cube view at a lower level), the same query may 
produce different results based on the chosen e-repair. This is because e-repairs add/remove links from the dimension instance which in turn causes changes in the roll-up relations (and hence the query results). This opens the room for the notion of consistent query answers, i.e. answers that are shared between all minimal repairs [13]. If instead of choosing certain answers, we focus on a specific minimal e-repair, then query answers may vary depending on the specific repair that is chosen.

Starting from an inconsistent HM instance $\mathcal{H}$, we can obtain a summarizable EHM instance with the same categories and elements and with minimal link changes in the dimension instance through the following EHM-based repair process:

1. Find a preferred canonical mapping from $\mathcal{H}$ to $\mathcal{H}_{1}$.

2. Remove links between elements and subcategory of $\mathcal{H}_{1}$ to obtain a minimal e-repair $\mathcal{H}_{2}$.

The first step above may produce multiple choices. The FIND-PCM algorithm only produces one of the PCMs in polynomial time (producing all e-repairs results in higher complexity). Also, multiple minimal e-repairs can be obtained from the PCM chosen in the first step. However, as discussed later, all minimal e-repairs can be obtained only by processing the dimension schema (rather than the instance which is usually very large).

Consider the initial inconsistent HM instance of Figure 1.1, and the summarizable instances of Figures 1.4 and 7.3 which are results of the above process. A comparison between these results and the data repair of Figure 2.1 shows that through the data repair process we were forced to apply much more link changes. Hence, the e-repair process produces a more natural solution. This claim is formally expressed in the following proposition:

Proposition 17 Let $\mathcal{H}$ be an (inconsistent) $\mathrm{HM}$ instance and $\mathcal{H}_{1}$ be a minimal data repair of $\mathcal{H}$. There always exists an e-repair $\mathcal{H}_{2}$ for $\mathcal{H} \operatorname{such}$ that $\operatorname{dist}\left(\mathcal{H}, \mathcal{H}_{2}\right) \leq \operatorname{dist}\left(\mathcal{H}, \mathcal{H}_{1}\right)$.

Proof: Consider a dimension $\mathcal{H}_{C M}$ with same dimension instance as $\mathcal{H}$ but with one subcategory per element and with links connecting any two subcategories that have linked elements. $\mathcal{H}_{C M}$ is clearly a $\mathrm{CM}$ for $\mathcal{H}$ because all subcategory roll ups are relations with only one pair, and therefore they are total functions. 
Now, data repair $\mathcal{H}_{1}$ is obtained by removing set of links $L_{r}$ from instance $\mathcal{H}$ and adding set of links $L_{a}$ to it. We can create an e-repair as follows: remove all links $\left(e_{1}, e_{2}\right) \triangle L_{r}$ from $\mathcal{H}_{C M}$ and remove link $\left(\delta\left(e_{1}\right), \delta\left(e_{2}\right)\right)$ from the schema of $\mathcal{H}_{C M}$. Also, add all links in $\left(e_{1}, e_{2}\right) \triangle L_{a}$ to $\mathcal{H}_{C M}$ and add link $\left(\delta\left(e_{1}\right), \delta\left(e_{2}\right)\right)$ to the schema.

We can show that in the resulting dimension, let's call it $\mathcal{H}_{2}$, any category $c$ is summarizable from each $c^{\prime}$ that is a child of $c$. This is because, we know $c$ is summarizable from $c^{\prime}$ in $\mathcal{H}_{1}$. Clearly, $\mathcal{H}_{2}$ has the same elements and links in its instance as $\mathcal{H}_{1}$ (but with more subcategories). Therefore, any base element that rolls up to $c$ in $\mathcal{H}_{2}$, does that through one and only one element from $c^{\prime}$ (since we know this is the case for $\mathcal{H}_{1}$ ). Hence, $\mathcal{H}_{2}$ is summarizable.

Proposition 17 establishes a connection between data repairs and e-repairs. That is, for every data repair of an inconsistent HM instance, there exists an e-repair obtained by applying fewer (or equal) link changes compared to link changes of the data repair. Furthermore, we can apply the EHM-based repair process with less computational cost compared to the cost of finding a minimal data repair. This is expressed next.

Theorem 6 Finding all e-repairs for a compliant EHM instance takes $O\left(k^{2} \oplus 2^{\frac{k(k+4)}{4}}\right)$ time where $k$ is the size of the dimension schema (i.e. number of subcategories).

Proof: An e-repair can be obtained by only adding/removing links between subcategories and links between corresponding elements (see Definition 24). To find all possible e-repairs we can simply try all possible combinations of links between subcategories. Assuming there are $k$ subcategories in the schema, each link may or may not be present between any child subcategory and a subcategory at the upper level. However, we can easily show that for any two subcategories $s_{c}$ and $s_{p}$, if $s_{c} \Rightarrow s_{p}$ then these subcategories can never have a shared parent (i.e. there is no $s_{a}$ such that $s_{c} \Rightarrow s_{a}$ and $s_{p} \Rightarrow s_{a}$ ). This means that the hierarchy of subcategories does not have any clique of size more than two. Therefore, according to Turán's theorem, the number of edges between subcategories in the case were all possible links are added (lets call this the fully connected schema) is $\frac{k^{2}}{4}$. Therefore, we have to try $2^{\frac{k^{2}}{4}}$ potential e-repairs (choosing possible subsets of links from the fully connected schema). 
For each possible option, we have to check if it is actually an e-repair, i.e. whether it is summarizable. According to Corollary 1, this can be done in $O\left(k^{2} \oplus 2^{k}\right)$, leading to a combined complexity of $O\left(k^{2} \oplus 2^{k} \oplus 2^{\frac{k^{2}}{4}}\right)=O\left(k^{2} \oplus 2^{\frac{k(k+4)}{4}}\right)$. To check if an option is minimal, we only need to compute the total number of instance links that are associated to the subcategory links being removed. Notice that obtaining this number only requires meta data (in the form of a single integer) stored for each link in the schema (the total can be obtained by summation) and does not require processing the instance (However, if we were to generate rather than find the e-repairs, then an additional cost of iteration over instance links would be added). Hence, the overall computational complexity is still $O\left(k^{2} \oplus 2^{\frac{k(k+4)}{4}}\right)$.

\subsection{Discussion}

The purpose of the EHM-based repair process is to improve query answering performance. To summarize the implications of each step of the process for query answering, lets assume $\mathcal{H}_{H M}$ is an arbitrary (i.e. not necessarily summarizable) $\mathrm{HM}$ dimension, $\mathcal{H}_{E H M}$ is a compliant EHM dimension obtained through canonical mapping for $\mathcal{H}_{H M}$ and $\mathcal{H}_{\text {erep }}$ is a summarizable e-repair obtained for $\mathcal{H}_{E H M}$.

With an arbitrary $\mathrm{HM}$ dimension $\mathcal{H}_{H M}$, given an MD aggregate query $Q$, we can always compute correct answers to $Q$ directly from the fact table (even if $\mathcal{H}_{H M}$ is nonsummarizable). The problem is that the fact table is usually very large and such a computation from scratch will be impractical and takes considerable amount of time.

The alternative approach to answering $Q$ would be to derive the result from pre-computed cube views that are considerably smaller than the fact table. However, there is no guaranty that the derived result would be correct, i.e. equal to the result computed from scratch. In addition, there is no practical way to determine beforehand, whether the results would be equal (more precisely, the query answering problem defined in Section 6 is undecidable for arbitrary HM dimensions).

With a compliant EHM dimension $\mathcal{H}_{E H M}$ obtained through canonical mapping for $\mathcal{H}_{H M}$, as before, any query $Q$ can be computed from the fact table. Furthermore, $Q$ will yield the exact same results whether it is posed on $\mathcal{H}_{H M}$ or $\mathcal{H}_{E H M}$ because both dimensions 
have the exact same elements and links in their dimension instances. Also, there is no need for any syntactic modifications on $Q$ since $\mathcal{H}_{E H M}$ contains the exact same set of categories as $\mathcal{H}_{H M}$ in its schema. Moreover, the query answering problem is decidable for $\mathcal{H}_{E H M}$ and as long as the schema is of reasonable size, it will be practical to find a rewriting for $Q$ over a set of pre-computed cube views (assuming such a rewriting exists) and answer the query with better performance.

However, $\mathcal{H}_{E H M}$ may be non-summarizable in exceptional cases. So, even if we have a complete set of pre-computed cube views over children of categories in $Q$, there is the possibility that no rewriting exists for $Q$, in which case we would have compute the query result from scratch.

With a summarizable dimension $\mathcal{H}_{\text {erep }}$, obtained through the EHM repair process, not only the query answering problem is decidable, but also given a complete set of precomputed cube views, it is guarantied that a rewriting will exist for $Q$. A drawback of $\mathcal{H}_{\text {erep }}$ is that if we pose the same query $Q$ to both $\mathcal{H}_{H M}$ and $\mathcal{H}_{\text {erep }}$, we may not obtain the exact same results (unlike $\mathcal{H}_{E H M}$ that would produce the same results). This is because, the instance of $\mathcal{H}_{\text {erep }}$ has been slightly modified by adding/removing links in its dimension instance (this same drawback will be magnified with data repairs, since they cause much more changes in the dimension instance). 


\section{Chapter 8}

\section{Experiments}

\subsection{Introduction}

Like most software, OLAP tools are validated through testing. However, unlike many other software tools, testing OLAP systems needs large data sets with special characteristics. For example, evaluating the performance of multidimensional models requires a variety of large data sets with (summarizable or non-summarizable) dimensions that have different graph structures and statistical properties. At the same time, due to privacy and proprietary concerns, structurally rich data sets from real industrial applications are hard to come by [64].

Efforts have been made to develop powerful and expressive data set generators [6, 14, $16,34,36,58,67]$. However, most of these synthesizers can only generate simple treeshaped dimensions, but not more complicated DAG structures (as in the dimension model of HM).

In [70], a model-based approach to synthesizing structures based on DAG for systematic testing and evaluation of decision support software has been proposed. The authors present a framework that provides a small, expressive language for specifying such models. Unfortunately, the language only supports categories as first-class elements of a schema. As a result, it is not capable of declaring subcategories required for expressing HM models.

We have developed a Java-based framework for our experimental evaluations on HM and EHM models. The framework allows us to create dimensions with complicated structures and instances that may contain different types of inconsistencies. The framework also enables us to run our creation and repair algorithms and evaluate the cost of generating EHM instances versus the benefits they provide for improving query answering performance. 


\subsection{Experimental Setting}

All of our experiments are executed on a system with Intel Core 2 Duo 2.10GHz processor and 4GB of RAM. The underlying implementation of the multidimensional databases are ROLAP schemas created and instantiated using MySQL Workbench version 6.1.6 and queried through our Java-based framework using JDBC.

To declare HM models, our framework takes as input a description of categories and links in the schema. This input is provided in the form of simple Java entities or optionally as XML input and contains for each category, its name plus the parent categories it is connected to.

Furthermore, for the description of an HM instance, our framework receives the shape (i.e. a set of statistical constraints on the distribution properties) and the size of the desired instance. Based on the specified description, the framework creates ROLAP schemas in the form of star tables ${ }^{1}$ for the declared HM dimensions and populates them with records that correspond to the specification of the dimension instance.

We have included in our framework the implementation of the FIND-PCM algorithm (see Section 7.2.1). Provided the description of an HM dimension, the framework automatically finds a preferred canonical mapping (and creates the corresponding compliant EHM dimension). This includes creating ROLAP schemas for newly introduced subcategories as well as the population of subcategory tables with records that indicate membership of elements to their subcategories (as in Section 4.3).

Our experiments evaluate the cost of creating HM dimensions versus converting them to compliant EHM dimensions. In addition, we will compare the resulting (HM and EHM) models in terms of query answering performance using different types of queries. There are a set of factors that can act as constant or variable settings of each experiment including:

$\subseteq$ The number of instances: Dimension instances (and dimension tables in a ROLAP implementation) are usually much smaller than fact tables in terms of the number of records. But they can still vary greatly in size and range from small instances with tens or hundreds of records, to very large instances with hundreds of thousands of

\footnotetext{
${ }^{1}$ In our implementation of star, each base element corresponds to one row of the star table, and if it has multiple parents in some upper level category, this is handled by a simulation of multi-valued cells using delimiters
} 
elements. Obviously, the dimension instance size is the most important factor in the time complexity of creation and repair algorithms as well as query answering.

$\subseteq$ The distribution of elements within categories: Another factor that can impact the experiment results is the relative distribution of elements within different levels (or categories) of the schema. In the process of populating an instance, our framework allows us to determine this factor by assigning to each category a distribution measure relative to the base category. A distribution measure of $m$ for category $c$ indicates that on average every $m$ base elements roll up to one element from category $c$.

$\subseteq$ The number of inconsistencies (and inconsistency types): The number of base elements that cause some sort of inconsistency (in the form of heterogeneity or nonstrictness) through their upward path in the dimension instance can potentially impact the costs of creating and specially repairing HM dimensions. We can define new inconsistencies in our framework by specifying the type of inconsistency (nonstrictness or heterogeneity), the categories at which the inconsistency occurs and the number (or rate) of base elements that encounter the inconsistency through their upward path.

\subsection{Creating HM Dimensions vs Finding Preferred Canonical Mappings}

Our first set of experiments demonstrate the effect of dimension instance size on the time it takes to create HM instances and the time it takes to transform them into compliant EHM instances using the FIND-PCM algorithm. We have chosen the running example of the Location HM dimension from Figure 1.1a for this purpose and tried multiple dimension instance sizes.

For the first set of experiments we will keep the distribution and inconsistency rates (see Section 8.2) constant and modify the instance size to see the effects. More specifically, we have experimented with the creation of small to large instances ranging from 200 elements to 200000 elements per instance. The rate of inconsistency for all the different instances of this set of experiments is fixed at $5 \%$ including both non-strictness and heterogeneity. In other words, at random, for every 100 base elements (cities) the data generator creates on average 5 elements that cause either non-strictness or heterogeneity in their upward path 


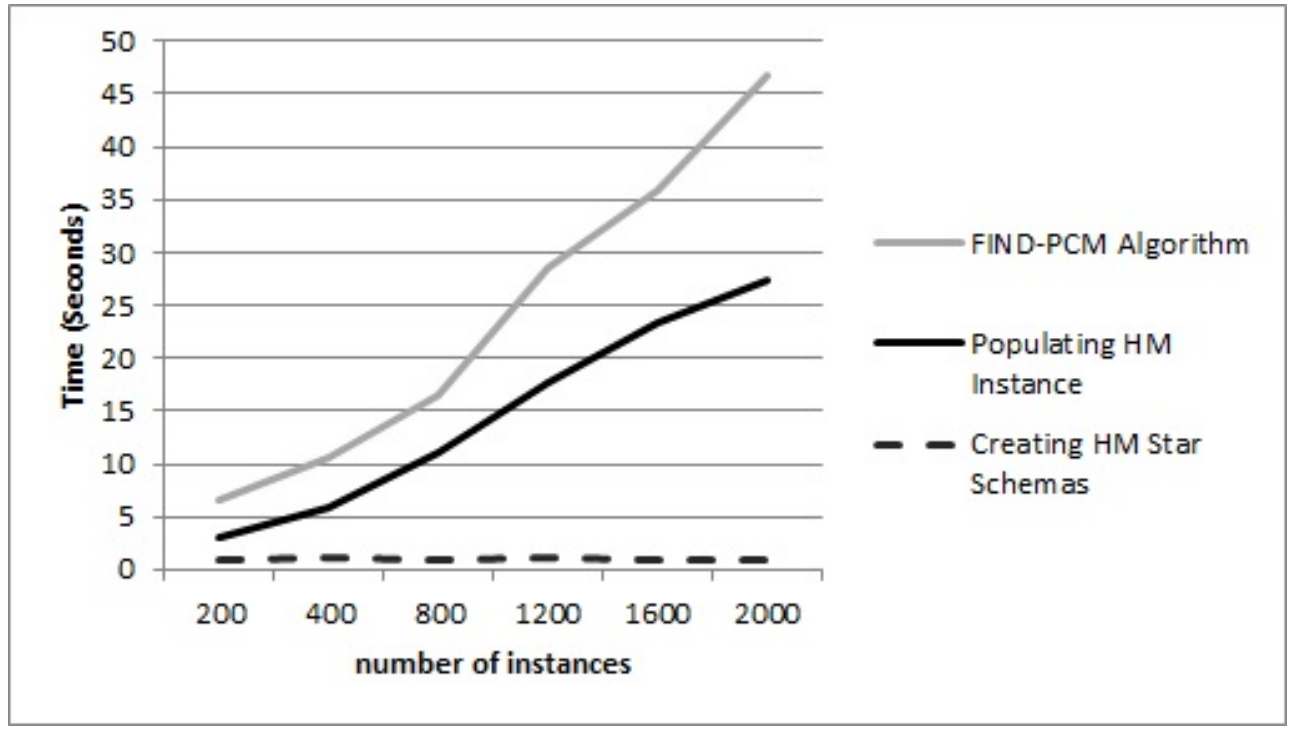

Figure 8.1: Instance size vs dimension creation time (for small instances)

through the dimension instance. In addition, the distribution of elements within categories is fixed: for every 150 cities, we have populated the instance with 30 provinces, 50 states, 5 countries and 1 continent (as expected, regardless of instance size, there is only one element assigned to category $\mathrm{All}$ ).

The results are shown in Figures 8.1 (for smaller instances) and 8.2 (for larger instances). The horizontal axis captures the number of instances and the vertical axis shows time in seconds. The dashed graph represents the time it takes to create star schema for the HM dimension schema of Location. Our framework automatically generates the following query from the input description of the dimension schema to create the Location star table:

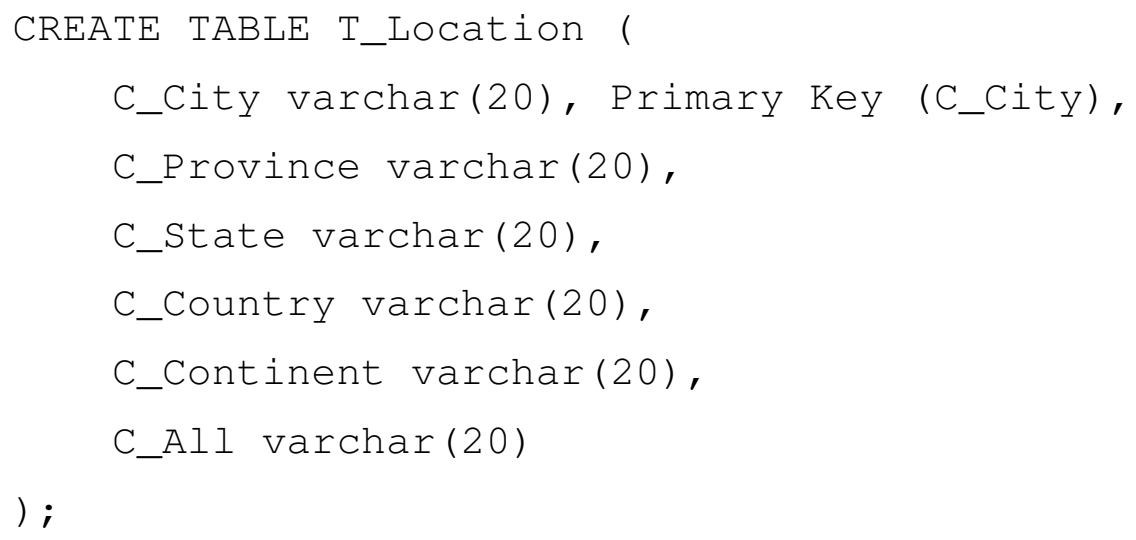




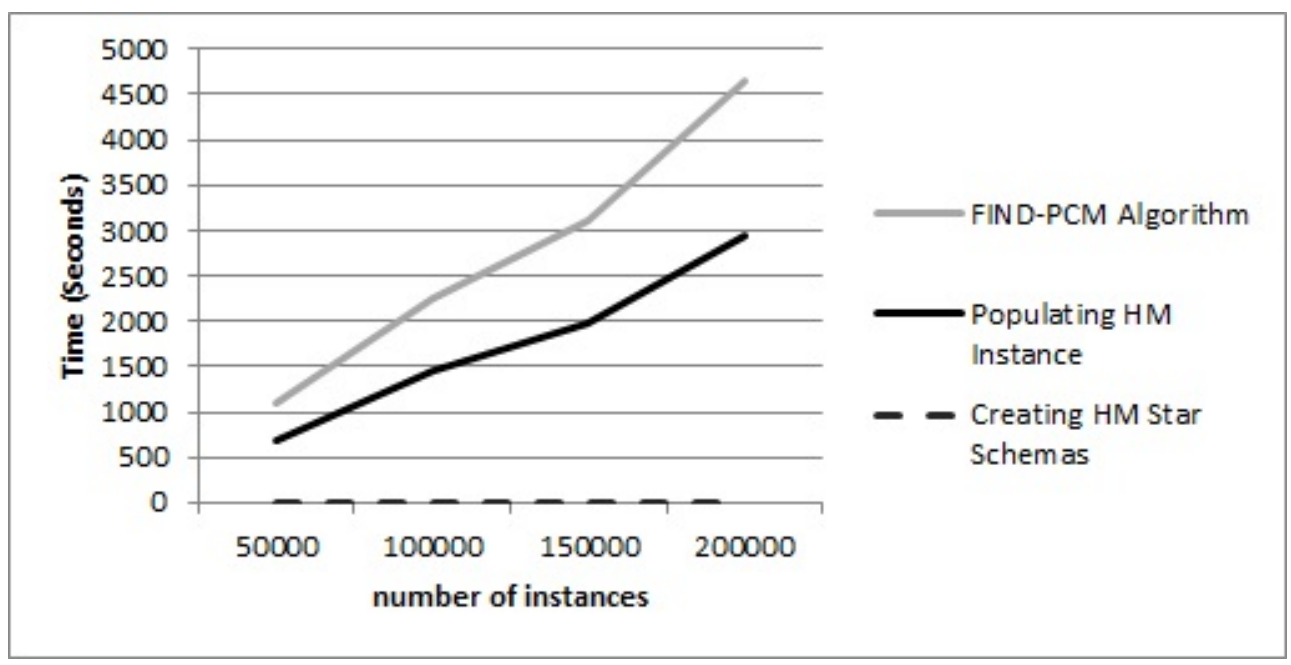

Figure 8.2: Instance size vs dimension creation time (for large instances)

The solid graph in black shows the time it takes to create (populate) HM instances and the solid gray graph depicts the time to transform the resulting instance into a compliant EHM instance using the FIND-PCM algorithm. A simple analysis of these graphs shows that the cost in terms of time of both creating HM instances and transforming them into compliant EHM instances (using FIND-PCM) linearly grows as the number of instances increases. This is regardless of whether the instance is small or very large. Also, our implementation of the FIND-PCM algorithm for creating compliant EHM instances takes about twice as much time as the time required for creating an HM instance.

Our next set of experiments demonstrate the effect of the schema size (number of categories) on the creation of HM instances and on running the FIND-PCM algorithm. More specifically, we will vary the number of categories in the Location HM schema from 3 categories (City, Country and All) to 9, by adding categories one by one to lower levels of the schema (Figure 8.3a) and then starting over and adding categories at higher levels of the schema (Figure 8.3b). As expected, if new categories are added to lower levels (e.g. adding a Branch category below City), the effect on the running time of FIND-PCM will be significantly higher compared to the case where categories are added at higher levels (e.g. adding a new category above Country). This is because an inconsistency at the level of a category $c$ can potentially cause the introduction of new subcategories at the level of categories below $c$ but not at the level of higher categories. In this set of experiments, the instance size is fixed at 10000 elements, and as before the rate of inconsistency is fixed at 
$5 \%$ including both non-strictness and heterogeneity.

\subsection{Query Answering Performance}

Our next set of experiments demonstrate how the addition of subcategories in the EHM model can improve query answering performance by enabling the use of certain pre-computed cube views that are not re-usable in an HM setting. We have chosen an MD database composed of four HM dimensions, namely Location, Date, Product and Employee, as depicted by Figure 8.4 .

Now, a star implementation of our HM dimensions will be composed of the following relational schemas (for simplicity and without loss of generality, we are assuming that the fact table contains only one measure, namely sales):

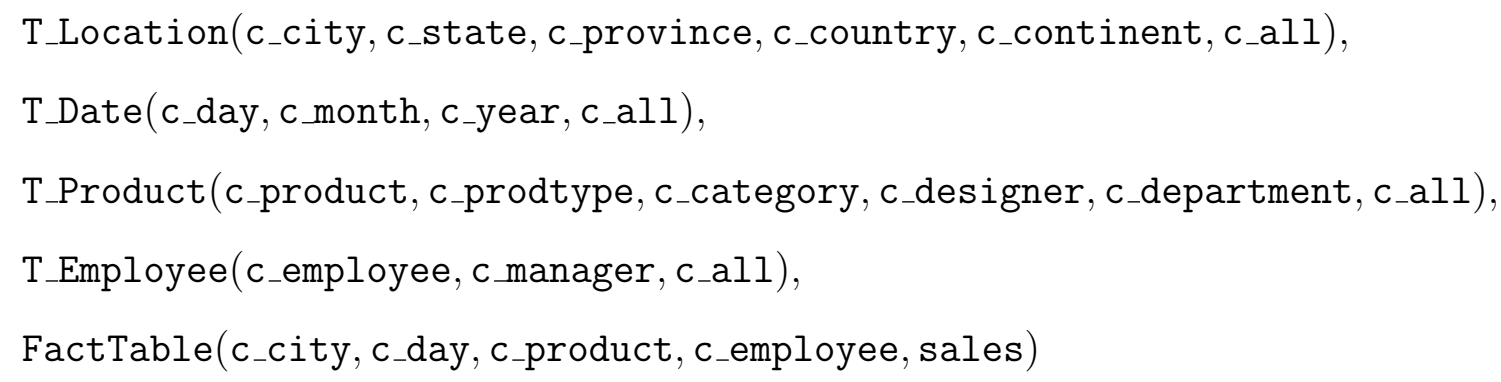

Using our data generating framework, we have loaded each dimension with sizeable amount of test data. Specifically, for this set of experiments, we have populated the Location dimension with 200000 elements, the Date dimension with 4000 elements, the Product dimension with 100000 elements, and the Employee dimension with 20000 elements. In addition, we have instantiated the fact table with one million facts.

As before, our implementation of the FIND-PCM algorithm is used to transform the inconsistent HM dimension of Location into a compliant EHM instance which results in the creation of the following subcategory tables ${ }^{2}$ :

$$
\begin{aligned}
& \text { Country_Subcategories(c_element, country1, country2), } \\
& \text { City_Subcategories(c_element, city1, city2, city3) }
\end{aligned}
$$

\footnotetext{
${ }^{2}$ In reality some employees may have multiple managers and some products may be created by multiple designers (or even none). Therefore, these dimensions may be subject to inconsistencies and require transformation to compliant EHM instances. However, for simplicity of queries, we will assume that Date, Product and Employee are already consistent dimensions.
} 


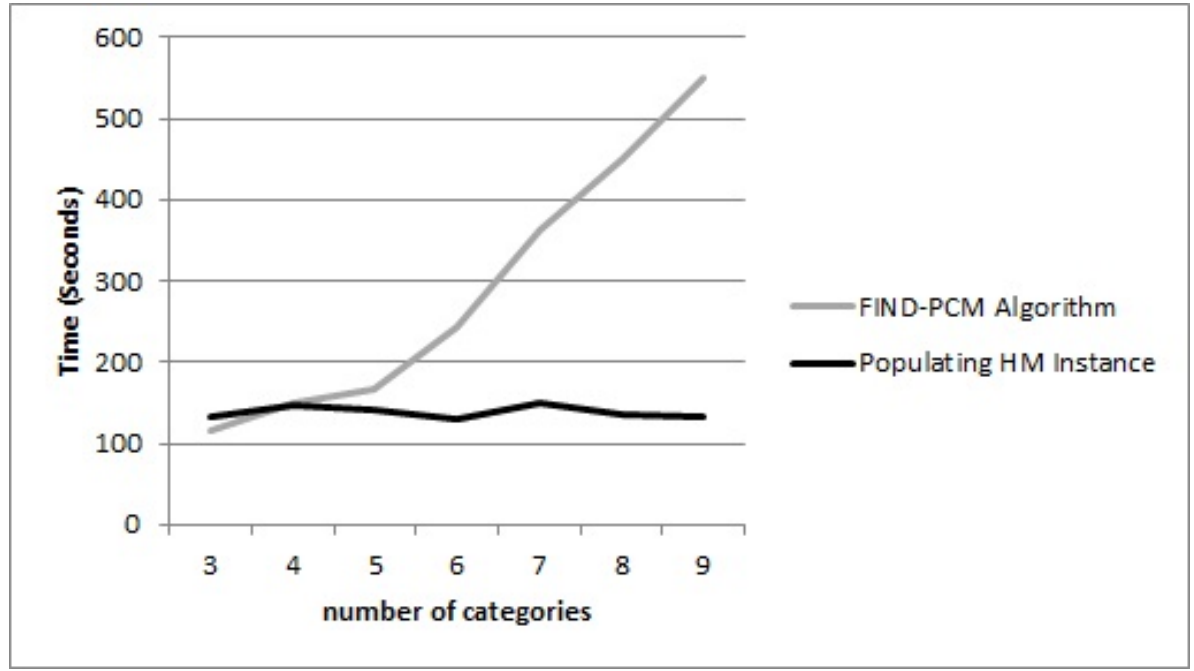

(a) Adding categories at lower levels

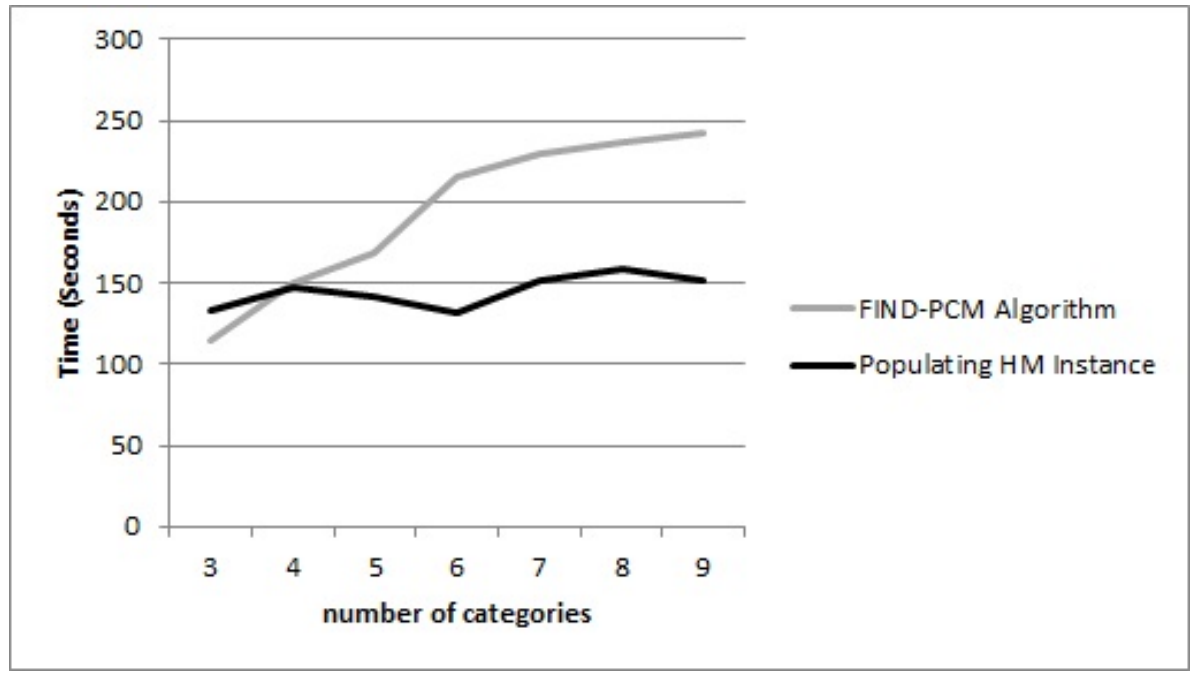

(b) Adding categories at higher levels

Figure 8.3: The effect of adding categories on creation time 

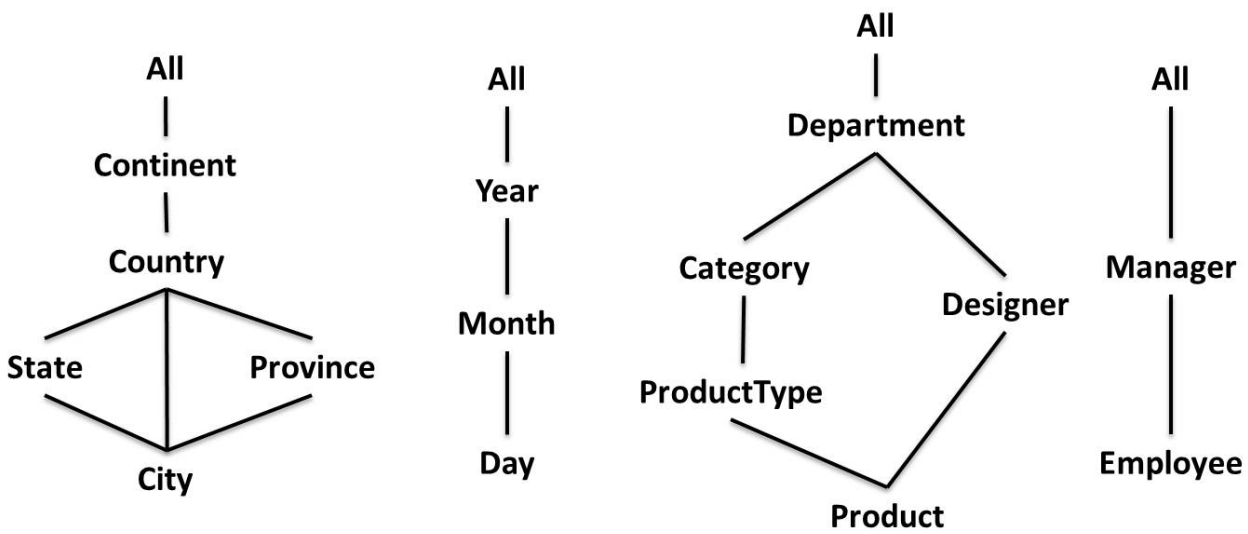

Figure 8.4: HM schemas for dimensions Location, Date, Product and Employee

Our next experiment is conducted as follows: We have materialized View_cou_mon_emp_pty = $\Psi_{S U M}[$ Country, Month, ProdType, Employee], a pre-computed cube view containing monthly sales made by each employee for every product type in each country. Having this materialized view, we have compared the time required for answering the following set of queries in HM and EHM (notice that in HM, because of the inconsistency of Location, category Continent is not summarizable from category Country, therefore any query involving Continent must be computed from scratch):

1. $\Psi_{S U M}[$ Continent] is a one-dimensional query that computes total sales values for different continents. In HM, this query is expressed as:

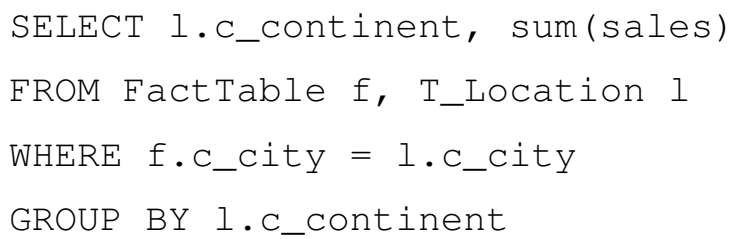

In the EHM setting, we can reuse the materalized view as follows:

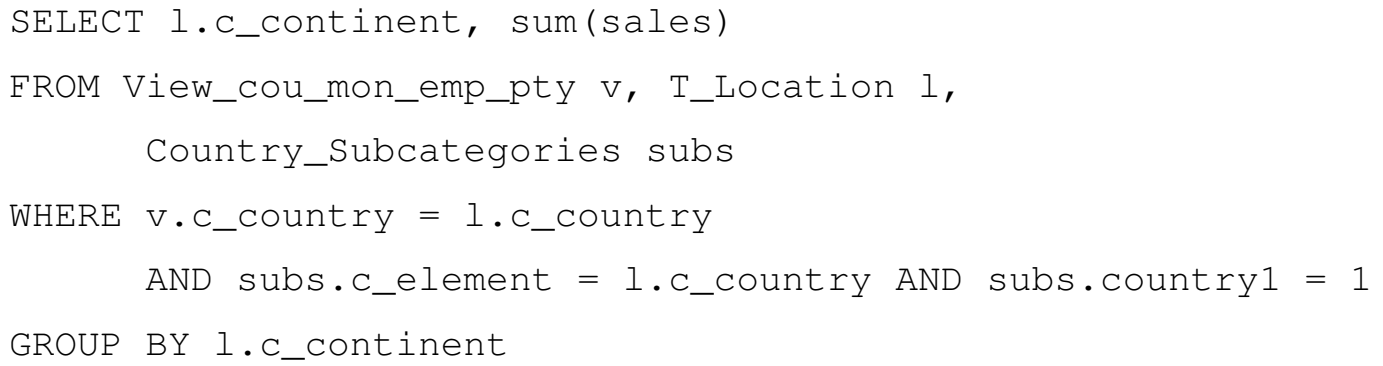


notice the addition of the subcategory table to filter elements of subcategory Country 1

2. $\Psi_{S U M}[$ Continent, Year] is a two-dimensional query that computes annual sales for different continents. In HM, this query is expressed as:

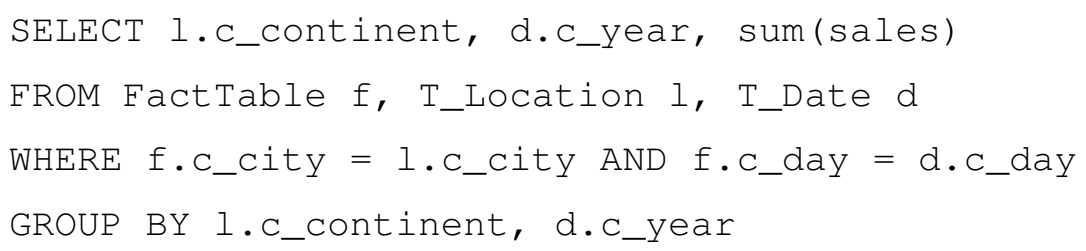

In the EHM setting, the query is expressed as follows:

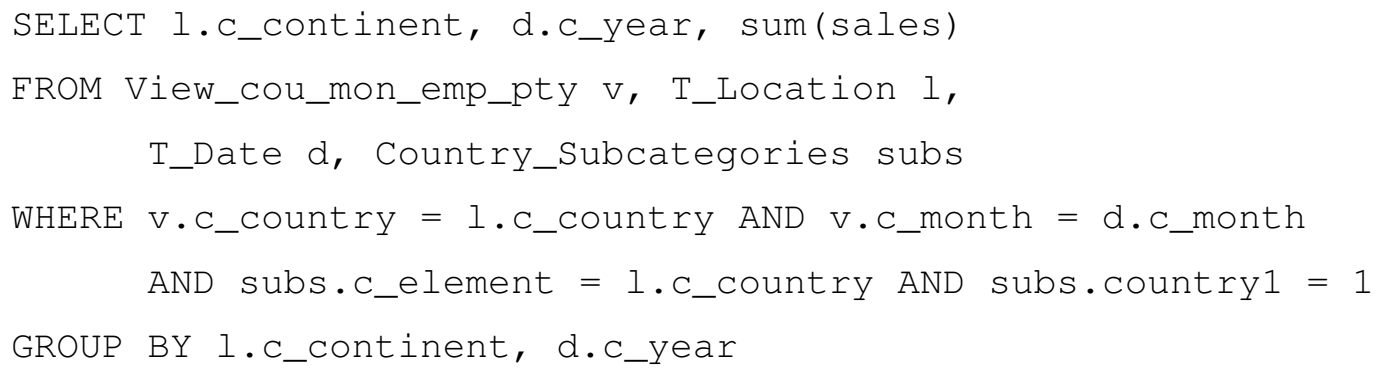

3. $\Psi_{S U M}[$ Continent, Year, ProductType] is a three-dimensional query that computes annual sales values for different continents by product type. In HM, this query is expressed as:

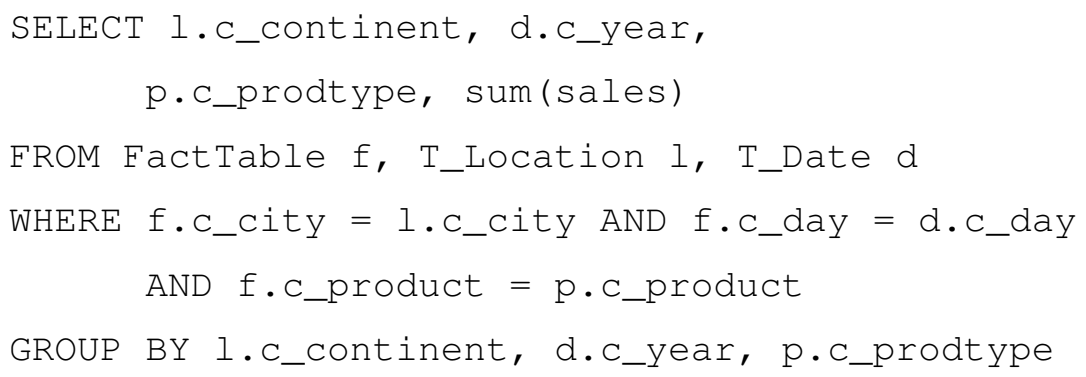

In the EHM setting, we can reuse the materalized view as follows:

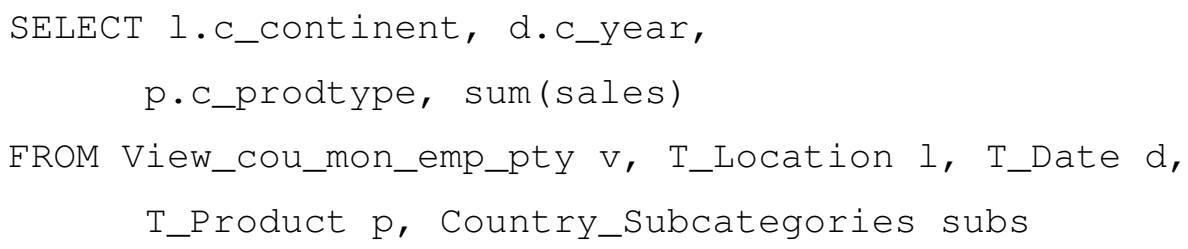


WHERE V.C_country $=$ l.c_country AND V.c_month $=$ d.c_month AND V.c_prodtype $=$ p.c_prodtype AND subs.c_element $=1$.c_country AND subs.countryl $=1$ GROUP BY 1.c_continent, d.c_year, p.c_prodtype

4. $\Psi_{S U M}[$ Continent, Year, Employee] is another three-dimensional query that computes annual sales values for different continents by each employee. In HM, this query is expressed as:

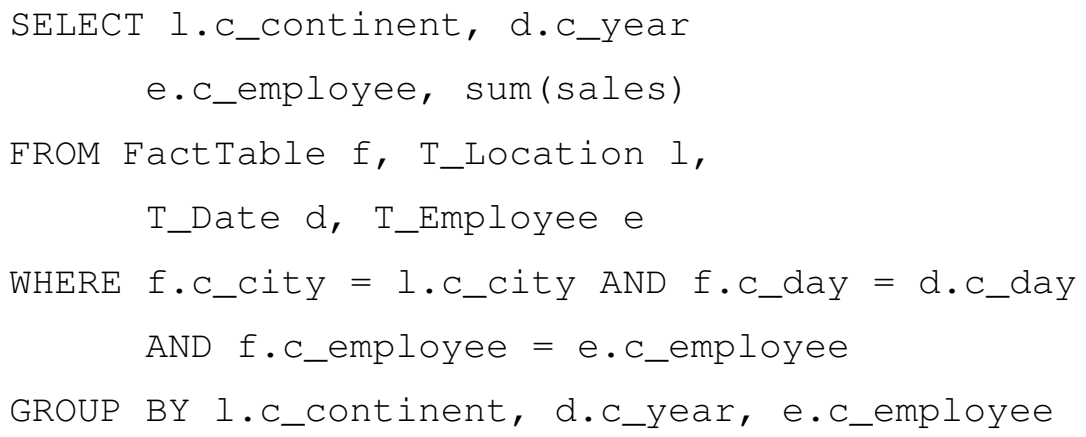

In the EHM setting, the query is expressed as follows:

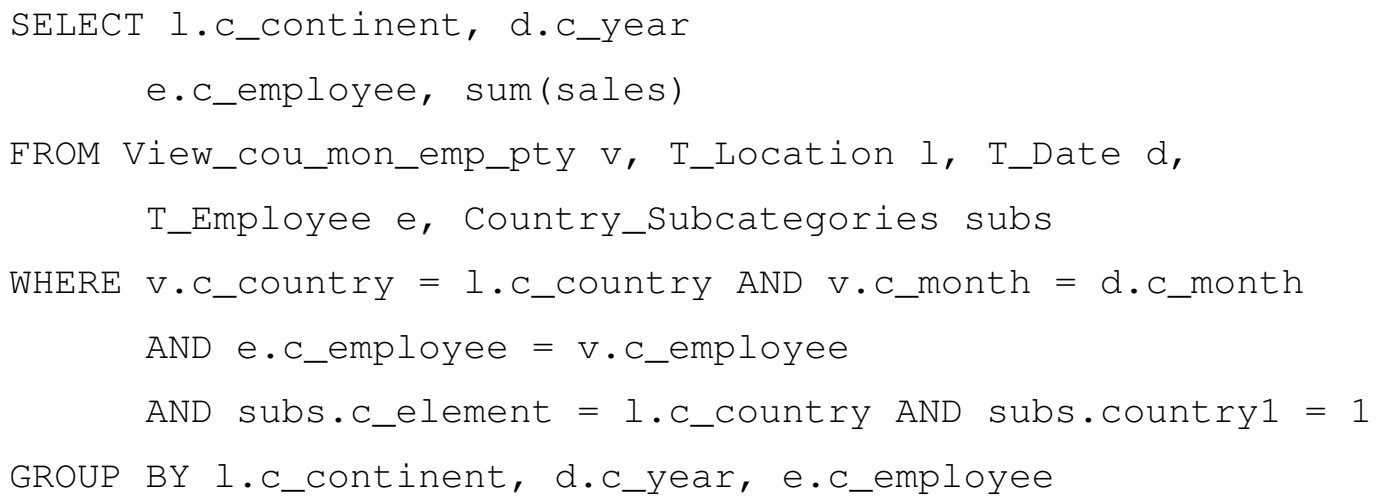

5. Similarly, $\Psi_{S U M}[$ Year, ProductType, Employee $]$ is expressed as:

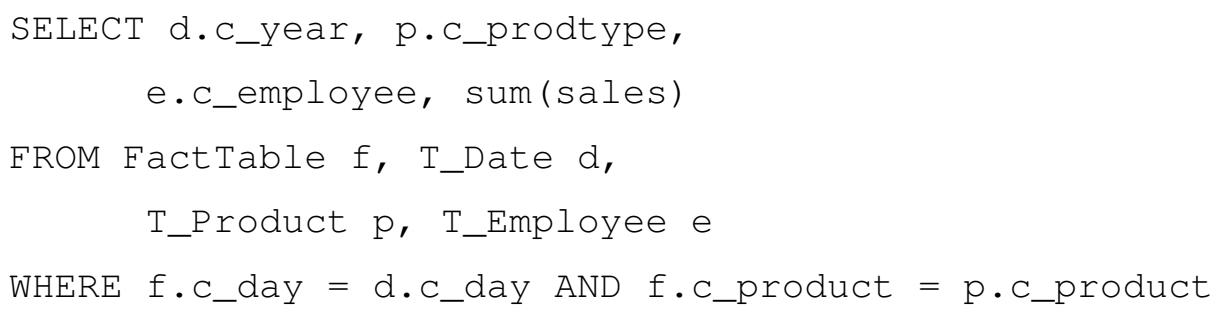




\author{
AND f.c_employee = e.c_employee \\ GROUP BY d.c_year, p.c_prodtype, e.c_employee
}

And in the EHM setting it is expressed as follows:

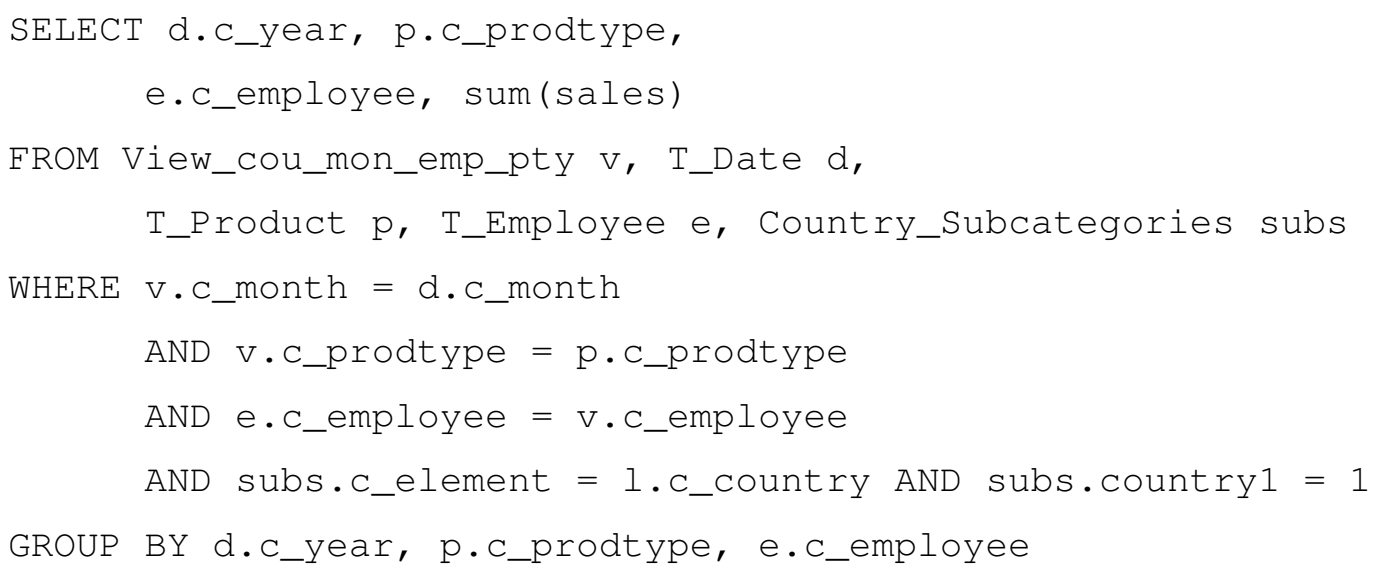

6. Finally, $\Psi_{S U M}[$ Continent, Year, ProductType, Employee] computes annual sales values made by each employee for different product types in different continents. In $\mathrm{HM}$, this query is expressed as:

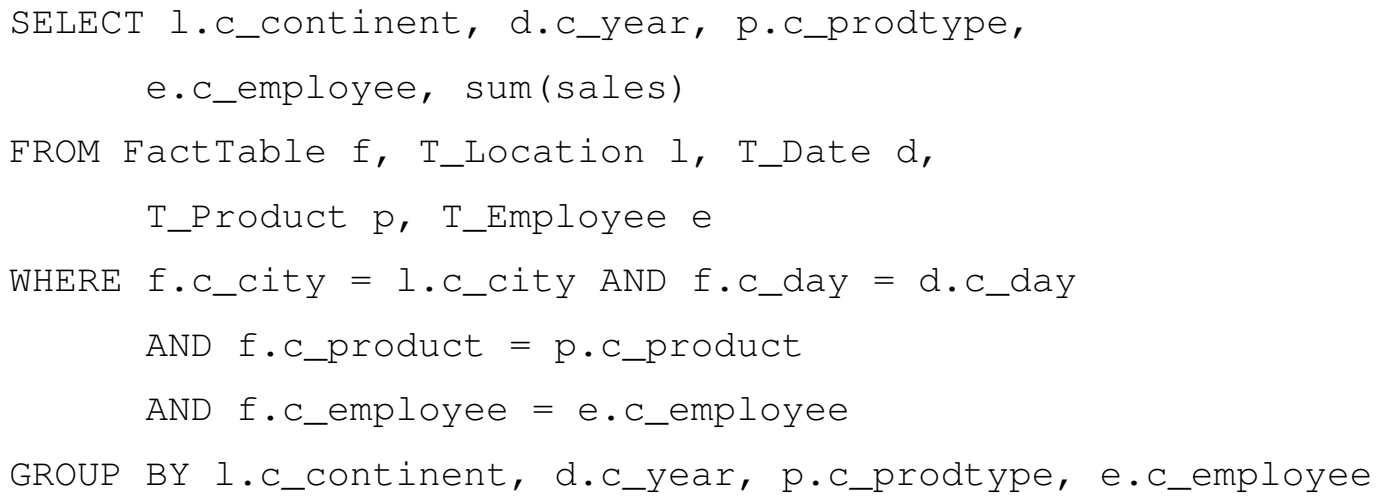

In the EHM setting, we can reuse the materalized view as follows:

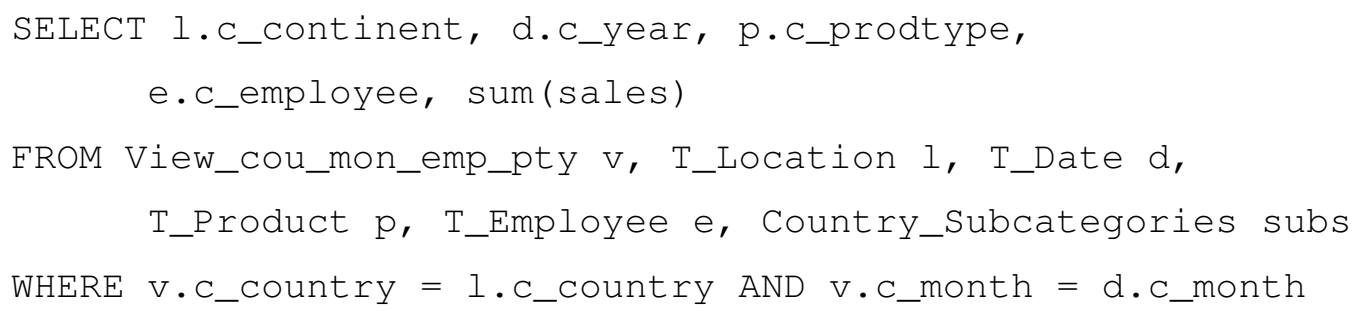




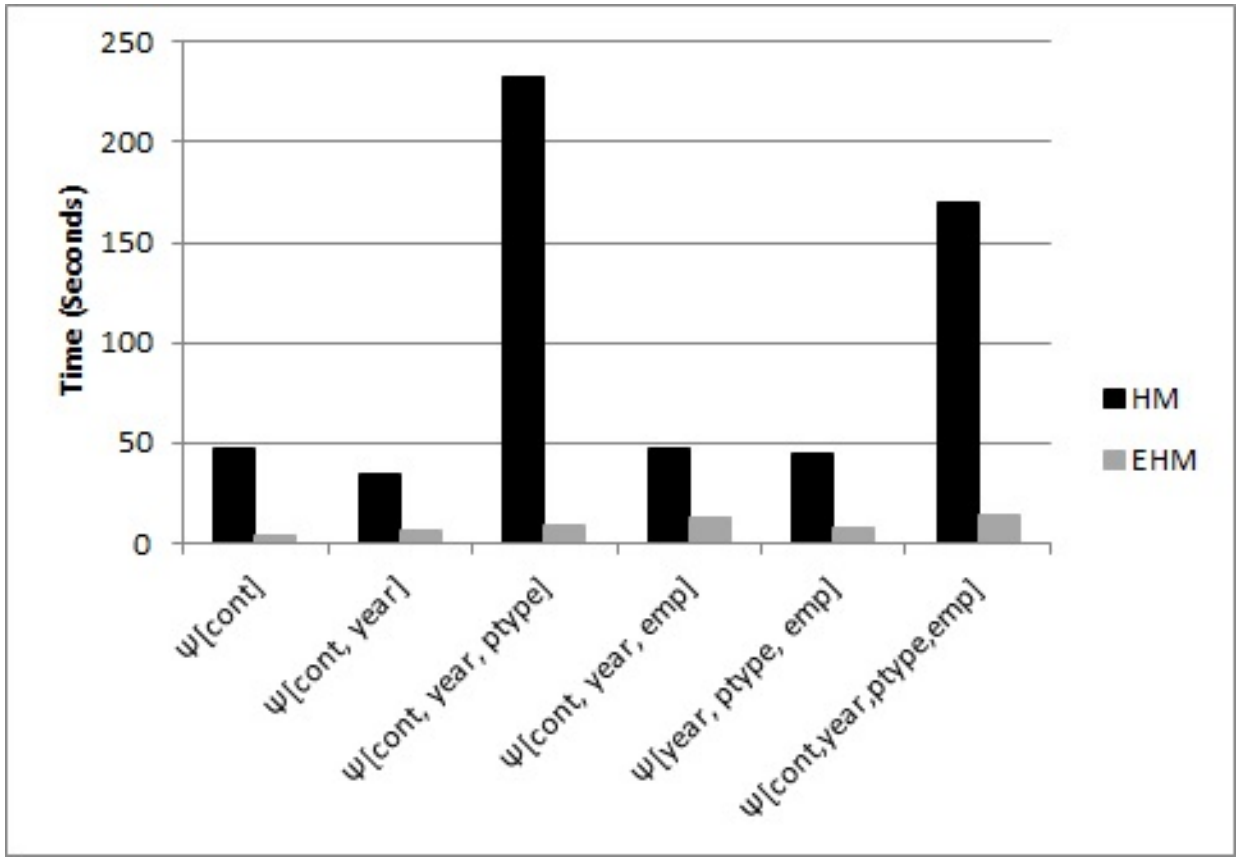

Figure 8.5: Query answering time for selected queries

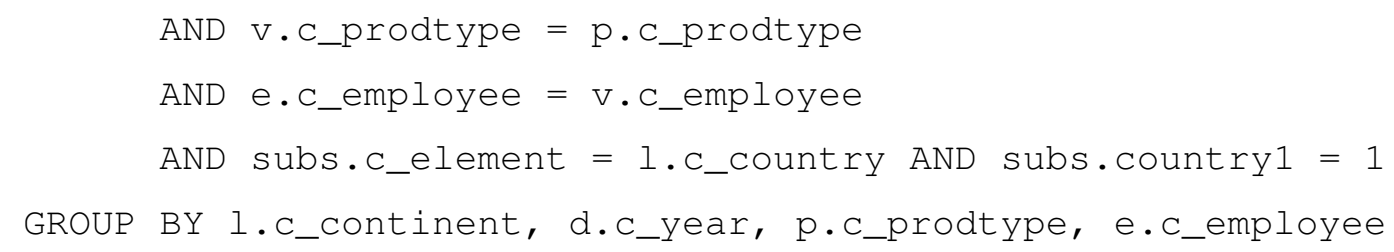

Figure 8.5 shows the results of running the above queries in the aforementioned multidimensional setting. The immediate result of is that, as expected, the improvement in query answering performance provided by the addition of subcategories in EHM (and consequently the ability to reuse additional pre-computed cube views) is significant. This is specially the case when dealing with relatively large fact tables.

A closer look at the results of Figure 8.5 also shows that the inclusion of larger dimension such as Location and Product generally has a bigger impact on query answering compared to smaller dimensions such as Time and Employee.

Our final set of experiments demonstrate the impact of fact table size on query answering time for our selected queries over HM and EHM. Specifically, we have computed the result of $\Psi_{S U M}[$ Continent, Year, Employee] over different fact table sizes ranging from 20000 to 1000000 records. The result is shown in Figure 8.6. Notice that, in the 


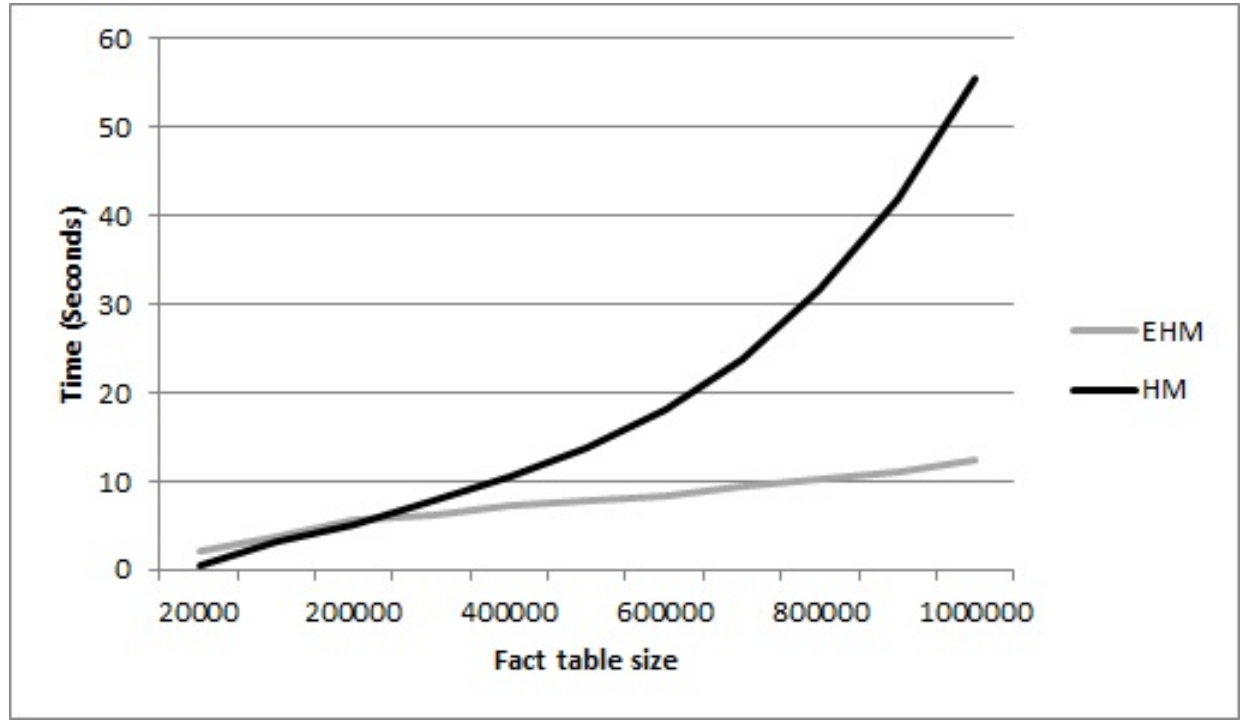

Figure 8.6: The impact of fact table size on query answering time

case of EHM dimensions, results have been computed through materialized cube view View_cou_mon_emp_pty and that the view size grows in slower rate compared to the growth rate of the fact table. Our results clearly show that with larger fact tables, the improvement in query answering time provided by the use of EHM models is much more significant. 


\section{Chapter 9}

\section{Conclusions and Future Work}

\subsection{Conclusions}

In this thesis, we have focused on creating MD models subject to summarizability. Previous research has mainly focused on restoring summarizability through changes made to elements of dimension instances or the relationships/links between those elements. Inspired by the shortcomings of previous approaches, we proposed a new MD model, as an extension of the HM model, that is more expressive in capturing dimensions subject to summarizability. Subcategories are the main ingredient of this extension. With the introduction of subcategories, we can capture the exact structure of the dimension instance in a much higher granularity than the granularity of the dimension instance which reduces size of the structure. This in turn enables us to enforce summarizability conditions without having to modify user-defined categories and with limited or no changes in the dimension instance.

We formalized a new notion of summarizability that applies to both HM and EHM dimensions. Summarizable HM dimensions are still summarizable in EHM without change. However, the inverse is not true. There exist real-world domains for which summarizable EHM dimensions exist, however they cannot be modelled in a summarizable way using HM.

The ADD-LINK algorithm provided in Section 4.2.1 produces EHM dimensions from scratch by gradually adding links including endpoints. In the exceptional case where the new link does not obey the structure of the subcategory hierarchy, new subcategories are added accordingly.

We have provided a formal characterization of expressiveness for classes of dimension models. We have established that the class of summarizable HM dimensions is less expressive than that of summarizable EHM dimensions. There are many commonly used 
dimensions for which the HM metamodel fails to accommodate an MD model, including instances, that satisfies summarizability. At the same time they can be modelled in a summarizable by adding subcategories in the schema.

One of the important issues in improving query answering performance for MD databases is to choose a set of cube views for materialization when it is too expensive to materialize all cube views [33]. Later, when another aggregate query needs to be computed, we need to be able to decide whether this new query can be computed using the set of materialized pre-computed cube views. To make this latter decision, we need an efficient algorithm that given a cube view can determine from which pre-computed cube views it can be computed. With the HM model such an algorithm will have to process the dimension instance which is usually very large and makes the computation impractical. With the help of the constraints that exist in the EHM model, we have designed such an algorithm for compliant EHM dimensions that runs in constant time with regard to the instance size.

We formally characterized the problem of query answering using views in an MD setting. We express MD aggregate queries and their rewritings using the formalism of Datalog with aggregation. We have shown that given a set of materialized pre-computed cube views in an EHM setting, we can effectively rewrite new queries in terms of available views and obtain query answers with better performance.

We proposed a repair approach based on the EHM model that starting from a nonsummarizable HM dimension, introduces subcategories into the schema in to produce a compliant EHM dimension without any modification to the dimension instance or categories in the schema. Then, in exceptional case where the result is not summarizable, links in the dimension instance are modified to enforce summarizability. In comparison with the existing data repair approaches, our approach incurs in lower computational cost. In addition, we have shown that the result of the EHM-based repair process is more natural compared to existing repair approaches.

As demonstrated by our experiments, the EHM model performs well when the schema size is relatively small. Specially in the case of computing summarizability sets and query answering using pre-computed cube views, since the execution time of our algorithms grow exponentially with schema size, having a very large number of categories and subcategories will make these algorithms impractical. In these cases we may need to take advantage of 
approximation algorithms or partial computation of outputs.

\subsection{Future Work}

We conclude this thesis with a list of problems for further research:

$\subseteq$ Our COMPUTE-SUMSETS algorithm from Section 5.3 can be modified to compute summarizability sets for a subset of categories as opposed to all of them. This will result in lower complexity since the execution time of this algorithm grows exponentially with the size of subcategories. A (manual or automated) analysis of the history of queries imposed on the schema can direct the partial evaluation (to determine which categories reused more often and select them for the computation of summarizability sets).

$\subseteq$ An alternative to computing summarizability sets from scratch would be to compute them once and then check after any update, to see if the sets require modification. Specially, in the case of HM dimensions where computing summarizability sets before queries is not efficient, this might be a viable solution that requires further investigation.

$\subseteq$ It would be interesting to research the possible use of ontologies for designing EHM dimensions. Semantic constraints on the subcategory category (as well as the category hierarchy) can be checked using ontological constraints. The use of ontologies can also aid the automation of the EHM repair process.

$\subseteq$ Extensive research has been done in the area of cube view selection, i.e. deciding which views to materialize $[63,5,57,68]$. The subcategory hierarchy of a compliant EHM dimension contains all the information required to determine a complete set of pre-computed cube views, i.e. a set of cube views that (if materialized) is sufficient for deriving query results at any level. There could exist multiple such sets of views. We can combine results and methods in the literature with the additional information provided by subcategories to better address the problem of cube view selection and materialization. 
$\subseteq$ A canonical mapping in the EHM repair process may produce multiple valid options that differ in the organization of subcategories. Our FIND-PCM algorithm non-deterministically chooses one of the options. The data warehouse users can be involved in the decision making so that a deterministic choice can be made.

$\subseteq$ Our relational schema extension for implementing ROLAP schemas can be formalized with the notion of schema evolution [26]. This can be done by providing a language of schema modification operators to concisely express schema changes. As a result the DBA will be allowed to evaluate the effects of such schema changes. 


\section{Bibliography}

[1] S. Abiteboul, R. Hull and V. Vianu. Foundations of Databases. Addison-Wesley, 1995.

[2] F. Afrati and R. Chirkova. Selecting and using views to compute aggregate queries. Journal Computer System Sciences, 77(6), 2011, pp. 1079-1107.

[3] R. Agrawal, A. Gupta and S. Sarawagi. Modeling Multidimensional Databases. Proc. 13th International Conference on Data Engineering, 1997, pp. 232-243.

[4] M. Alviano, G. Greco and N. Leone. Dynamic Magic Sets for Programs with Monotone Recursive Aggregates. LPNMR 2011, pp. 148-160.

[5] K. Aouiche, P. Jouve and J. Darmont. Clustering-Based Materialized View Selection in Data Warehouses. Advances in Databases and Information Systems 2006, pp. 8195.

[6] A. Arasu, R. Kaushik and J. Li. Data Generation Using Declarative Constraints. SIGMOD, 2011, pp. 685-696.

[7] M. Arenas, L. Bertossi and J. Chomicki. Consistent Query Answers in Inconsistent Databases. Proc. ACM PODS'99, 1999, pp. 68-79.

[8] M. Arenas, L. Bertossi, J. Chomicki, X. He, V. Raghavan and J. Spinrad. Scalar Aggregation in Inconsistent Databases. Theoretical Computer Science, 2003, 296(3), pp. 405-434.

[9] S. Ariyan and L. Bertossi. Structural Repairs of Multidimensional Databases. Proc. Alberto Mendelzon International WS of Foundations of Data Management (AMW'11), CEUR Workshop Proceedings, Vol. 749, 2011.

[10] E. Baralis, S. Paraboschi, and E. Teniente. Materialized Views Selection in a Multidimensional Database. Proc. 23rd International Conference on Very Large Data Bases, 1997, pp. 156-165.

[11] L. Bertossi. Consistent Query Answering in Databases. ACM SIGMOD Record, June 2006, 35(2):68-76.

[12] L. Bertossi. Database Repairing and Consistent Query Answering, Morgan \& Claypool, Synthesis Lectures on Data Management, 2011.

[13] L. Bertossi, L. Bravo and M. Caniupan. Consistent Query Answering in Data Warehouses. In Proc. Alberto Mendelzon International Workshop on Foundations of Data Management (AMW'09), CEUR Workshop Proceedings, Vol. 450, 2009. 
[14] C. Binnig, D. Kossmann, E. Lo, and M. Tamer zsu. QAGen: Generating Query-Aware Test Databases. In SIGMOD Conference, 2007, pp. 341-352.

[15] L. Bravo, M. Caniupan and C. Hurtado. Logic Programs for Repairing Inconsistent Dimensions in Data Warehouses. Proc. of the 4th Alberto Mendelzon International Workshop on Foundations of Data Management (AMW'10), CEUR Workshop Proceedings, Vol. 619, 2010.

[16] N. Bruno and S. Chaudhuri. Flexible database generators. Proc. 31st International Conference on Very Large Data Bases, 2005, pp. 10971107.

[17] L. Cabibbo and R. Torlone. Querying Multidimensional Databases. Proc. DBLP'97, Springer LNCS 1369, 1997, pp. 319-335.

[18] L. Cabibbo and R. Torlone. A Logical Approach to Multidimensional Databases. Advances in Databases Technology (EDTB 1998), LNCS, volume 1337, 1998, pp. 183-197.

[19] M. Caniupan. Handling Inconsistencies in Data Warehouses. In Current Trends in Database Technology, Springer LNCS 3268, 2004, pp. 160-176.

[20] M. Caniupan, L. Bravo, and C. Hurtado. Repairing Inconsistent Dimensions in Data Warehouses. Data \& Knowledge Engineering, 2012, 79(80), pp. 17-39.

[21] M. Caniupan and A. Vaisman. Repairing Dimension Hierarchies under Inconsistent Reclassification. In Advances in Conceptual Modeling. Recent Developments and New Directions, ER Workshops FP-UML, MoRE-BI, Onto-CoM, SeCoGIS, Variability@ER, WISM, Brussels, Belgium, 2011,pp. 75-85.

[22] D. Cheung, B. Zhou, B. Kao, H. Lu, T. Lam and H.F. Ting. Requirement-Based Data Cube Schema Design. In Proc. CIKM'99, 1999, pp. 162-169.

[23] E. Codd. Relational completeness of database sublanguage. In Database Systems (Rustin, Ed.), Prentice Hall, 1972, pp. 65-98.

[24] E. Codd, S. Codd and C. Salley. Providing OLAP (On-line Analytical Processing) to user analysis: An IT mandate. White Paper, Arbor Software, 1993.

[25] S. Cohen, W. Nutt and A. Serebrenik. Rewriting Aggregate Queries Using Views. PODS'99, 1999, pp. 155-166.

[26] C. Curino, H. J. Moon and C. Zaniolo. Graceful Database Schema Evolution: The Prism Workbench. PVLDB, 2008, 1(1):761-772.

[27] A. Datta and H. Thomas. A Conceptual Model and Algebra for On-Line Analytical Processing in Decision Support Databases. Proc. 7th Annual Workshop on Information Technologies and Systems, 1997. 
[28] C. E. Dyreson. Information Retrieval from an Incomplete Data Cube. Proc. 22nd Conference on Very Large Databases, 1996, pp. 532-543.

[29] W. Faber, G. Pfeifer and N. Leone. Semantics and complexity of recursive aggregates in answer set programming. Artificial Intelligence, 2011, 175(1), pp. 278-298.

[30] J. Gray, S. Chaudhuri, A. Bosworth, A. Layman, D. Reichart, M. Venkatrao, F. Pellow, and H. Pirahesh. Data Cube: A Relational Aggregation Operator Generalizing Group-By, Cross-Tab and Sub-Totals. Data Mining and Knowledge Discovery, 1997, $1(1)$, pp. 29-54.

[31] M. Gyssens and L. V. S. Lakshmanan. A Foundation for Multi-Dimensional Databases. Proc. 23rd Conference on Very Large Databases, 1997, pp. 106-115.

[32] J. Han and M. Kamber. Data Mining: Concepts and Techniques. Morgan Kaufmann Publishers Inc., San Francisco, CA, 2000.

[33] V. Harinarayan, A. Rajaraman and J. Ullman. Implementing Data Cubes Efficiently. Proc. SIGMOD, 1996, pp. 205-216.

[34] J. Hoag and C. Thompson. A Parallel General-Purpose Synthetic Data Generator. SIGMOD Record, 2007, 36(1):19-24.

[35] J. Horner and I. Song. A Taxonomy of Inaccurate Summaries and Their Management in OLAP Systems. In Conceptual Modeling - ER 2005, volume 3716 of Lecture Notes in Computer Science, 2005, pp. 433-448.

[36] K. Houkjr, K. Torp and R. Wind. Simple and Realistic Data Generation. Proc. 32nd International Conference on Very Large Databases, VLDB 2006, pp. 12431246.

[37] C. Hurtado. Structurally Heterogeneous OLAP Dimensions. PhD Thesis, Computer Science Department, University of Toronto, 2002.

[38] C. Hurtado and C. Gutierrez. Computing Cube View Dependences in OLAP Datacubes. Proc. International Conference on Scientific and Statistical Database Management, IEEE CS Press, 2003, pp. 33-42.

[39] C. Hurtado and C. Gutierrez. Equivalence of Olap Dimension Schemas. In Data Warehouses and OLAP: Concepts, Architectures and Solutions, Wilhelminenburg Castle, Austria, 2004, pp. 176-195.

[40] C. Hurtado and C. Gutierrez. Handling Structural Heterogeneity in OLAP. In Data Warehouses and OLAP: Concepts, Architectures and Solutions, R. Wrembler and C. Koncilia (eds.), Idea Group, Inc. 2006.

[41] C. Hurtado, C. Gutierrez and A. Mendelzon. Capturing Summarizability With Integrity Constraints in OLAP. ACM Transactions on Database Systems, 2005, 30(3):854-886. 
[42] C. Hurtado and A. Mendelzon. Reasoning about Summarizability in Heterogeneous Multidimensional Schemas. Proc. 8th International Conference on Database Theory, 2001, pp. 375-389.

[43] C. Hurtado and A. Mendelzon. OLAP Dimension Constraints. Proc. PODS'02, 2002, pp. 169-179.

[44] C. Hurtado, A. Mendelzon, and A. Vaisman. Updating OLAP Dimensions. Proc. International Workshop on Data Warehousing and OLAP (DOLAP), ACM Press, 1999, pp.60-66.

[45] C. Hurtado, A. Mendelzon and A. Vaisman. Maintaining Data Cubes under Dimension Updates. Proc. ICDE'99, IEEE CS Press, 1999, pp. 346-355.

[46] W. Inmon. Building the Data Warehouse. 4th Edition, Wiley, 2005.

[47] H. V. Jagadish, L. V. S. Lakshmanan, and D. Srivastava. What Can Hierarchies Do for DataWarehouses? . Proc. 25th International Conference on Very Large Data Bases, 1999, pp. 530-541.

[48] R. Kimball and M. Ross. The Data Warehouse Toolkit: the Complete Guide to Dimensional Modeling. Wiley, 2nd edition, April 2002.

[49] L. V. S. Lakshmanan, A. Russakovsky and V. Sashikanth. What-if OLAP Queries with Changing Dimensions. Proc. ICDE'08, IEEE CS Press, 2008, pp. 1334-1336.

[50] J. Lechtenbrger and G. Vossen. Multidimensional normal forms for data warehouse design. Information Systems, 28(5), 2003, pp. 415-434.

[51] W. Lehner. Modeling Large Scale OLAP Scenarios. Proc. 6th International Conference on Extending Database Technology, 1998, pp. 153-167.

[52] W. Lehner, J. Albrecht, H. Wedekind. Normal Forms for Multidimensional Databases. Proc. 10th International Conference on Scientific and Statistical Database Management, 1998, pp. 63-72.

[53] H. Lenz and A. Shoshani. Summarizability in OLAP and Statistical Data Bases. Proc. International Conference on Scientific and Statistical Database Management, IEEE CS Press, 1997, pp. 132-143.

[54] A. Levy, A. Mendelzon, Y. Sagiv and D. Srivastava. Answering Queries Using Views. Proc. PODS, 1995, pp. 95-104.

[55] L. Libkin. Expressive Power of SQL. Proc. 8th International Conference on Database Theory, 2001, pp. 1-21.

[56] V. Lifschitz. Circumscription. In Handbook of Logic in AI and Logic Programming, Vol. 3, Oxford University Press, 1994, pp. 298-352. 
[57] W. Lin, I. Kuo. A Genetic Selection Algorithm for OLAP Data Cubes. Knowledge \& Information Systems, 2004, 6(1), pp. 83-102.

[58] E. Lo, N. Cheng and W. Hon. Generating Databases for Query Workloads. Proc. 36th International Conference on Very Large Databases, VLDB 2010, pp. 848859.

[59] E. Malinowski, E. Zimnyi. Hierarchies in a Multidimensional Model: From Conceptual Modeling to Logical Representation. Data \& Knowledge Engineering, 2006, 59(2), pp. 348-377.

[60] S. Mansmann, and M. Scholl. Empowering the OLAP Technology to Support Complex Dimension Hierarchies. International Journal of Data Warehousing and Mining, 2007, 3(4), pp. 31-50.

[61] Microsoft Corporation. Multidimensional Expressions (MDX) Reference. Available at http://msdn.microsoft.com/enus/library/ms145506.aspx, 2008.

[62] T. Niemi, M. Niinimaki, P. Thanisch, and J. Nummenmaa. Detecting summarizability in OLAP. Data \& Knowledge Engineering, 2014, Volume 89, pp. 1-20.

[63] T. Palpanas, N. Koudas, and A. Mendelzon. On Space Constrained Set Selection Problems. Data \& Knowledge Engineering, 2008, 67(1), pp. 200-218.

[64] T. B. Pedersen, J. Gu, A. Shoshani and C. S. Jensen. Object-Extended OLAP Querying. Data \& Knowledge Engineering, 2009, 68(5), pp. 453-480.

[65] T. B. Pedersen, C. S. Jensen, and C. E. Dyreson. Extending Practical Pre-Aggregation in On-Line Analytical Processing. Proc. VLDB'99. Morgan Kaufmann, 1999, pp. 663-674.

[66] T. Pedersen, T. Jensen, and C. Dyreson. A Foundation for Capturing and Querying Complex Multidimensional Data. Information Systems (IS), 2001, 26(5), pp. 383423.

[67] T. Rabl, M. Frank, H.M. Sergieh and H. Kosch. A Data Generator for Cloud-Scale Benchmarking. Proc. 2nd TPC technology conference on Performance evaluation, measurement and characterization of complex systems, 2010, pp. 41-56.

[68] A. Shukla, P. Deshpande and J. Naughton. Materialized View Selection for Multi-cube Data Models. Proc. EDBT’00, 2000, pp. 269-284.

[69] G. Spofford, S. Harinath, C. Webb, D.H. Huang, F. Civardi. MDX Solutions: With Microsoft SQL Server Analysis Services 2005 and Hyperion Essbase. Wiley, 2006.

[70] E. Torlak. Scalable test data generation from multidimensional models. Proc. the ACM SIGSOFT 20th International Symposium on the Foundations of Software Engineering, 2012. 
[71] P. Vassiliadis. Modeling Multidimensional Databases, Cubes, and Cube Operations. Proc. 10th International Conference on Statistical and Scientific Database Management, 1998, pp. 53-62.

[72] M. Yaghmaie, L. Bertossi, and S. Ariyan. Repair-Oriented Relational Schemas for Multidimensional Databases. Proc. EDBT'12, 2012, pp. 408-419. 